\title{
EVALUATION OF TRANSMISSION \\ ALTERNATIVES FOR THE RETURN \\ CABLE BAND
}

\author{
by \\ Serge Daigle, B. Eng.
}

A thesis submitted to the Faculty of Graduate Studies and Research in partial fulfillment of the requirements

for the degree of

Master of Engineering

Ottawa-Carleton Institute for Electrical Engineering

Department of Systems and Computer Engineering

Carleton University

Ottawa, Ontario, Canada

September 19, 1997

(C) copyright

1997, S. Daigle 
National Library of Canada

Acquisitions and Bibliographic Services

395 Wellington Street Ottawa ON K1A ON4 Canada
Bibliothèque nationale du Canada

Acquisitions et services bibliographiques

395, rue Wellington Ottawa ON K1A ON4 Canada

Your file Votre reftorence

The author has granted a nonexclusive licence allowing the National Library of Canada to reproduce, loan, distribute or sell copies of this thesis in microform, paper or electronic formats.

The author retains ownership of the copyright in this thesis. Neither the thesis nor substantial extracts from it may be printed or otherwise reproduced without the author's permission.
L'auteur a accordé une licence non exclusive permettant à la Bibliothèque nationale du Canada de reproduire, prêter, distribuer ou vendre des copies de cette thèse sous la forme de microfiche/film, de reproduction sur papier ou sur format électronique.

L'auteur conserve la propriété du droit $d$ 'auteur qui protège cette thèse. $\mathrm{Ni}$ la thèse ni des extraits substantiels de celle-ci ne doivent être imprimés ou autrement reproduits sans son autorisation. 


\begin{abstract}
Our research concerns comparing the performance of various transmission and reception alternatives for the $5-45 \mathrm{MHz}$ portion of the upstream path of the return coaxial cable band ${ }^{1}$. This evaluation is based on ingress noise spectra files collected over an extended period of time from various sites. This data has been used to evaluate return band capacity as a function of sub-channel bandwidth in FDMA and TDMA/FDMA schemes. In addition, the time varying aspect of the channel is considered using variable bit rate allocation to sub-bands, as in OFDM with frequency division multiplexing. Furthermore, the same analysis is repeated for a wideband system using a Decision Feedback Equalizer (WDFE) contending with ingress noise instead of channel distortion. This research demonstrates that using a variable channel allocation (VCA) scheme to match the time varying aspect of the channel enhances the performance over a fixed channel allocation (FCA) scheme. It also shows that a wideband system using an adaptive Decision Feedback Equalizer (DFE) performs similar to a Multi-Carrier Modulation (MCM) method, and that reasonable performance can be obtained by using a system of lower complexity consisting of the combination of the FCA with the WDFE.
\end{abstract}

\footnotetext{
${ }^{1}$ Research sponsored by the Canadian Cable Labs.
} 


\section{Acknowledgements}

I would like to thank my thesis supervisor, Dr. David D. Falconer, for his ideas as well as his tremendous support and guidance throughout this research. I would also like to thank Albert Kim and his colleagues of Rogers Cablesystems Ltd. for their advice and help with the project.

In addition, I would like to express my appreciation to the Canadian Cable Labs for their financial support.

Finally, I would like to express my gratitude to Catherine Reid for her help and support throughout the writing of this thesis. 


\section{Contents}

1 Introduction 1

1.1 Problem Description $\ldots \ldots \ldots \ldots \ldots \ldots$

1.2 Proposed approach $\ldots \ldots \ldots \ldots \ldots \ldots \ldots \ldots$

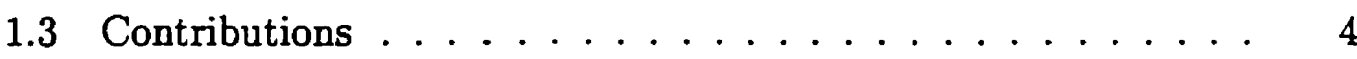

1.4 Thesis structure $\ldots \ldots \ldots \ldots \ldots \ldots \ldots \ldots$

2 CATV systems overview 6

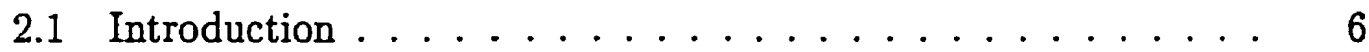

2.2 Network Architecture and Functionality $\ldots \ldots \ldots \ldots$

2.2.1 CATV spectrum $\ldots \ldots \ldots \ldots \ldots$

2.2.2 The MAC level $\ldots \ldots \ldots \ldots \ldots \ldots$

2.3 Upstream channel characterization $\ldots \ldots \ldots \ldots$

2.3.1 Ingress noise spectra files . . . . . . . . . . . 11

2.3.2 Reflections in CATV networks . . . . . . . . . 13

3 Research models $\quad 16$ 


\section{CONTENTS}

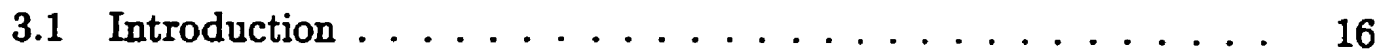

3.2 MCM model . . . . . . . . . . . . . . . 17

3.2.1 MCM characteristics . . . . . . . . . . . . 18

3.2.2 FCA and VCA schemes $\ldots \ldots \ldots \ldots \ldots . \ldots 21$

3.2.3 The modulation schemes . . . . . . . . . . . 23

3.2.4 Evaluation of spectral efficiency . . . . . . . . . 24

3.2.4.1 Ideal channel model . . . . . . . . . . . . 24

3.2.4.2 Distorted channel model . . . . . . . . . 27

3.3 Wideband infinite DFE (WDFE) model . . . . . . . 29

3.3.1 Evaluation of spectral efficiency . . . . . . . . 30

3.4 Wideband Finite DFE (WFDFE) model $\ldots \ldots \ldots \ldots$

3.4.1 DFE characteristics . . . . . . . . . . . . . 31

3.4.2 Finite DFE evaluation details $\ldots \ldots \ldots \ldots \ldots$

3.4.2.1 The additive noise covariance . . . . . . 34

3.4.2.2 Forward autocorrelation matrix and adjacent channel interference . . . . . . . . . 34

3.4.2.3 Capacity with excess bandwidth and guardbands 35

3.4.3 Evaluation of spectral efficiency . . . . . . . . 37

4 Results and analysis 38

4.1 Introduction . . . . . . . . . . . . . . 38

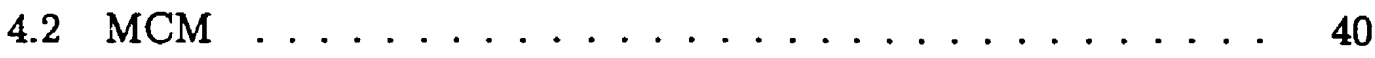

4.2.1 Effect of the sub-band width on capacity . . . . . . 40 
4.2.1.1 An example of bit rate allocation . . . . . . 44

4.2.1.2 5-35 MHz portion . . . . . . . . . . 41

4.2.1.3 5-15 $\mathrm{MHz}$ portion . . . . . . . . . . 44

4.2.1.4 $15-35 \mathrm{MHz}$ portion . . . . . . . . 45

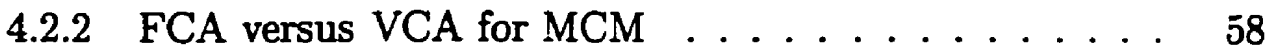

4.2.3 Channel distortion by reflections $\ldots \ldots \ldots \ldots$

4.3 Wideband system with infinite DFE (WDFE) . . . . . 61

4.3.1 Effect of the sub-band width on capacity . . . . . . 61

4.3.1.1 5-35 $\mathrm{MHz}$ portion . . . . . . . . . . 62

4.3.1.2 $5-15 \mathrm{MHz}$ portion . . . . . . . . . 62

4.3.1.3 $15-35 \mathrm{MHz}$ portion . . . . . . . . . 67

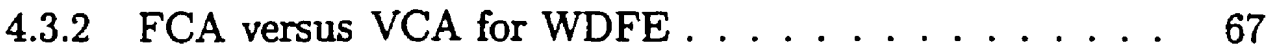

4.3.3 Channel distortion by reflections $\ldots \ldots \ldots \ldots 77$

$4.4 \mathrm{MCM}$ versus WDFE $\ldots \ldots \ldots \ldots \ldots \ldots \ldots$

4.4.1 WDFE using FCA versus MCM with VCA method . . 79

4.5 Wideband system with finite DFE (WFDFE) . . . . . 81

4.5.1 Number of taps . . . . . . . . . . . . . . . 81

4.5.2 The effect of the excess bandwith parameter . . . . 83

4.5.3 Effect of guardbands in adjacent channel interference - 87

4.5.4 Fractionally spaced equalizers . . . . . . . . . . 93

4.5.5 Performance of WFDFE and other models . . . . . 99

5 Conclusions and recommendations 105 


\section{CONTENTS}

5.1 Future research .................. 106 


\section{List of Figures}

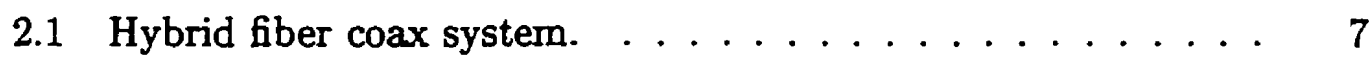

2.2 Frequency allocation plan of a $750 \mathrm{MHz}$ systern. . . . . . 9

2.3 MLAP protocol philosophy. . . . . . . . . . . . 10

2.4 Ingress noise spectra sample of site 1 . Note the presence of the T1/Datacom tester carrier and cable modem carrier located at $25 \mathrm{MHz}$ and $34 \mathrm{MHz}$ respectively. . . . . . . . . . 13

2.5 Ingress noise spectra sample of site 2. Note the presence of the T1/Datacom tester carrier located at $25 \mathrm{MHz} \ldots \ldots \ldots . . .14$

2.6 The top graph shows the equivalent impulse response of a 2.1 $\mathrm{MHz}$ sub-band with 1 reflection delayed by $500 \mathrm{nsec}$ and -20 $\mathrm{db}$ down, and filtered by a raised cosine filter with $25 \%$ excess bandwidth. The bottom graph shows equivalent magnitude response of a CATV channel in the 30-35 $\mathrm{MHz}$ region. . . . . . 15

3.1 MCM scheme model used for the analysis. . . . . . . . 17 


\section{LIST OF FIGURES}

3.2 a) MCM power spectra. b) Remapping of the sub-bands in frequency selective channels. $\ldots \ldots \ldots \ldots$

3.3 Addition of a time guard band in between symbols for the MCM system. . . . . . . . . . . . . . .

3.4 An example of varying (remapping) the modulation scheme to compensate for long time narrow band ingress noise. The first carrier at time $\mathrm{T}+1$ minute has reduced its modulation scheme to compensate for the high noise level now present in the band.

3.5 Constellations used in our analysis. . . . . . . . . . . 23

3.6 Noise averaging results for $2 \mathrm{MHz}$ wide spectra. . . . . . . 25

3.7 Example showing the procedure for evaluating the efficiency using FCA, VCA and VCA-AVG schemes. . . . . . . 26

3.8 Efficiency obtained when conducting an analysis with a $2.1 \mathrm{MHz}$ FDMA-VCA scheme for site 2. The top graph shows the effciency obtained on a 10 minute basis. The bottom graph gives an histogram using the same parameters. . . . . . . . 28

3.9 Infinite taps DFE model used for analysis. . . . . . . . 29

3.10 Wideband system with finite DFE model. . . . . . . . . 31

3.11 Finite DFE equalizer. . . . . . . . . . . . . . . 32

3.12 Equivalent spectra for 3 sub-bands with $50 \%$ excess bandwidth and $1 / \mathrm{T}$ carrier spacing. . . . . . . . . . 36 


\section{LIST OF FIGURES}

4.1 Bit rate allocation for the 5-35 $\mathrm{MHz}$ spectrum of site 1 versus frequency for a FCA scheme. . . . . . . . . . . . . . 42

4.2 Bit rate allocation for the $5-35 \mathrm{MHz}$ spectrum of site 2 versus frequency for a FCA scheme. . . . . . . . . . . . 43

4.3 Efficiency for the 5-35 $\mathrm{MHz}$ spectrum of site 1 versus subchannel bandwidth for a FCA scheme. . . . . . . . . . . 46

4.4 Efficiency for the $5-35 \mathrm{MHz}$ spectrum of site 2 versus subchannel bandwidth for a FCA scheme. . . . . . . . . . . 47

4.5 Efficiency for the $5-35 \mathrm{MHz}$ spectrum of site 1 versus subchannel bandwidth for a VCA scheme. . . . . . . . . . . 48

4.6 Efficiency for the $5-35 \mathrm{MHz}$ spectrum of site 2 versus subchannel bandwidth for a VCA scheme. . . . . . . . . . . 49

4.7 Efficiency for the 5-15 $\mathrm{MHz}$ spectrum of site 1 versus subchannel bandwidth for a FCA scheme. . . . . . . . . . 50

4.8 Efficiency for the 5-15 $\mathrm{MHz}$ spectrum of site 2 versus subchannel bandwidth for a FCA scheme. . . . . . . . . . 51

4.9 Efficiency for the 5-15 $\mathrm{MHz}$ spectrum of site 1 versus subchannel bandwidth for a VCA scheme. . . . . . . . . 52

4.10 Efficiency for the 5-15 $\mathrm{MHz}$ spectrum of site 2 versus subchannel bandwidth for a VCA scheme. . . . . . . . . 53

4.11 Efficiency for the $15-35 \mathrm{MHz}$ spectrum of site 1 versus subchannel bandwidth for a FCA scheme. . . . . . . . . . . . 54 


\section{LIST OF FIGURES}

4.12 Efficiency for the $15-35 \mathrm{MHz}$ spectrum of site 2 versus subchannel bandwidth for a FCA scheme. . . . . . . . . 55

4.13 Efficiency for the $15-35 \mathrm{MHz}$ spectrum of site 1 versus subchannel bandwidth for a VCA scheme. . . . . . . . . . 56

4.14 Efficiency for the $15-35 \mathrm{MHz}$ spectrum of site 2 versus subchannel bandwidth for a VCA scheme. . . . . . . . . . 57

4.15 Performance of a channel with distortion and a channel without distortion for site 2 using the MCM system. . . . . . . . 60

4.16 Efficiency of the 5-35 $\mathrm{MHz}$ spectrum of site 1 versus sub-channel bandwidth for a FCA scheme. . . . . . . . . . . 63

4.17 Efficiency of the 5-35 $\mathrm{MHz}$ spectrum of site 2 versus sub-channel bandwidth for a FCA scheme. . . . . . . . . . 64

4.18 Efficiency for the 5-35 $\mathrm{MHz}$ spectrum of site 1 versus subchannel bandwidth for a VCA scheme. . . . . . . . . . 65

4.19 Efficiency of the 5-35 $\mathrm{MHz}$ spectrum of site 2 versus sub-channel bandwidth for a VCA scheme. . . . . . . . . . . 66

4.20 Efficiency of the $5-15 \mathrm{MHz}$ spectrum of site 1 versus sub-channel bandwidth for a FCA scheme. . . . . . . . . . 68

4.21 Efficiency of the 5-15 $\mathrm{MHz}$ spectrum of site 2 versus sub-channel bandwidth for a FCA scheme. . . . . . . . . . 69

4.22 Efficiency of the 5-15 $\mathrm{MHz}$ spectrum of site 1 versus sub-channel bandwidth for a VCA scheme. . . . . . . . . . . . 70 


\section{LIST OF FIGURES}

4.23 Efficiency of the 5-15 MHz spectrum of site 2 versus sub-channel bandwidth for a VCA scheme. . . . . . . . . . 71

4.24 Efficiency of the $15-35 \mathrm{MHz}$ spectrum of site 1 versus subchannel bandwidth for a FCA scheme. . . . . . . . . .

4.25 Efficiency of the $15-35 \mathrm{MHz}$ spectrum of site 2 versus subchannel bandwidth for a FCA scheme. . . . . . . . 73

4.26 Efficiency of the $15-35 \mathrm{MHz}$ spectrum of site 1 versus subchannel bandwidth for a VCA scheme. . . . . . . . . . 74

4.27 Efficiency of the $15-35 \mathrm{MHz}$ spectrum of site 2 versus subchannel bandwidth for a VCA scheme. . . . . . . . . 75

4.28 Performance of a channel with distortion and a channel without distortion for site 2 using the WDFE with an infinite number

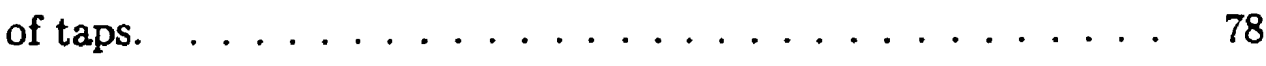

4.29 Taps requirements (2.1 $\mathrm{MHz}$ sub-bands, site 2$). \ldots \ldots \ldots$

4.30 Taps requirements (10.1 $\mathrm{MHz}$ sub-bands, site 2$). \ldots \ldots$

4.31 System efficiency for the low portion of the spectrum (5-15 $\mathrm{MHz}$ ) versus overlapping factor for an equivalent raised cosine filter with $25 \%$ excess bandwidth and $2.1 \mathrm{MHz}$ sub-bands. . . 89

4.32 System efficiency for the low portion of the spectrum (5-15 $\mathrm{MHz}$ ) versus overlapping factor for an equivalent raised cosine filter with $10 \%$ excess bandwidth and $2.1 \mathrm{MHz}$ sub-bands. . 
4.33 System efficiency for the high portion of the spectrum (15-35 $\mathrm{MHz}$ ) versus overlapping factor for an equivalent raised cosine filter with $25 \%$ excess bandwidth and $2.1 \mathrm{MHz}$ sub-bands. . . 91

4.34 System efficiency for the high portion of the spectrum (15-35 $\mathrm{MHz}$ ) versus overlapping factor for an equivalent raised cosine filter with $10 \%$ excess bandwidth and $2.1 \mathrm{MHz}$ sub-bands. . . 92

4.35 System efficiency for the low portion of the spectrum (5-15 $\mathrm{MHz}$ ) versus overlapping factor for an equivalent raised cosine filter with $25 \%$ excess bandwidth and $2.1 \mathrm{MHz}$ sub-bands. . . 95

4.36 System efficiency for the low portion of the spectrum (5-15 $\mathrm{MHz}$ ) versus overlapping factor for an equivalent raised cosine filter with $10 \%$ excess bandwidth and $2.1 \mathrm{MHz}$ sub-bands. . . 96

4.37 System efficiency for the high portion of the spectrum (15-35 $\mathrm{MHz}$ ) versus overlapping factor for an equivalent raised cosine filter with $25 \%$ excess bandwidth and $2.1 \mathrm{MHz}$ sub-bands. .

4.38 System efficiency for the high portion of the spectrum (15-35 $\mathrm{MHz}$ ) versus overlapping factor for an equivalent raised cosine filter with $10 \%$ excess bandwidth and $2.1 \mathrm{MHz}$ sub-bands. . 


\section{List of Tables}

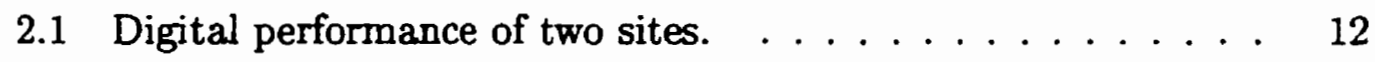

3.1 SNR required to meet the condition for a BER $<10^{-6}$ and $10^{-9}$ for the modulation schemes used in our evaluation. The corresponding spectral efficiency value is given for filters with minimum excess banwidth $\ldots \ldots \ldots \ldots \ldots \ldots$

4.1 Performance of VCA over FCA with MCM scheme with 100 kHz sub-bands. . . . . . . . . . . . . . 59

4.2 Performance of VCA over FCA. . . . . . . . 76

4.3 Fixed channel allocation. . . . . . . . . . . . . . . 79

4.4 Variable channel allocation. . . . . . . . . . . . 79

4.5 Improvement in efficiency of the MCM - VCA over the WDFE - FCA for $2 \mathrm{MHz}$ sub-bands. . . . . . . . . . . . 80

4.6 Average efficiency (VCA - AVG) as defined in section 3.2.4 versus excess bandwidth for the low and high portions of the spectrum with $2.1 \mathrm{MHz}$ sub-bands and synchronous equalization. . 86 
LIST OF TABLES

4.7 Channel capacity in Mbps for the low (5-15 MHz) portion of the spectrum for site $1 \ldots \ldots \ldots$. . . . . . . . . 101

4.8 Channel capacity in Mbps for the high (15-35 MHz) portion of the spectrum for site $1 \ldots \ldots \ldots$. . . . . . . . . . 101

4.9 Channel capacity in Mbps for the low (5-15 MHz) portion of the spectrum for site $2 \ldots \ldots \ldots \ldots$. . . . . . . . 102

4.10 Channel capacity in Mbps for the high (15-35 MHz) portion of the spectrum for site $2 \ldots \ldots \ldots \ldots$. . . . . . . . 102 


\section{Chapter 1}

\section{Introduction}

\subsection{Problem Description}

With the present evolution of technology, the aggressive marketing of new digital services and the deregulation of the telecommunication industry, cable companies must find a way to stay competitive by exploiting and enhancing their cable plants and giving their customers access to these new broadband interactive telecommunication services. In the past, the Community Antenna Television (CATV) architecture was primarily a broadcast network, and the frequency band from $5-45 \mathrm{MHZ}$ was used mainly for testing and monitoring purposes. This portion of the cable spectrum is now key to the launching of new digital services since it would be able to provide the upstream link required to furnish bi-directionality in most of these services. A major problem is that this portion of the spectrum is severely hampered by a noisy environment: 
human made impulse noise and narrowband interferences, such as short-wave radio stations, make this portion of the spectrum nearly unusable (non-impulse noise of this type is called ingress noise). Another major factor that must be taken into consideration is the noise funneling effect, or reverse noise buildup, due to the tree and branch topology of the CATV networks. The forward buildup is proportional to the log of the number of series connections while the reverse buildup is equal to the log of the total number of connections [1]. In an all coaxial cable network, with the summation noise of 500 amplifiers in reverse, we typically achieve a signal to noise ratio (SNR) of $13 \mathrm{~dB}$ in reverse. (The terms forward and reverse are commonly used to describe the direction of transmission of the signal, where forward means that the signal is transmitted from the head-end to the subscriber and where the reverse term is used for the opposite direction of transmission.) With a $13 \mathrm{~dB}$ SNR, only low efficiency schemes can be used to convey the data to the head-end. This problem can be resolved, however, when cable operators replace their main trunks with fiber optics technology. By extending fiber links closer to subscribers, the operators are able to reduce the node size (a node is where the optical to electrical conversion occurs), thereby providing better services and signal quality while reducing the upstream noise funneling effect. Presently, efforts are being made to identify the various possible sources of impairment such as reflections (network component mismatch) and network leaks, such as bad connectors, taps and shielding. Many tests are also being done to characterize the upstream channel. 
The objective of this research is to find, through comparative evaluation, the best transmission alternatives that could be used in the $5-45 \mathrm{MHz}$ portion of the spectrum while taking into account the ingress noise. Much of the data used in the analysis was provided by Cable Labs, a company located in Boulder, Colorado. Note that high level aspects, such as the one found at the MAC-level, would be implemented on top of this analysis and should be studied in future research.

\subsection{Proposed approach}

Two upstream transmission approaches have been used in this comparative evaluation. The first approach consists of a carrier modulation system (MCM) where the parallel transmission of data is carried out by using very narrow subbands. The second method consists of a wideband system where the serial transmission of data is carried out by using a few large sub-bands and decision feedback equalization (WDFE). Note here that the practical implementation of a MCM system would use sub-bands in the order of $\mathrm{kHz}$ and that the WDFE would use sub-bands in the order of MHz. The MCM system can therefore be viewed as a parallel modulation method while the WDFE can be viewed as a serial modulation method using a few large sub-bands.

For each of these methods, we will evaluate the return band spectral efficiency as a function of sub-channel bandwidth. In addition, the return band spectral efficiency will be evaluated by considering the following: an optimized 
fixed channel allocation scheme (FCA), where the modulation scheme would be fixed in time but would have the best modulation scheme in each of the individual sub-bands chosen on the basis of the long term average SNR in each sub-band; and a variable channel allocation scheme (VCA), where an adaptive modulation scheme is used (a time variable bit rate allocation scheme which responds to time variations in individual sub-band SNRs due, for example, to interference sources beginning and ending). Furthermore, since most of the noise energy is located in the first portion of the spectrum, the return band performance will be evaluated by considering the 5-15, 15-35 and 5-35 $\mathrm{MHz}$ portion of the band.

In the case of the wideband system, our research also took into consideration an actual system encountered in practice with the examination of how fixed transmitting filters, fixed receiving filters, guard bands, adjacent channel interference, and the number of taps affect return band spectral efficiency.

\subsection{Contributions}

Through this research, it will first be shown that the spectral efficiency of a MCM system can be improved when narrow width sub-bands are used. Second, it will be demonstrated that spectral efficiency is enhanced when a MCM-VCA scheme is used. Third, it will be shown that a wideband system using a DFE can have comparable or superior performance over a MCM system when using a FCA scheme, and that both the MCM and the WDFE present 
similar results when used with a VCA scheme. Finally, for the wideband system implementation, results showing the taps requirements, the effect of the fixed transmitting and receiving filters, and the importance of fractionally spaced equalization in the presence of adjacent channel interference will be given. It will also be shown that we can effectively improve the performance of a wide band system by using carriers spaced in such way that their individual spectra overlap. These investigations are based on actual ingress noise spectra collected from two cable sites.

\subsection{Thesis structure}

The remaining chapters of this thesis are organized as follows: Chapter 2 presents a brief overview of the cable television network and infrastructure, followed by the upstream channel characterization and information on the data provided by Cable Labs; Chapter 3 is dedicated to the model and schemes used to do our comparative evaluation of potential transmission alternatives; and Chapter 4 presents the results and analysis for the MCM, WDFE, and for the practical implementation of a wide band system with finite decision feedback equalization (WFDFE). Chapter 5 summarizes the major conclusions of this study and provides recommendations for future research. 


\section{Chapter 2}

\section{CATV systems overview}

\subsection{Introduction}

This chapter provides an overview of CATV systems, and is divided into two sections. The first section provides a brief review of the network architecture and its components, then presents the spectrum and gives an example of a spectrum allocation plan that could accommodate the new digital services. A general view of how the system could work at the MAC level is also provided. The second section of this chapter has been dedicated to the upstream channel characterization. It provides details on the data that is used for our evaluation and on the reflections found on the upstream channel. 


\subsection{Network Architecture and Functionality}

Figure 2.1 summarizes the HFC network architecture. The network has the form of a tree and branch topology. The system consists basically of a centralized head-end, where all the signal reception and transmission over the network is handled, and a distribution plant, which transports all of the information to the customers. To provide broadband interactive services, a network management infrastructure is implemented at the head-end so that access to the various service platforms such as telephony and the Internet can be controlled. The papers [2], [3] give a good analysis of the requirements and challenges in managing the HFC network.

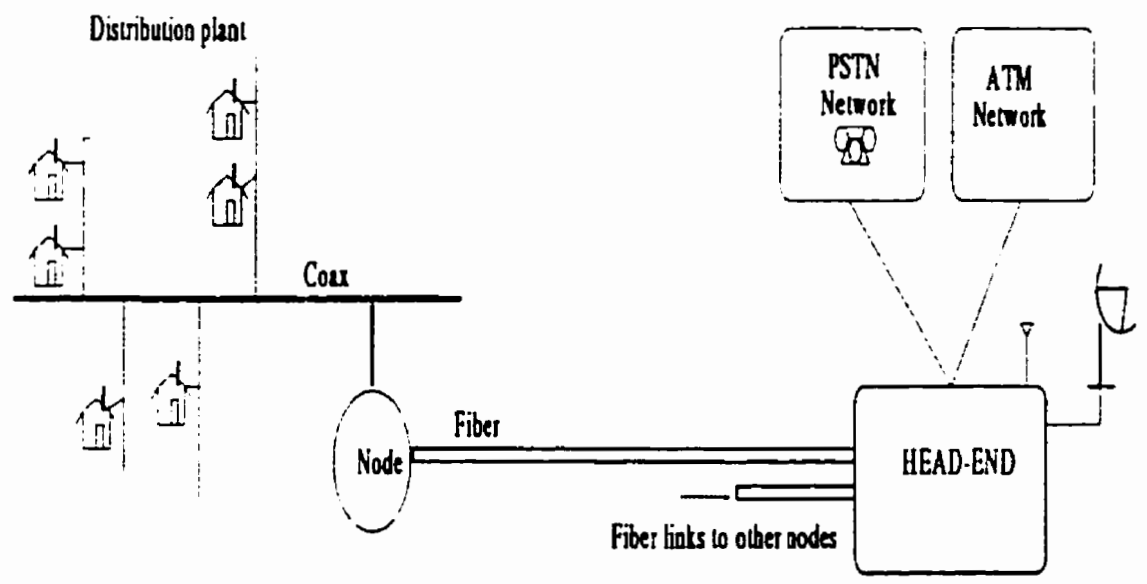

Figure 2.1: Hybrid fiber coax system. 


\subsubsection{CATV spectrum}

Figure 2.2 shows a typical frequency allocation plan [1], [17] that could be used to provide the broadband interactive services for a $750 \mathrm{MHz}$ system. Since most of the present networks provide only up to approximately $700 \mathrm{MHz}$, the new digital services (the downstream portion) could be located at the upper part of the spectrum, while the $50-450 \mathrm{MHz}$ portion could be kept for standard analog channels. The lower part of the spectrum, from $5-45 \mathrm{MHz}$ (the portion under study in this thesis), was used in the past as an upstream link for network management and monitoring purposes and is now proposed to provide the upstream path for the two way requirement of digital services. An interesting fact about the $5-45 \mathrm{MHz}$ spectrum is that this bandwidth could be used as a bi-directional path operating passively; that is, without any amplifiers [4]. The cable loss at these frequencies is low and if the node sizes are small enough, a passive path could be accommodated even during power failures. In section 2.3 , the $5-45 \mathrm{MHz}$ portion of the spectrum is discussed further.

\subsubsection{The MAC level}

Because of the tree and branch topology of the HFC, many subscribers will be sharing an assumed bandwidth. A suitable MAC protocol must therefore be found to provide efficient bandwidth utilization and accommodate the various digital services (telephony, video, data). The IEEE 802.14 committee is presently working on the physical (PHY) and medium access control (MAC) 


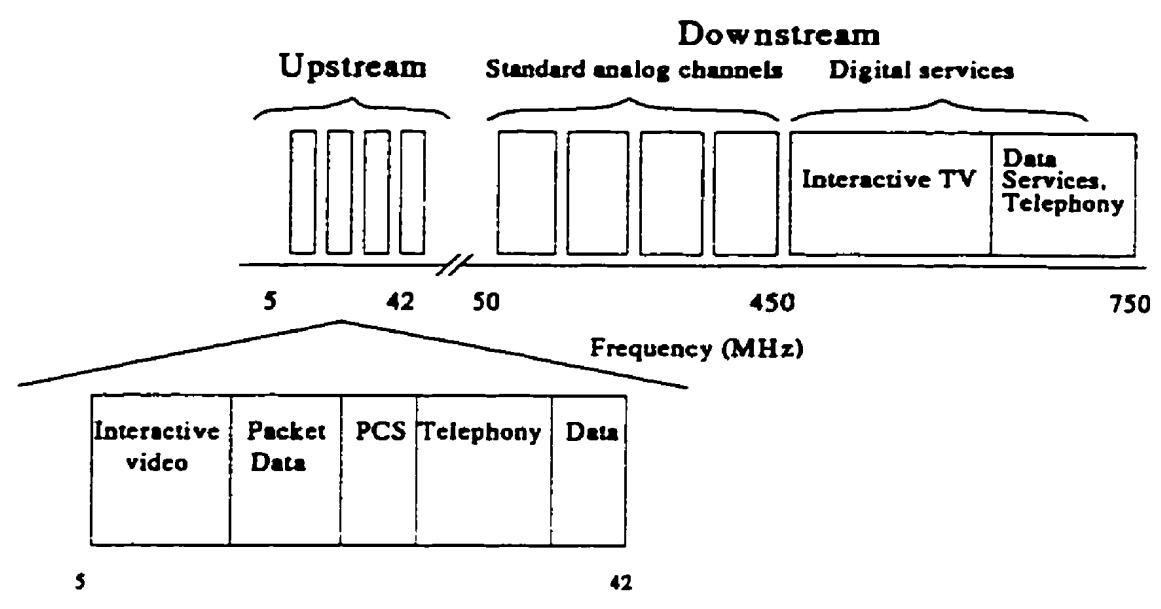

Figure 2.2: Frequency allocation plan of a $750 \mathrm{MHz}$ system.

layers of CATV networks. The papers [5], [6] present a modified version of a combined FDMA/TDMA scheme that could be used at the MAC level. Since our research is more oriented towards the transmission aspect, only a brief description is given of how the MAC processor could work. As an example, the upstream bandwidth of $5-45 \mathrm{MHz}$ could be subdivided in sub-bands with a width of 1-2 MHz. These sub-bands would have their bit stream subdivided in the time domain and would accommodate time frames such as asynchronous transfer mode (ATM) or synchronous transfer mode (STM). Time slots of a particular frame would then be assigned for contention or for signaling purposes. The rest of the frame could be used to convey various types of data to the hecd-end. On the downstream side, the bandwidth reserved for the digital services (probably located above $400 \mathrm{MHz}$ ) would be divided in wider bands such as $6 \mathrm{MHz}$ and would be able to convey more information towards the 
subscriber. Some of these frames would also be responsible for carrying data or MAC protocol information. A bandwidth controller, located at the headend, would be responsible for scheduling transmission on the upstream path. A random access protocol on assigned contention time slot (by the bandwidth controller) would be used to request bandwidth for the subscriber. Figure 2.3, modified from [6], provides a summary of these operations.

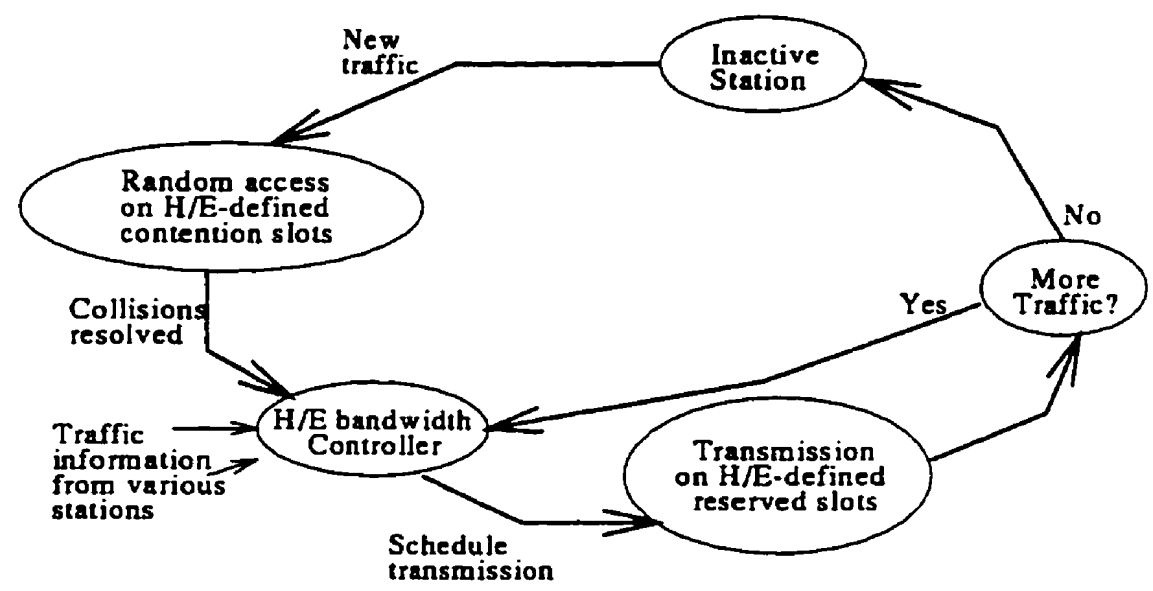

Figure 2.3: MLAP protocol philosophy.

\subsection{Upstream channel characterization}

As mentioned in Chapter 1, many efforts are presently being made to characterize the upstream channel. For this study, previously gathered data provided by Cable Labs has been used in our comparative evaluation. The following section gives a detailed explanation of the files that were used for our analysis. 


\subsubsection{Ingress noise spectra files}

The files were gathered by doing a reverse ingress sweep test to capture the ingress noise level present in the reverse portion of the cable distribution plant. The reverse ingress sweep test consists essentially of sweeping the reverse trunk input from 5-45 $\mathrm{MHz}$ with a spectrum analyzer. On a minute to minute basis, each sample ingress file will show where the spurious energy signals are located at that particular time. Figures 2.4 and 2.5 show two noise ingress spectrum sample files obtained from this test for two distinct CATV network sites. Note that the reverse ingress spectra have been measured with various network configurations. For this research, we have used the all-on configuration (meaning that all the bridger switches were turned on to allow the reverse signal from the feeders to get into the trunk and be transported to the head-end) to consider the maximum noise funneling effect. In site 1 , the measurements are averaged over 34 hours ( 1 minute sample interval). In site 2 , the measurements interval is approximately 60 hours (10 minutes sample interval).

Table 2.1 gives the size and type of the two networks used for our analysis and a summary of the measured digital performance parameters according to the Consultative Committee for International Telegraph and Telephony (CCITT) G.821. The digital performance was measured with a T1/Datacom tester during the same period and the T1 carriers were located at 25 and 23 $\mathrm{MHz}$ for site 1 and 2 respectively. The \%ES, \%SES, \%AVAIL are evaluated according to the CCITT G.821 definition, where \%ES is the percentage of 
Table 2.1: Digital performance of two sites.

\begin{tabular}{|c|c|c|}
\hline Parameter & Site 1 & Site 2 \\
\hline \hline Subscribers & 5500 & 8000 \\
\hline Type & HFC & Coax \\
\hline BER & $1.81 \mathrm{E}-06$ & $20 \mathrm{E}-05$ \\
\hline \%ES & 9.661 & 10.18 \\
\hline \%SES & 0.063 & 0.078 \\
\hline \%AVAIL & $100 \%$ & $99.98 \%$ \\
\hline
\end{tabular}

one-second intervals in which any bit errors occurred, \%SES is the percentage of one-second intervals to have a BER worse than $10^{-3}$, and \%AVAIL is the percentage of time that the system was available. A period of unavailable time begins when the bit error ratio is worse than $10^{-3}$ for a period of ten consecutive seconds. A new period of available time begins with the first second of a period of ten consecutive seconds, each of which is better than $10^{-3}[18]$. Various signals such as cable modem and monitoring carriers were also present during the measurements and were removed to perform the analysis. Since the measurements for site 1 were from $5-35 \mathrm{MHz}$ compared to $5-45 \mathrm{MHz}$ for site 2, we have done our study in the first $5-35 \mathrm{MHz}$ portion of the spectrum so as to compare the performance between both sites. By looking at figure 2.4 and figure 2.5, we can see that most of the noise is located in the first $5-15 \mathrm{MHz}$ portion of the band. As we will see further in our analysis, that portion of the 
spectrum is nearly unusable when using conventional modulation schemes.

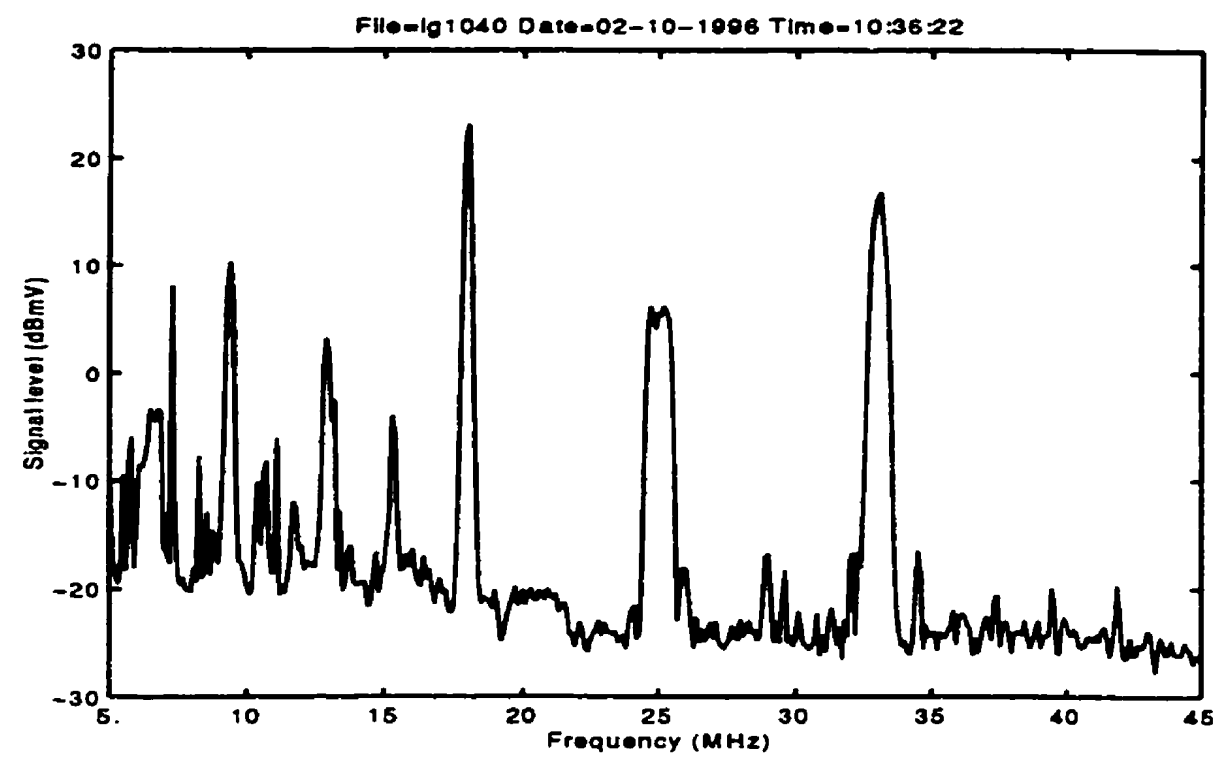

Figure 2.4: Ingress noise spectra sample of site 1. Note the presence of the $\mathrm{T} 1 /$ Datacom tester carrier and cable modem carrier located at $25 \mathrm{MHz}$ and $34 \mathrm{MHz}$ respectively.

\subsubsection{Reflections in CATV networks}

Except for the effect of reflections, the upstream channel of a CATV network has almost ideal frequency response characteristics. The spacing of line amplifiers is determined by the cable attenuation and the loss in the devices inserted in the cable. The levels of the line amplifiers are adjusted to provide cascaded sections with unity gain [9]. The reflections are caused by mismatch in the network and the insertion of taps and splitters. The paper [7] gives the re- 


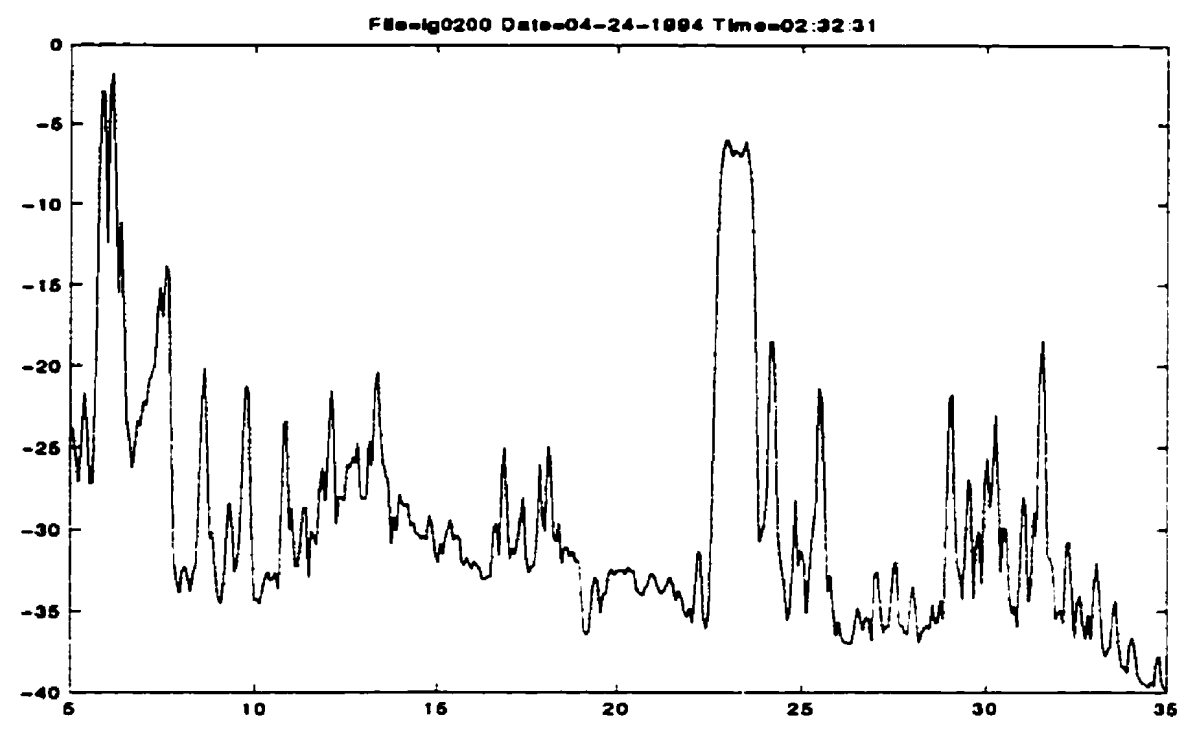

Figure 2.5: Ingress noise spectra sample of site 2. Note the presence of the T1/Datacom tester carrier located at $25 \mathrm{MHz}$.

flection and the delay parameters for a common system. Typically, a value of $-20 \mathrm{~dB}$ down with a delay of 500 nsec seems appropriate to conduct our analysis. Figure 2.6 shows the impulse response and frequency spectrum for the channel using these parameters, with an equivalent excess bandwidth filter of $25 \%$. Notice the ripple effect caused by the reflection on the magnitude of the spectrum. The figure shows the baseband equivalent impulse response for a $2 \mathrm{MHz}$ wide channel. 

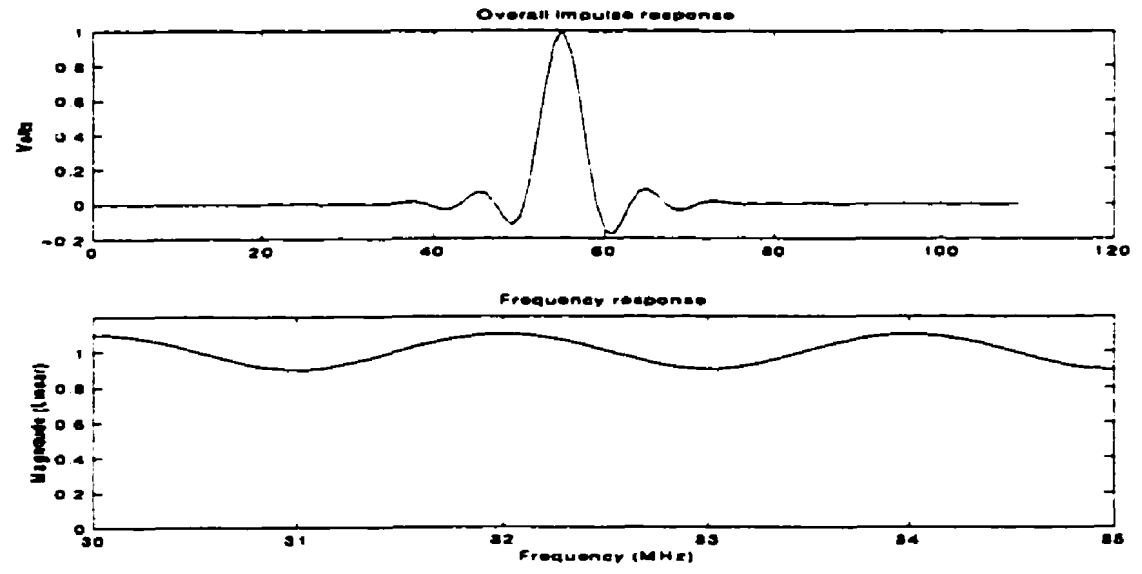

Figure 2.6: The top graph shows the equivalent impulse response of a $2.1 \mathrm{MHz}$ sub-band with 1 reflection delayed by $500 \mathrm{nsec}$ and $-20 \mathrm{db}$ down, and filtered by a raised cosine filter with $25 \%$ excess bandwidth. The bottom graph shows equivalent magnitude response of a CATV channel in the $30-35 \mathrm{MHz}$ region. 


\section{Chapter 3}

\section{Research models}

\subsection{Introduction}

The objective of this chapter is to describe the models that were used for our comparative evaluation. The MCM model will be presented with all the details needed to understand the results and analysis of our evaluation, which are presented in Chapter 4. All the models used in our study consider only the upstream path (the transmission from subscribers toward the head-end). The WDFE and WFDFE will be described without repeating the information common to all the models. 


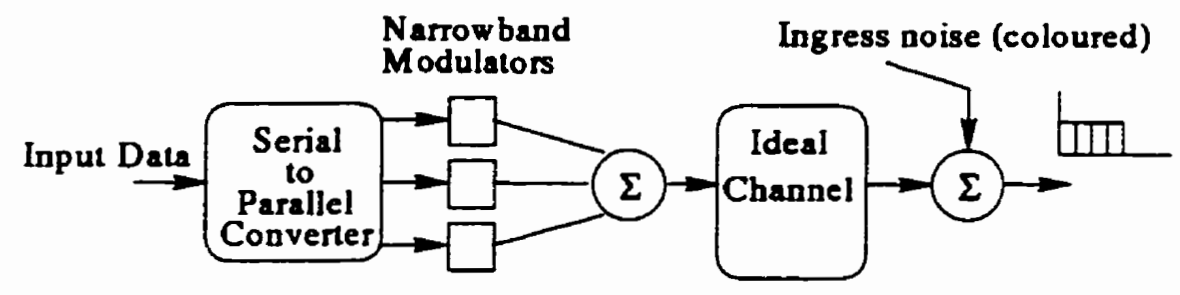

Figure 3.1: MCM scheme model used for the analysis.

\subsection{MCM model}

The first model that was used in our comparative evaluation is the MCM or parallel modulation method (figure 3.1). The following equation is an equivalent representation of the model, where $f_{n}$ would be the corresponding $n^{\text {th }}$ carrier frequency, $a_{k}$ the data symbols and $p(t)$ the Nyquist impulse response.

$$
y(t)=\sum_{k} \sum_{n} a_{n, k} e^{j 2 \pi f_{n} t} p(t-k T)
$$

The MCM system splits the input stream at the data rate $\mathrm{R}$ into $\mathrm{N}$ streams. The data rate of the individual subcarriers becomes $R / N$. The carriers are spaced exactly $\frac{1}{T}$ apart (figure 3.2a), thus $f=\frac{1}{T}$ would correspond to the subband width of the individual sub-carriers. Note that this type of modulation is also used on the asymmetric digital subscriber loop (ADSL), where up to 600 $\mathrm{kb} / \mathrm{s}$ upstream and $1 \mathrm{Mb} / \mathrm{s}$ downstream are transmitted on standard twisted pair. It is also part of the DAB standard in Europe [8] and is presently under study for broadband wireless applications. It has been proposed for upstream Hybrid Fiber-Coax (HFC) system in [10]. 


\subsubsection{MCM characteristics}

By doing parallel modulation of the data, much is gained. First, since the rate of each individual subcarriers is low, the MCM signal is integrated over a long symbol period to recover the information and therefore possesses good immunity to impulse noise. Second, the MCM system is also very good in the presence of frequency selective channels (figure $3.2 \mathrm{~b}$ ), in that the few carriers that are seriously degrarled can be disabled and data can be allocated to other locations (note that since we are using very narrow width sub-bands, the channel will be nearly flat in the individual sub-bands).

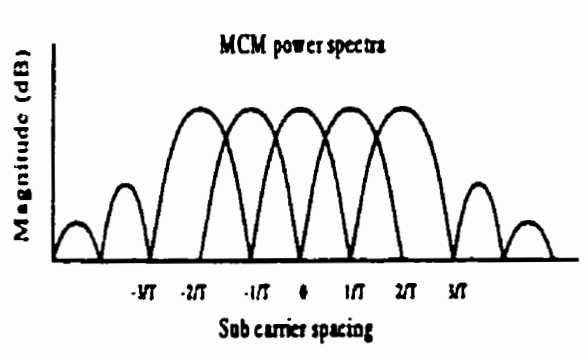

(a)

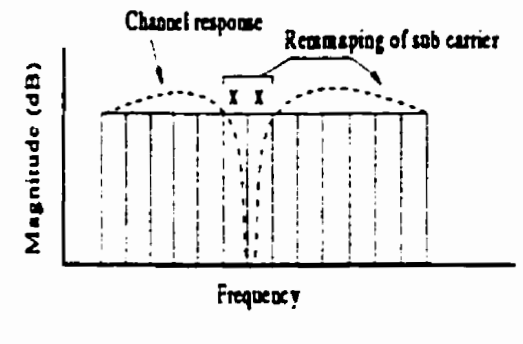

(b)

Figure 3.2: a) MCM power spectra. b) Remapping of the sub-bands in frequency selective channels.

In the CATV environment, the channel is nearly ideal except for a few reflections. The characteristic described above is therefore not really applicable. We can, however, use the remapping capability against slowly varying ingress noise, which has a nonwhite spectrum, by allocating proper bit rate on sub-channels according to their SNR. Another advantage of the MCM is the 
effective bandwidth used for transmission. By using orthogonal carriers, we remove the need for guardbands and thus improve the bandwidth efficiency. Moreover, if we use a large quantity of sub-carriers, the overall spectrum will closely resemble the ideal Nyquist spectrum. An important characteristic that must be preserved in a MCM system is the orthogonality between the signals. MCM systems usually use a cyclic prefix [11] to expand the period $T$ to be able to keep this orthogonality (figure 3.3).

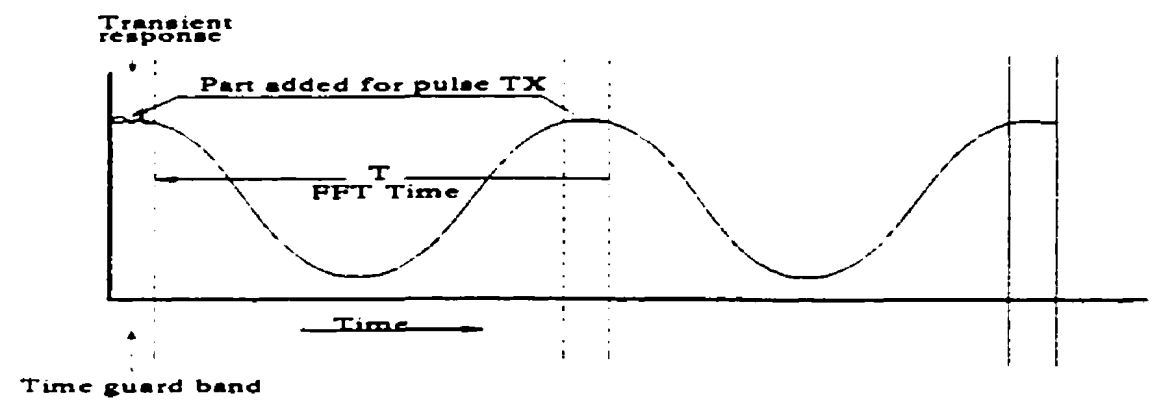

Figure 3.3: Addition of a time guard band in between symbols for the MCM system.

Recall that the output of a linear system consists of a transient part and a steady state part. By expanding or adding a time guard in between symbols long enough to remove the transient part, we are able to keep this orthogonality. The following equation expresses the orthogonality condition at the output of $n^{\text {th }}$ filter. $z_{m}$ represents the complex envelope of the modulated carrier, $\varphi_{m}(t)=e^{j 2 \pi f_{m} t}$ represents the sub-carriers and $H_{m} e^{j \theta_{m}}$ represents the corresponding magnitude and phase response for the $m^{\text {th }}$ carrier. 


$$
U_{n}(t)=R e\left\{\sum_{m=0}^{N-1} z_{m} \int_{0}^{T} H_{m}(t) e^{j \Theta_{m}(t)} \cdot \varphi_{m}(t) \cdot \varphi_{n}(t) d t\right\}
$$

If we wait until the the transient effect has disappeared, the sub-channel $H_{m}(t)$ becomes constant with time and can be removed from the integration. The output of the filter will be zero when $m \neq n$.

$$
U_{n}(t)=R e\left\{\sum_{m=0}^{N-1} z_{m} \cdot H_{m} e^{j \Theta_{m}} \int_{0}^{T} \varphi_{m}(t) \cdot \varphi_{n}(t) d t\right\}
$$

Note that the orthogonality of the MCM carriers can generally be affected only by the parameter $H_{m} e^{j \theta_{m}}$ (channel distortion). This means that impulse noise and additive noise have no effect on the orthogonality condition. Furthermore, if the added cyclic prefix is long enough, the reflections found in the CATV networks will not affect the orthogonality and will take away the need of an equalizer to remove intersymbol interference (ISI). Typically, 99\% of the reflections found in a CATV network are shorter than $1.5 \mathrm{usec}$ [7], [11]. Note also that the sub-carriers become orthogonal only when the symbols are sampled simultaneously on all of the individual subcarriers. Good symbol and carrier synchronization is important throughout the entire MCM system. Special care should be exercised with very narrow sub-bands systems because of the long symbol period.

As mentioned in [12], there is an interesting duality involved with the performance of MCM between the time and frequency domain. The MCM system may be less sensitive to an impulse in the time domain, but would 
be more sensitive to a tone in the frequency domain if the tone interferes with a particular sub-band. As well, since we are using many bit streams to transmit the data, we have less dynamic range for the MCM system. In fact, if all the carriers of the MCM were in phase, a resulting high peak would be obtained and could cause saturation at the transmitter, leading to non-linear distortion and large second and third harmonic spectral rays. Phase jitter is also a problem for $\mathrm{MCM}$ implementation, where side bands are generated that contribute to the noise distortion which can be added to the noise on a power basis [12].

\subsubsection{FCA and VCA schemes}

As was mentioned in Chapter 2 in the MAC level protocol, the receiver (or client) would listen on the downstream portion of the spectrum, located above the $450 \mathrm{MHz}$ portion of the spectrum, for contention slots and would use upstream slots scheduled by the head-end. As a result, it would be able to transmit data on the upstream portion. This protocol could be used with the MCM method using similar FDMA/TDMA techniques with assigned bits at particular times and frequencies.

In our analysis, we studied two optimized bit allocation methods that could be used with the MCM system. The first consisted of an optimized FCA method where the modulation scheme would use a fixed (time-invariant) modulation rate for each individual sub-channel but the rate could vary from 
sub-channel to sub-channel depending on its long term average SNR. The second consisted of a time varying allocation method where the modulation scheme would be able to adapt itself to the slowly varying ingress noise (figure 3.4). The use of a VCA scheme, in the interest of improving system efficiency, would have the drawback of increasing the complexity and overhead of the MAC protocol. Further discussion in regards to the FCA and VCA schemes can be found in section 3.2.4.

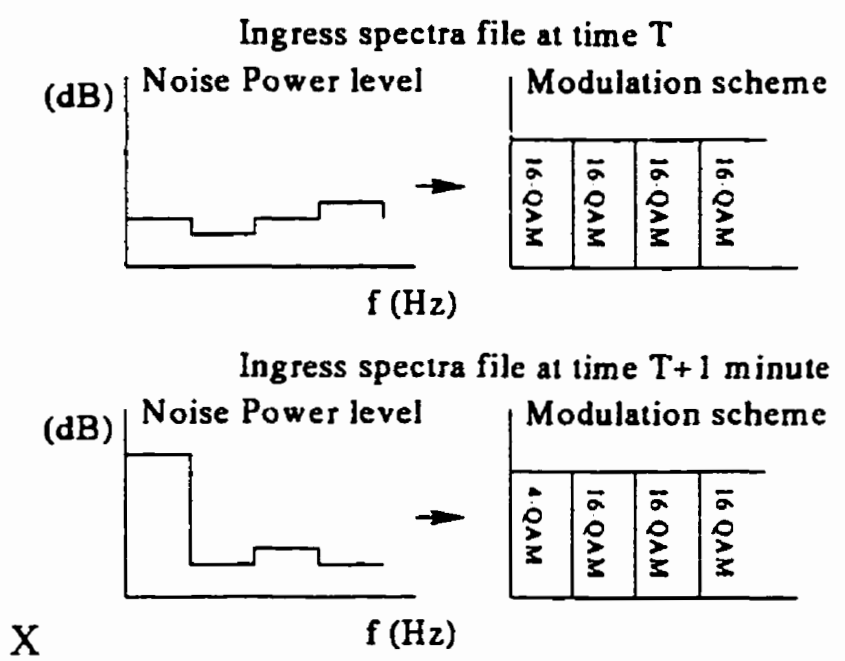

Figure 3.4: An example of varying (remapping) the modulation scheme to compensate for long time narrow band ingress noise. The first carrier at time $\mathrm{T}+1$ minute has reduced its modulation scheme to compensate for the high noise level now present in the band. 


\subsubsection{The modulation schemes}

The modulation schemes used in our analysis are found in the figure and table that follow. Figure 3.5 shows the constellation for each of the schemes, and table 3.1 gives the SNR level required in Additive White Gaussian Noise (AWGN) for the BER of $10^{-6}$ and $10^{-9}[14]$. Note that in our analysis, we assumed no coding of the data.

Table 3.1: SNR required to meet the condition for a BER $<10^{-6}$ and $10^{-9}$ for the modulation schemes used in our evaluation. The corresponding spectral efficiency value is given for filters with minimum excess banwidth

\begin{tabular}{|c|c|c|c|}
\hline Modulation & SNR, $p b<10^{-6}$ & SNR, $p b<10^{-9}$ & Efficiency $(b / s / H z)$ \\
\hline \hline BPSK & 10.5 & 12.5 & 1 \\
\hline QPSK & 13.5 & 15.5 & 2 \\
\hline 8-PSK & 18.7 & 20.8 & 3 \\
\hline 16-QAM & 20.4 & 22.5 & 4 \\
\hline
\end{tabular}
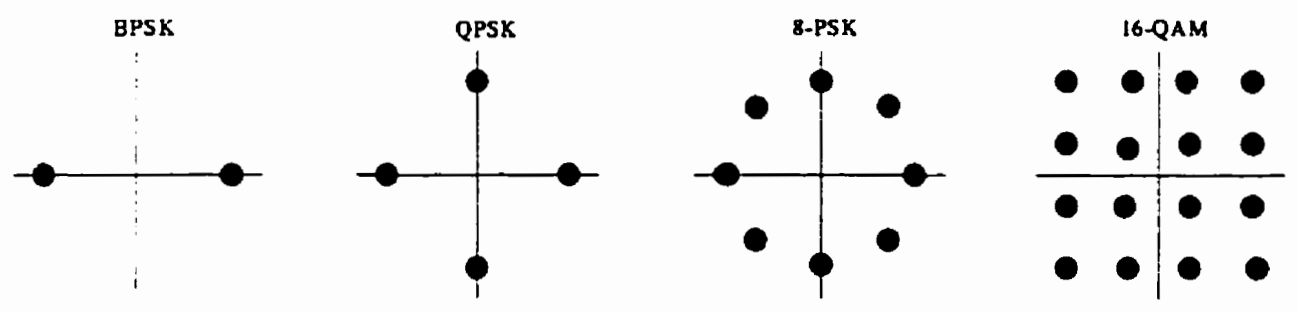

Figure 3.5: Constellations used in our analysis. 


\subsubsection{Evaluation of spectral efficiency}

\subsubsection{Ideal channel model}

We have defined the spectral efficiency as follows: it is the amount of bits per second per hertz that can be effectively transmitted through the portion of the spectrum under study. To compute the spectral efficiency of a MCM model, the total upstream bandwidth from $5-35 \mathrm{MHz}$ was subdivided in sub-bands of width $f_{s b}$ (figure 3.6). The additive noise was assumed to be piecewise constant in each of the sub-bands. The analysis was then conducted on each of the subbands as follows: the average received signal power $P(f)(9 \mathrm{dBmV} / \mathrm{MHz})$ and the noise power $N(f)$ (obtained from the measured ingress noise spectrum with a spectral resolution $\Delta f$ of $100 \mathrm{kHz}$ ) were calculated in order to compute the SNR in each sub-band (equation 3.3).

$$
S N R_{f_{b b}}=\left[\frac{P(f) \cdot f_{s b}}{\sum_{f_{s b}} N(f) \Delta f}\right]
$$

Note that no simulation is involved in the analysis. Once the SNR is found in a particular sub-band, theoretical curves for AWGN noise giving the BER versus SNR for the various modulation schemes are used to make the analysis. SNR values for the BER requirements of $10^{-6}$ and $10^{-9}$ and the corresponding modulation scheme can be found in table 3.1. The spectral efficiency of each sub-band was that of the most efficient modulation scheme (table 3.1) that met the SNR requirements for a BER performance smaller than $10^{-6}\left(10^{-9}\right)$. For a FCA scheme, the selected modulation scheme had to meet these require- 


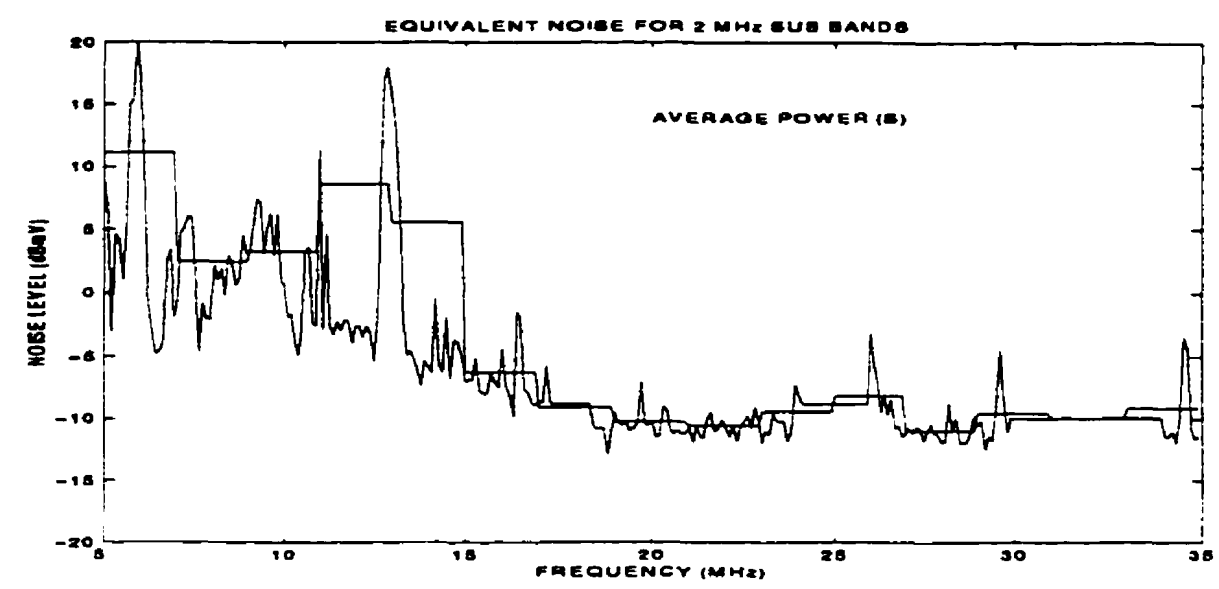

Figure 3.6: Noise averaging results for $2 \mathrm{MHz}$ wide spectra.

ments on a given sub-band for $99 \%$ of the files (1\% outage). A file consists of 400 sample points taken with a spectrum analyzer measuring the ingress noise present in the spectrum. A file therefore represents the noise present in the upstream spectrum at a particular time for a particular site. For the VCA scheme, the modulation scheme could change on a file to file basis. The performance of VCA was found by evaluating the maximum throughput that can be obtained over $99 \%$ of the files ( $1 \%$ outage). The total capacity ${ }^{1}$ of the return bands was then obtained by summing up the spectral efficiency of each sub-band, multiplied by their bandwidths. Note that no power optimization was needed in this approach since it has already been shown that a scheme like MCM optimally distributes the power more or less uniformly over the spec-

${ }^{1}$ Throughout the text, the term capacity should be interpreted as the maximum achievable raw bit rate. 
trum with high SNR [12]. The performance of MCM would therefore not be greatly enhanced by power optimization ${ }^{2}$. This procedure was done considering the 5-15, 15-35 and 5-35 $\mathrm{MHz}$ portions of the spectrum and considering $.1, .2, .5,1,2,5,10 \mathrm{MHz}$ sub-band widths.

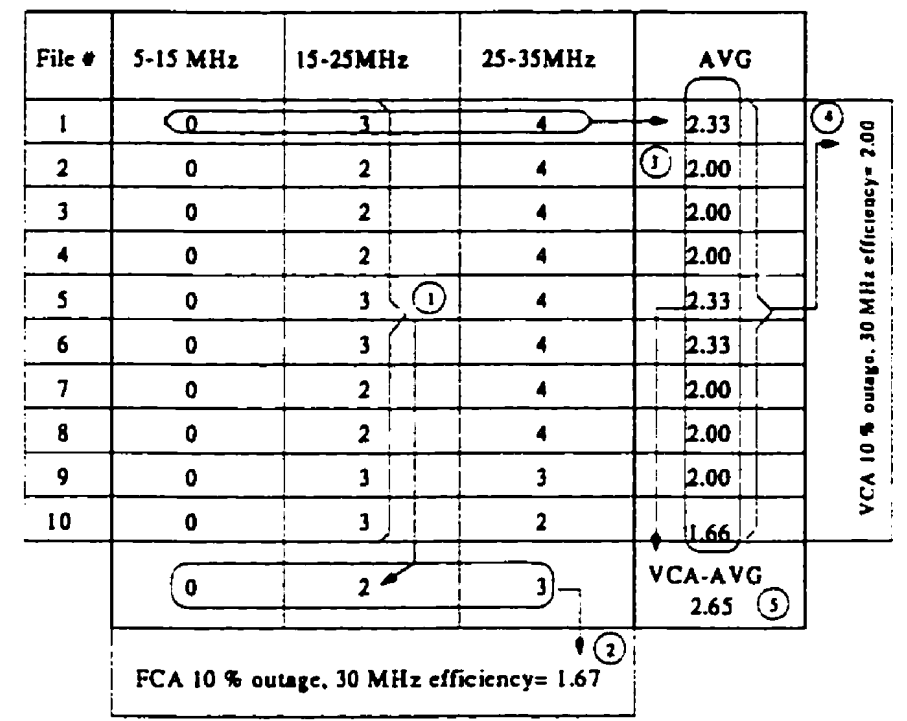

(1) For FCA with $10 \%$ outege, 9 out of 10 files must respect the BER requirements which results in to an efficicncy of $2 \mathrm{~b} / \mathrm{s} / \mathrm{th}$ in that particular sub-band

(2) The wolal spectrum efficiency for FCA is found by averaging the efficiency in each sub-band leading to a value of $1.67 \mathrm{~b} / \mathrm{s} / \mathrm{Hz}$

(3) For VCA we first evaluate the average efriciency oblained for each file

(4) For VCA with $10 \%$ outege, 9 out of 10 files must respect the BER requirements which results in to a toul specturn efficiency of $2 \mathrm{~b} / \mathrm{s} / \mathrm{Hz}$

(5) The VCA-AVG value is found by areraging the efficiency obuined with cach file leading to en efficiency of $2.65 \mathrm{~b} / \mathrm{s} / \mathrm{Hz}$

Figure 3.7: Example showing the procedure for evaluating the efficiency using FCA, VCA and VCA-AVG schemes.

\footnotetext{
${ }^{2} P(f)$ will be normalized to 1 for the remainder of the text
} 
Figure 3.7 illustrates this procedure. The VCA-AVG represent the average throughput that can be obtained from the channel without the outage parameter. Note that for illustrative purposes, only 10 sample ingress files from site 1 were considered with an outage of $10 \%$ in the example.
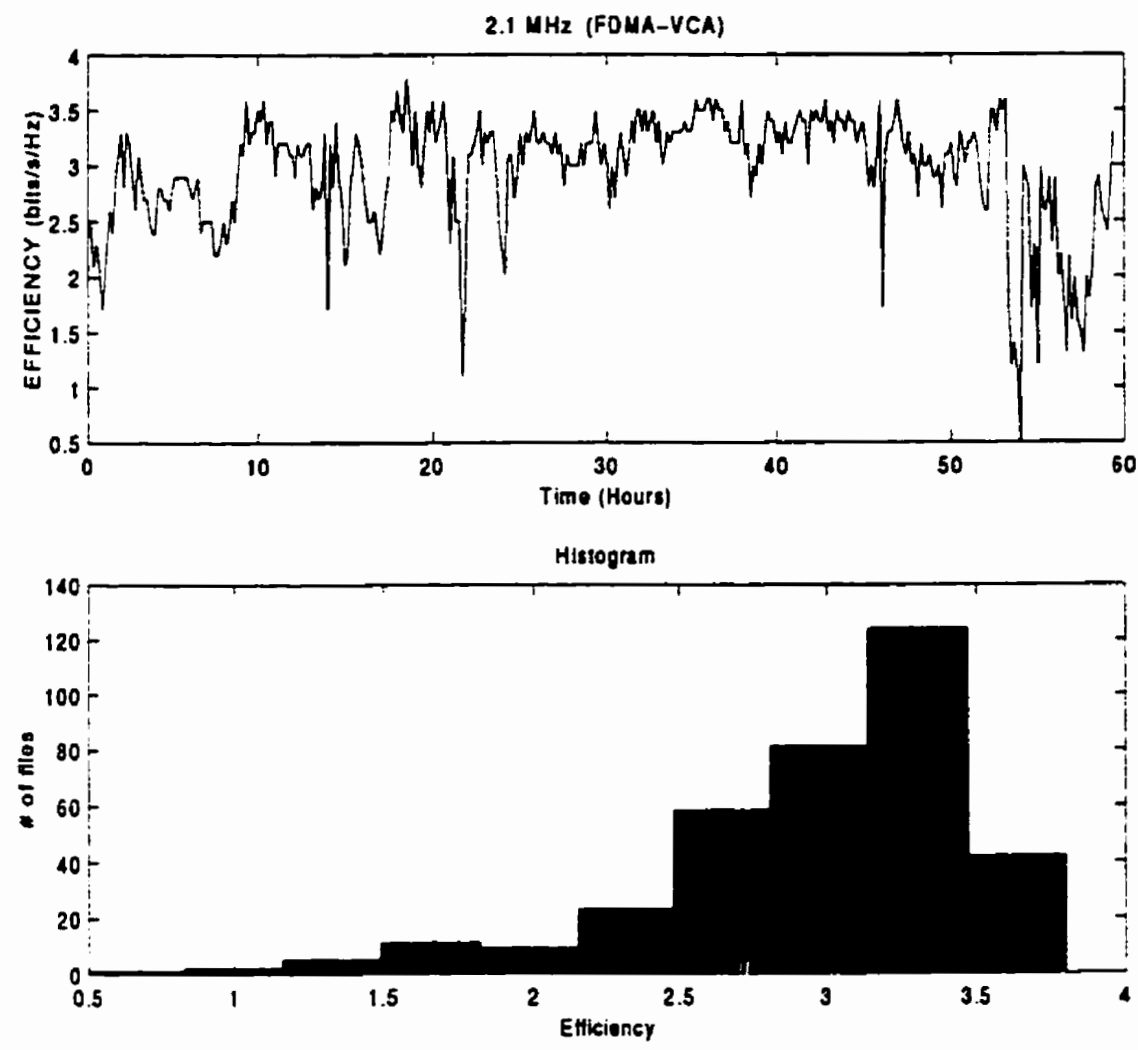

Figure 3.8: Efficiency obtained when conducting an analysis with a $2.1 \mathrm{MHz}$ FDMA-VCA scheme for site 2. The top graph shows the efficiency obtained on a 10 minute basis. The bottom graph gives an histogram using the same parameters. 
Figure 3.8 was included to show the results on a sample ingress file basis. It shows the average efficiency that would be obtained over the $5-35 \mathrm{MHz}$ spectum of site 2 using a $2.1 \mathrm{MHz}$ FDMA system with a VCA scheme. In this case, the first 60 files (1 hour) of site 1 are shown and each sample point represents the average efficiency that was obtained at that time. This figure also demonstrates the time variability of the noise by noting the variation between each consecutive sample point.

\subsubsection{Distorted channel model}

As described in Chapter 2, the channel is nearly ideal except for a few reflections. We have used a two taps channel response to model the upstream channel (equation 3.4), where $t_{o}$ and $a$ correspond to the delay and magnitude parameters found for the reflections.

$$
H(f)=1+a e^{-j 2 \pi f t_{0}}
$$

We now repeat the same procedure as for the linear model except that the channel distortion is taken into account. The parameter $H(f)$ is added to the previous equation to evaluate the SNR in each sub-band and will vary according to the two taps channel response.

$$
S N R_{f_{b b}}=\left[\frac{\sum_{f_{o b}}|H(f)|^{2} \Delta f}{\sum_{f_{o b}} N(f) \Delta f}\right]
$$

Note that there are no fixed transmitting and receiving flltcrs fur this model 
since MCM digital implementation using the FFT would not require these filters for the individual sub-bands.

\subsection{Wideband infinite DFE (WDFE) model}

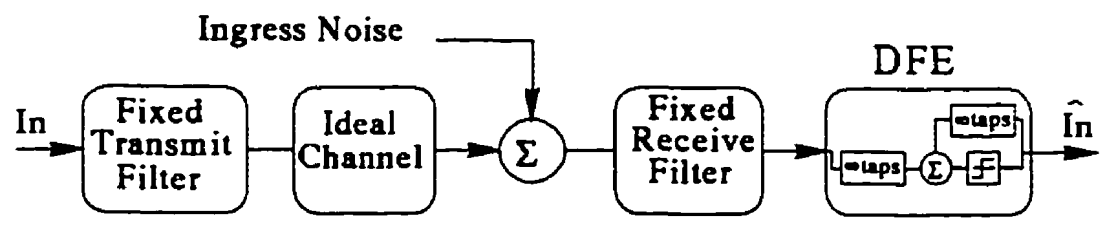

Figure 3.9: Infinite taps DFE model used for analysis.

Figure 3.9 represents the model that was used in our analysis. The purpose of this model is to bound the performance of the infinite DFE case. We will then use these results for comparison with the finite case; that is, a DFE with matched filter input and infinite numbers of forward and feedback taps. Details and characteristics of the DFE are given in the finite DFE section. For the infinite case, we adapted an equation derived by Salz [15], which expresses the equalizer's output signal to noise and ISI ratio in terms of noise and channel spectral characteristics (equation 3.6) using the minimum mean square error ( MMSE) criterion.

$$
S N R_{\infty}=-1+\exp \left\{T \sum_{n}\left[\ln \left(1+\frac{|H(f+n \Delta f)|^{2}}{N(f+n \Delta f)}\right)\right] \cdot \Delta f\right\}
$$


In the ideal case, $H(f+n \Delta f)=1$. For the channel distorted by reflections, we have used the same two taps model as described in section 3.2.4.2. Note also that we have used ideal fixed transmitting and receiving filter (i.e., no excess bandwidth).

\subsubsection{Evaluation of spectral efficiency}

As with the MCM scheme, ideal rectangular spectra channels were used to simplify the calculations. With the calculated SNR in each of the (wide) sub-bands, the best modulation scheme was selected to evaluate the spectral efficiency of each sub-band. Again, by multiplying this efficiency by the width of each sub-band and adding each total, we can get the capacity in bits per second. The same parameters as the MCM spectral efficiency analysis were used to evaluate the capacity for both the FCA and VCA scheme, as well as the ideal and distorted channel. Note that the "Wideband DFE" system and the MCM system have the same overall bandwidth (30 MHz).

\subsection{Wideband Finite DFE (WFDFE) model}

Figure 3.10 shows the model used to perform our evaluation. In contrast with the other previous models, we have considered the effect of fixed receiving and transmitting filters, of adjacent channel interference and of guardbands (carrier spacing). The purpose of this model is to find the number of taps that would be required for an implementation representative of one encountered in 


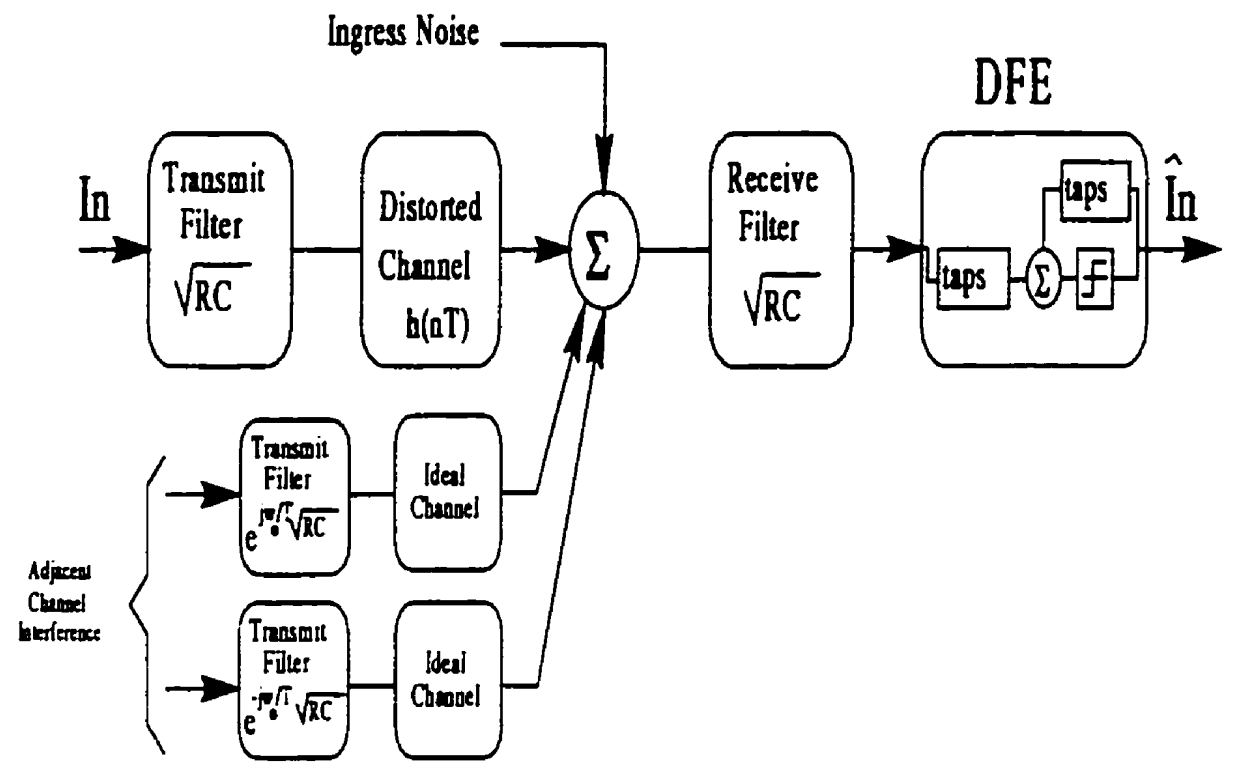

Figure 3.10: Wideband system with finite DFE model.

practice. It is also to view the effect of various combinations of parameters such as carrier spacing, fractionally spaced equalization, excess bandwidth, and guard bands on spectral efficiency.

\subsubsection{DFE characteristics}

The DFE is a good compromise when an optimum scheme such as the Viterbi equalizer is too complex to recover the data in a distorted channel. The DFE can be used to mitigate channel distortion as well as adjacent channel, cochannel and intersymbol interference [19]. The motivation for using a decision feedback equalizer over a linear equalizer is that we can improve reception of the 
current data symbol by subtracting the ISI caused by the past decision from the current symbol. The fact that the DFE bases itself on previous decisions renders the DFE prone to error propagation. But, as was mentioned in [21] and [19], this issue is not a serious problem for channels that are not severely impaired.

\subsubsection{Finite DFE evaluation details}

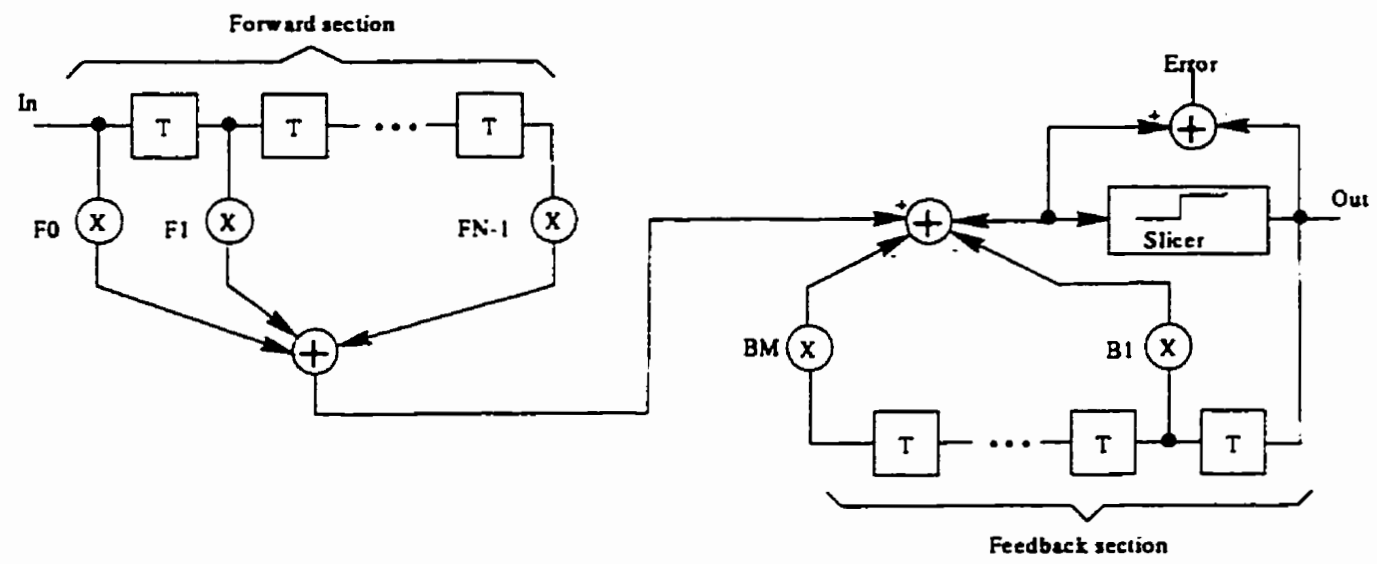

Figure 3.11: Finite DFE equalizer.

The DFE equalizer consists basically of a forward filter and a feedback filter (figure 3.11). The forward section is identical to a linear equalizer. The feedback section is responsible for removing the ISI caused by the previous symbols at the decision instant. For this model, the mean square error criterion was used to compute the taps coefficients. Equation [3.7] gives the expression used 
to compute the MMSE [19].

$$
M S E=1-X^{*} \Phi^{-1} X
$$

where

$$
\begin{gathered}
X \equiv\left[\begin{array}{c}
x(-N T) \\
\cdot \\
\cdot \\
x(0) \\
\cdot \\
\cdot \\
x(N T)
\end{array}\right] \\
\Phi \equiv A-D D^{*}
\end{gathered}
$$

and $A$ and $D$ are defined by:

$$
\begin{gathered}
A \equiv\left[\left\{A_{l m}\right\}_{-N \leq l, m \leq N}\right] \\
D \equiv\left[\left\{D_{l m}\right\}_{-N \leq l \leq N, 1 \leq m \leq M}\right]
\end{gathered}
$$

where

$$
D_{l m} \equiv x((m-l) T)
$$

and 


$$
A_{l m} \equiv \sum_{k} x((k-m) T) x^{*}((k-l) T)+R_{l m}
$$

Vector $X$ is the low pass equivalent impulse response of the channel and $R_{l_{m}}$ is the additive noise covariance. $M$ and $N$ correspond to the number of forward and feedback taps respectively.

\subsubsection{The additive noise covariance}

The additive noise covariance was found using the Wiener - Khinchin theorem [20] by taking the inverse Fourier transform of the ingress spectra files (equation 3.9). $N$ is the number of sample points in the sub-band and $S(f+n \Delta f)$ is the sample point taken from the ingress spectra file in the corresponding sub-band.

$$
R(k)=\frac{1}{N} \sum_{n} S(f+n \Delta f) e^{j 2 \pi n \Delta f k T}
$$

Note here that because of the $100 \mathrm{kHz}$ resolution for the spectrum analyzer, there is a limit imposed on the number of taps for the forward section of the finite DFE. For example, for a $1 \mathrm{MHz}$ wide sub-band with symbols spaced at $T=1 * 10^{-6}$ seconds, the tap limit is 5 . Otherwise, we will be evaluating $R(k)$ in the repeated spectrum components of $S(f+n \Delta f)$ (sampling property). 


\subsubsection{Forward autocorrelation matrix and adjacent channel in- terference}

To implement the adjacent channel interference, the following equations were used for the 3 impulse responses in the model: the impulse response of the channel $h_{0}$, and the 2 impulse responses for the adjacent channel interference $h_{1}$ and $h_{2}$. The square root of raised cosine impulse response $\gamma$ is shifted in frequency and then convolved for the adjacent interference impulse response.

$$
\begin{gathered}
h_{0}=x(t) \star \gamma(t) \\
h_{1}=\gamma(t) \star\left(\gamma(t) \cdot e^{j 2 \pi f_{g} t}\right) \\
h_{2}=\gamma(t) \star\left(\gamma(t) \cdot e^{-j 2 \pi f_{g} t}\right)
\end{gathered}
$$

For the MMSE calculations, we have evaluated the autocorrelation of each impulse response (A matrix) to form the forward autocorrelation matrix $\mathrm{A}=\mathrm{A} 0+\mathrm{A} 1+\mathrm{A} 2$ (refer to equation 3.8 ). The same procedure is then applied to compute the MMSE.

\subsubsection{Capacity with excess bandwidth and guardbands}

Guard bands are implemented by varying the frequency shift $\left(f_{g}\right)$ in equation 3.10 and 3.11. The analysis was conducted considering various excess bandwidth and frequency shift parameters. Figure 3.10 illustrates the case for $50 \%$ 
excess bandwidth filters with an overlapping factor of $50 \%$, meaning that the carriers are spaced exactly $1 / \mathrm{T}$ apart. A simple relation can be use to evaluate the overlapping factor (OF) in percentage.

$$
O F=\left(1-f_{g}\right) * 100-\alpha
$$

$\alpha$ is the excess bandwidth in percentage and $f_{g}$ is the frequency shift used to implement the guardbands.

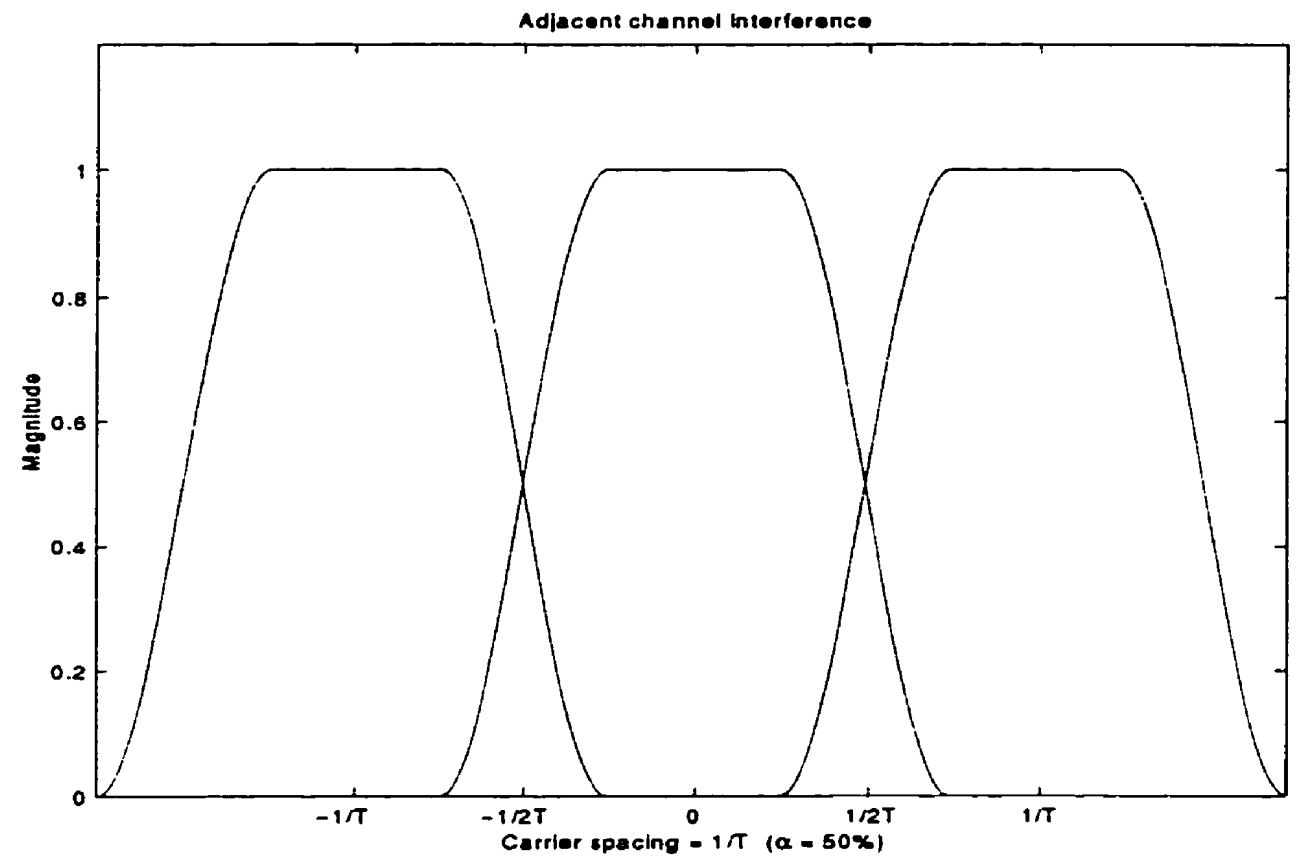

Figure 3.12: Equivalent spectra for 3 sub-bands with $50 \%$ excess bandwidth and $1 / \mathrm{T}$ carrier spacing.

To evaluate the capacity, the efficiency was computed for each individual sub-band. If guardbands were used, that portion of the spectrum was consid- 
ered when the total capacity of the spectrum was computed. For example, for $10 \mathrm{MHz}$ sub-bands, we have 3 sub-bands and 2 guardbands for the $5-35 \mathrm{MHz}$ spectrum. If the carriers are spaced at $1 / \mathrm{T}+25 \%$, then we add $2^{*} 10^{*} .25$ to $30 \mathrm{MHz}=35 \mathrm{MHz}$. The average efficiency in bps/Hz is found by computing the efficiency including each sub-band, thus dividing by $35 \mathrm{MHz}$ instead of $30 \mathrm{MHz}$. The capacity of each sub-band is evaluated individually, considering the effect of adjacent channel interference on both sides. In this example, the last sub-band from 25-35 $\mathrm{MHz}$ would be affected by the adjacent sub-band from $35-45 \mathrm{MHz}$ and from $15-25 \mathrm{MHz}$. In the case of site 2, where the results were only taken from the $5-35 \mathrm{MHz}$ portion of the spectrum, the portion of spectrum from $25-35 \mathrm{MHz}$ is duplicated into the missing $35-45 \mathrm{MHz}$ portion. This procedure closely resembles a system encountered in practice since the spectrum is filtered through the square root of raised cosine filters and because these portions of the spectrum are almost identical.

\subsubsection{Evaluation of spectral efficiency}

Except for the evaluation of the SNR, the exact same approach as seen in the MCM scheme will be used to evaluate the spectral efficiency. The same modulation and allocation schemes will also be used.

We have used the following definition to compute the SNR, taken from [15], and [22], and [23]:

$$
S N R=\frac{1-m m s e}{m m s e}
$$


Note that for the $100 \mathrm{kHz}$ sub-band (the frequency resolution of the spectrum analyzer), the MCM scheme and the WDFE or WFDFE will give identical results. This fact can be seen by looking at the equation for the infinite DFE. If we are at the spectrum analyzer resolution, only one sample point will be used to compute the SNR. We can remove the summation in the equation 3.6 which leaves us with:

$$
S N R_{\infty}=\frac{|H(f+n \Delta f)|^{2}}{N(f+n \Delta \tilde{f})}
$$

This is identical to the equation for the MCM with $100 \mathrm{kHz}$ sub-bands. 


\section{Chapter 4}

\section{Results and analysis}

\subsection{Introduction}

In this chapter, we give the results of return band capacity for the multicarrier modulation and for a wideband system using adaptive decision feedback equalization. The first section of this chapter will show how using various sub-channel bandwidths as in FDMA and FDMA/TDMA schemes can affect the parallel modulation scheme. In addition, the time varying aspect of the channel is considered using fixed and variable bit rate allocation to sub-bands as in MCM with frequency division multiplexing. The same analysis is then repeated in the second section for a WDFE contending with ingress noise instead of channel distortion. Note that results have been computed for the same sub-band widths in both cases in order to view the effect of the subband widths on the performance of both systems. The reader should take into 
account that a subscriber transmitting upstream in an MCM system would use narrowbands in the order of $\mathrm{kHz}$; on the other hand, in a WDFE system, transmission would be time-shared in a single band of several $\mathrm{MHz}$ or tens of MHz. The MCM system can thus be viewed as a parallel modulation method and the WDFE system can be viewed as a serial modulation method. In the third section, we compare the capacity of the MCM system with the WDFE system for the FCA and VCA schemes. The last section of this chapter has been dedicated to a more detailed analysis of the WDFE since this system is of low complexity and would present results similar to the performance of the MCM system. The required number of taps, the effect of guardbands, adjacent channel interference, and the receiving and transmitting filters have been included to resemble a system encountered in practice. Note that since most of the noise energy is located at the lower end of the spectrum, most of the modulation schemes in that region do not meet the required performance objectives. Consequently, the total spectral efficiency for a $30 \mathrm{MHz}$ spectrum is affected by this degradation and presents poor performance. For this reason, we evaluated the capacity considering the $5-35 \mathrm{MHz}, 5-15 \mathrm{MHz}$, and 15-35 $\mathrm{MHz}$ portions of the spectrum. 


\subsection{MCM}

\subsubsection{Effect of the sub-band width on capacity}

Our first step was to determine the effect of the sub-band width on capacity for the MCM system. In this section we assume a system with an ideal rectangular channel and additive piecewise constant noise in each sub-band. The capacity was evaluated as defined in section 3.2.2 for the FCA and VCA scheme with the parameters of $1 \%$ outage and the BER less than $10^{-6}$ and $10^{-9}$. The subband widths of $.1, .2, .5,1,2,5$ and $10 \mathrm{MHz}$ were used to view the effect. Each sub-band receiver, irrespective of bandwidth, uses a simple detection scheme that assumes its noise is white. Thus the MCM scheme diverges more and more from optimality as the sub-band widths increase. Note that no power optimization was needed in this approach since it has already been shown that

a scheme like MCM optimally distributes the power more or less constantly in the sub-bands with high SNR [12]. Thus the performance of MCM would not be greatly enhanced by power optimization. In the next section, we further discuss FCA versus VCA performance.

The graphs

Except for example 4.2.1.1, all the figures in this section can be interpreted as follows: the vertical left axis represents the efficiency in bits per second per hertz (bps/Hz); the vertical right axis represents the total capacity for the portion of the spectrum under study; and the bottom horizontal axis represents each sub-band width that was used to compute the capacity. Each 
graph contains two curves corresponding to the BER of $10^{-6}$ and $10^{-9}$ plotting the efficiency versus the sub-band width. The figures in example 4.2.1.1 can be interpreted as follows: the vertical axis represents the bit allocation in bps $/ \mathrm{Hz}$ and the horizontal axis represents the frequency from $5-35 \mathrm{MHz}$.

\subsubsection{An example of bit rate allocation}

The following results were included to illustrate the bit rate allocation in each sub-band versus frequency for a FCA scheme. Because this example is for illustrative purposes, only one case using $2 \mathrm{MHz}$ sub-bands with an outage parameter of $1 \%$ and a BER of $10^{-6}$ is shown. Figure 4.1 and figure 4.2 show the allocation for $2 \mathrm{MHz}$ sub-bands over the $5-35 \mathrm{MHz}$ portion of the spectrum for site 1 and site 2 respectively. Each bullet represents the bit rate that was allocated in each $2 \mathrm{MHz}$ wide sub-band. Notice that with the imposed BER and outage requirements, the first $5-15 \mathrm{MHz}$ portion is unusable in both sites when we used $2 \mathrm{MHz}$ sub-bands. In the next sub-section, we will consider the effect of sub-band widths on capacity for the FCA and VCA scheme.

\subsubsection{5-35 $\mathrm{MHz}$ portion}

Figure 4.3 and figure 4.4 show the results for site 1 and site 2 respectively for the FCA scheme. Figure 4.5 and figure 4.6 show the results for the VCA scheme. We can see that there is a tendency of better performance rates with smaller sub-band widths for both sites, and for both BERs, when using the VCA scheme. In figure 2.4 and figure 2.5 , notice that site 2 has better noise 


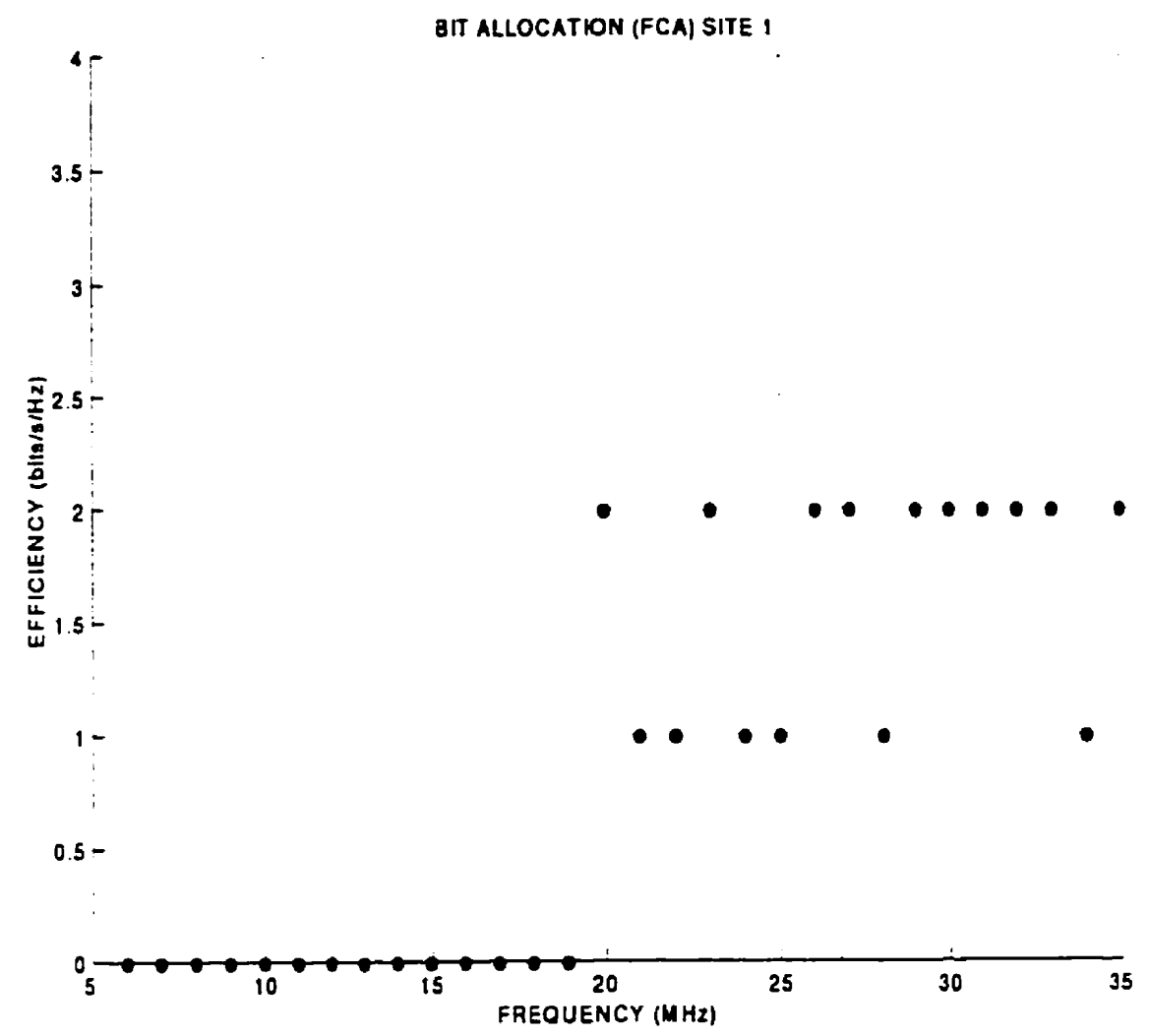

Figure 4.1: Bit rate allocation for the $5-35 \mathrm{MHz}$ spectrum of site 1 versus frequency for a FCA scheme. 


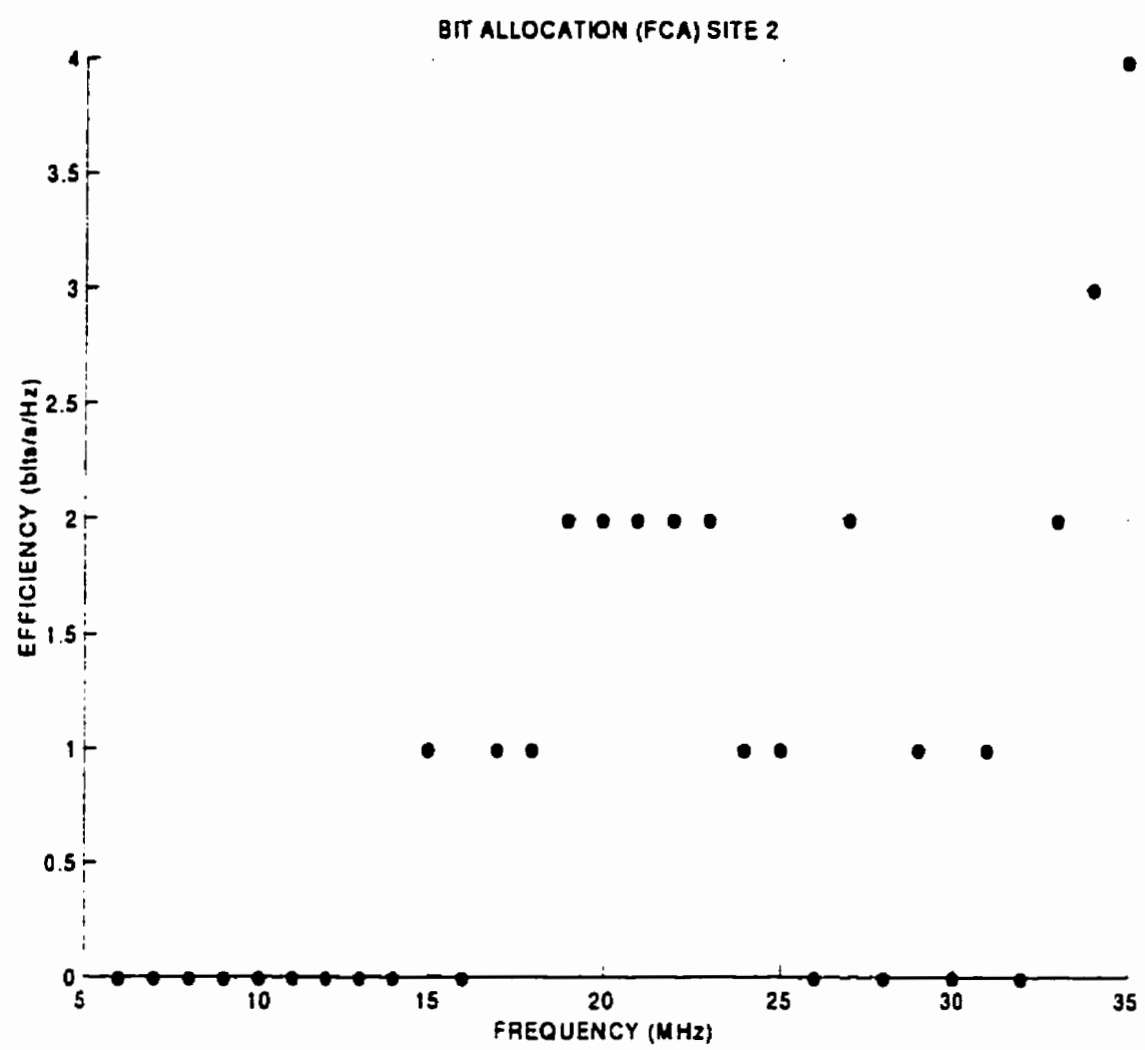

Figure 4.2: Bit rate allocation for the $5-35 \mathrm{MHz}$ spectrum of site 2 versus frequency for a FCA scheme. 
characteristics for the first 5-15 $\mathrm{MHz}$ portion of the spectrum. In the case of fixed channel allocation for site 1, the usage of the FCA scheme does not show better results when using smaller sub-bands. This phenomena is caused by the $1 \%$ outage requirements and the inherent characteristic of FCA. If you consider the 2000 sample files and an outage of only 1\%, at least 1990 files must meet the SNR requirements to use a particular modulation scheme in a particular location. But if you consider wider sub-bands, there is a possibility that more files will meet the SNR requirements since the noise will average out over the sub-band. It also explains why we have better performance using smaller sub-bands in the case of the VCA scheme, since in this case we can effectively match the slowly varying ingress noise of the spectrum.

\subsubsection{5-15 $\mathrm{MHz}$ portion}

Figure 4.7 and figure 4.8 show, for the lower end portion of the spectrum, the results for the FCA scheme for site 1 and site 2 respectively. Figure 4.9 and figure 4.10 show the results for the VCA. Note that the noise was so high in that portion of the spectrum that none of the schemes were reliable enough to meet the required BER of $10^{-6}$ and $10^{-9}$ in the case of the FCA scheme for site 1. This means that in this region of the spectrum, extensive coding would need to be used for transmission. We can see that we have a tendency of having better performance with smaller sub-band widths for both sites and both BERs. This shows the importance of effectively matching the noise and the bit rate for this portion of the spectrum. 


\subsubsection{15-35 $\mathrm{MHz}$ portion}

Figure 4.11 and figure 4.12 show the results for the FCA for both sites. Figure 4.13 and figure 4.14 show the results for the VCA scheme. Again we can see that there is a tendency of having better performance with smaller sub-band widths for both sites and for both BERs for the VCA scheme. Note here the large improvement in efficiency compared to the lower portion of the spectrum.

Here we can also see the same irregularities as in the $5-35 \mathrm{MHz}$ portion (figure 4.11 and figure 4.12), where there is sometimes better efficiency through larger sub-band width. These results are caused by the inherent characteristic of the FCA scheme as mentioned in section 4.2.1.2. 


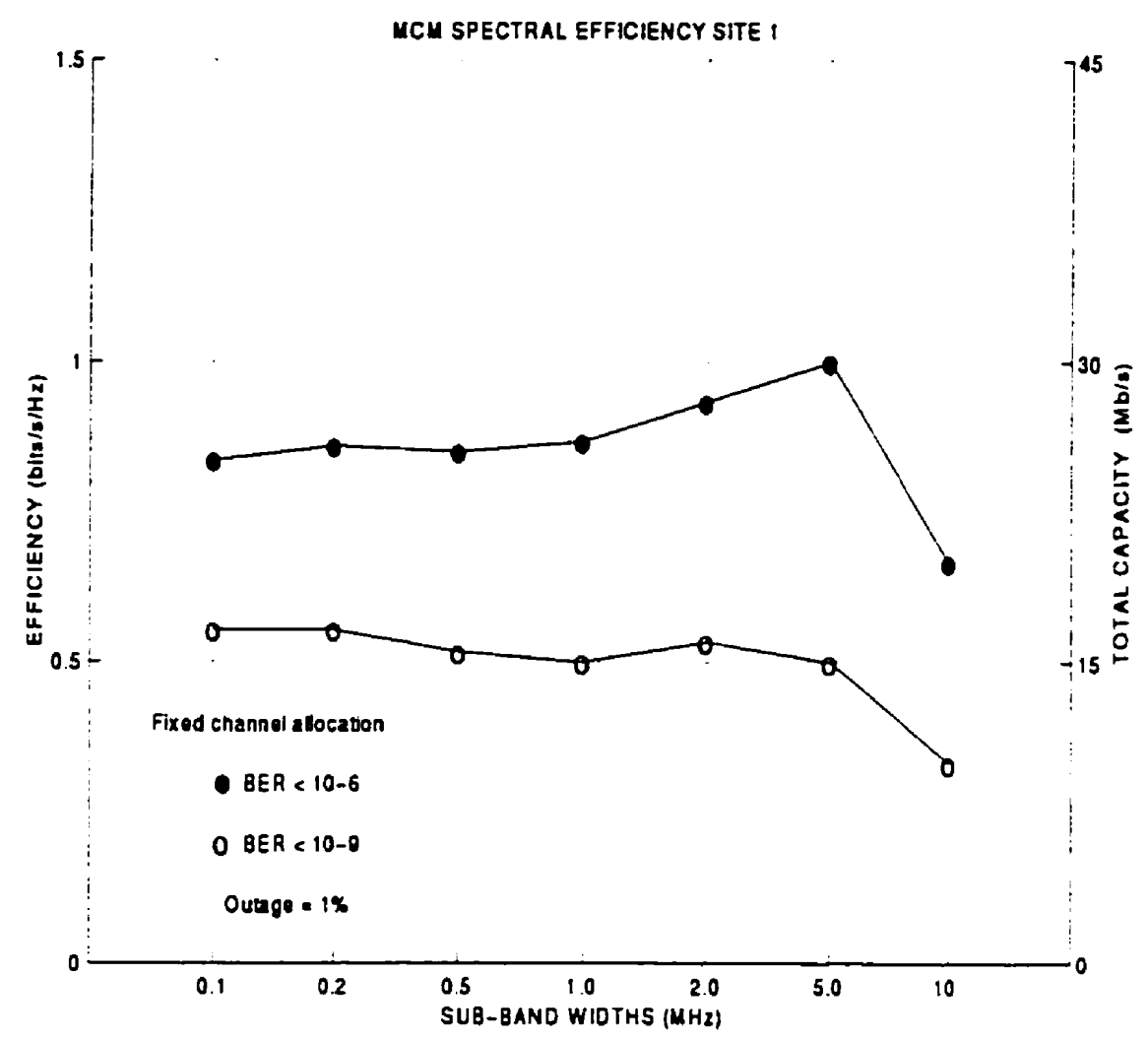

Figure 4.3: Efficiency for the 5-35 $\mathrm{MHz}$ spectrum of site 1 versus sub-channel bandwidth for a FCA scheme. 


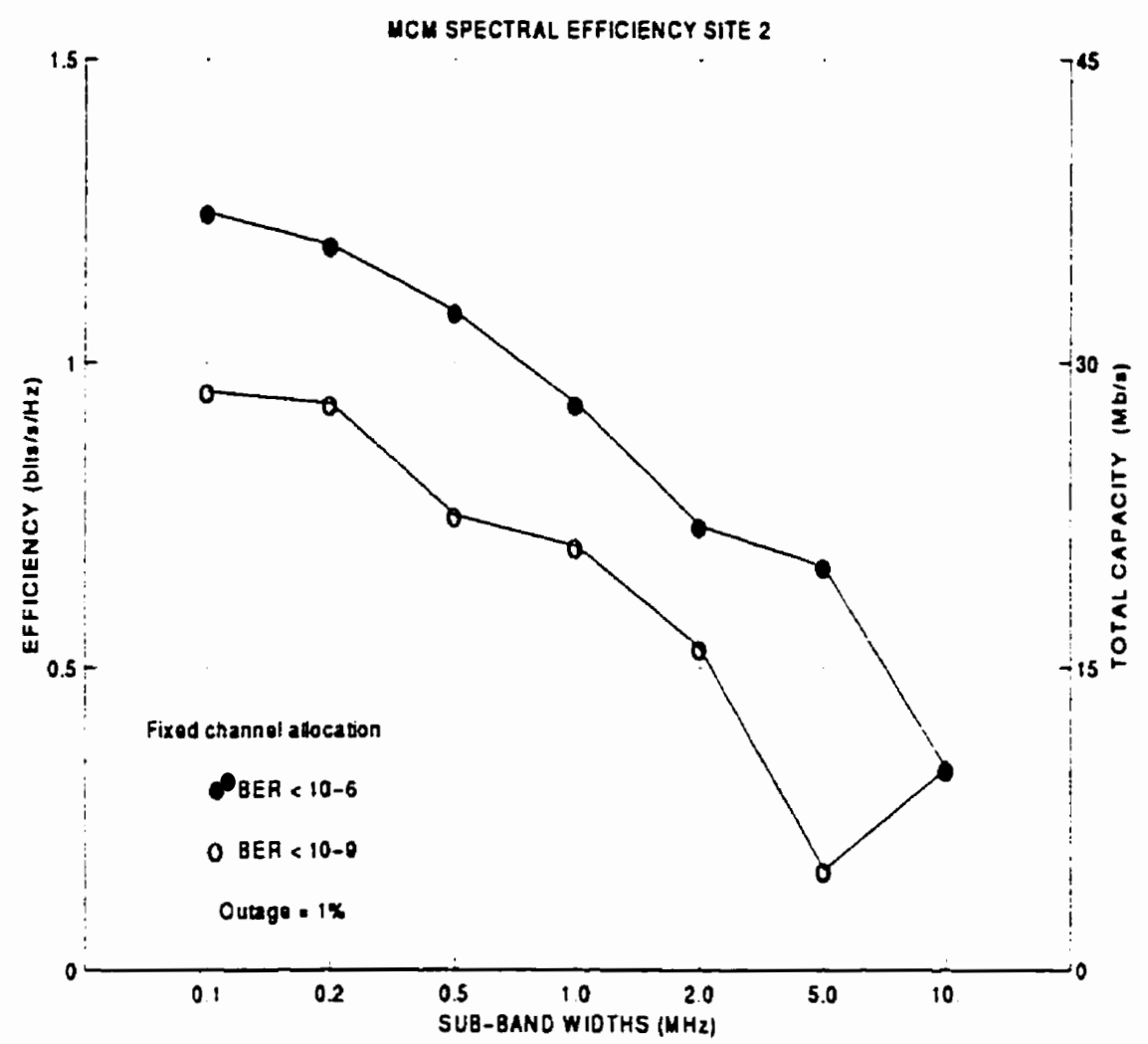

Figure 4.4: Efficiency for the 5-35 $\mathrm{MHz}$ spectrum of site 2 versus sub-channel bandwidth for a FCA scheme. 


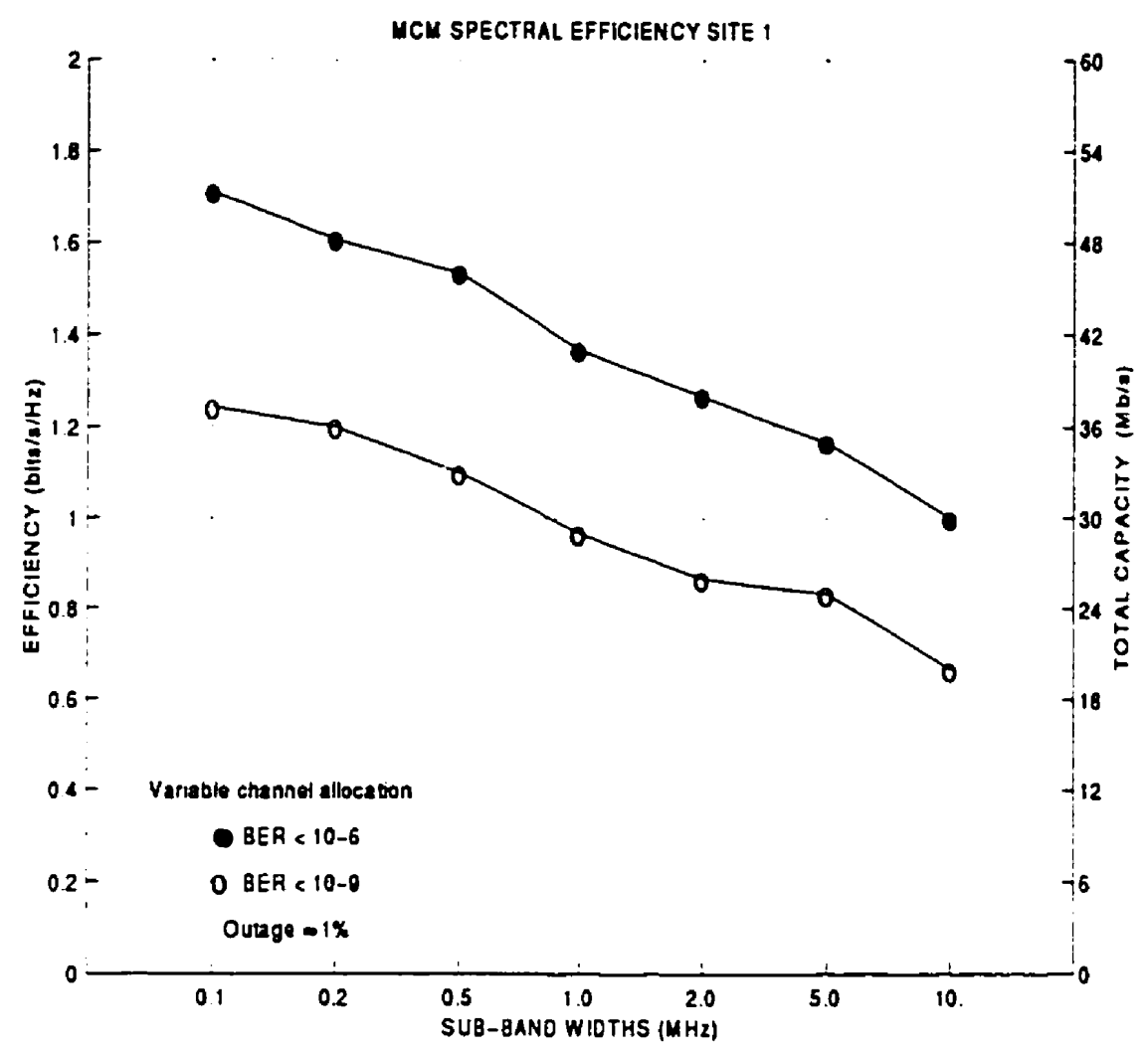

Figure 4.5: Efficiency for the $5-35 \mathrm{MHz}$ spectrum of site 1 versus sub-channel bandwidth for a VCA scheme. 


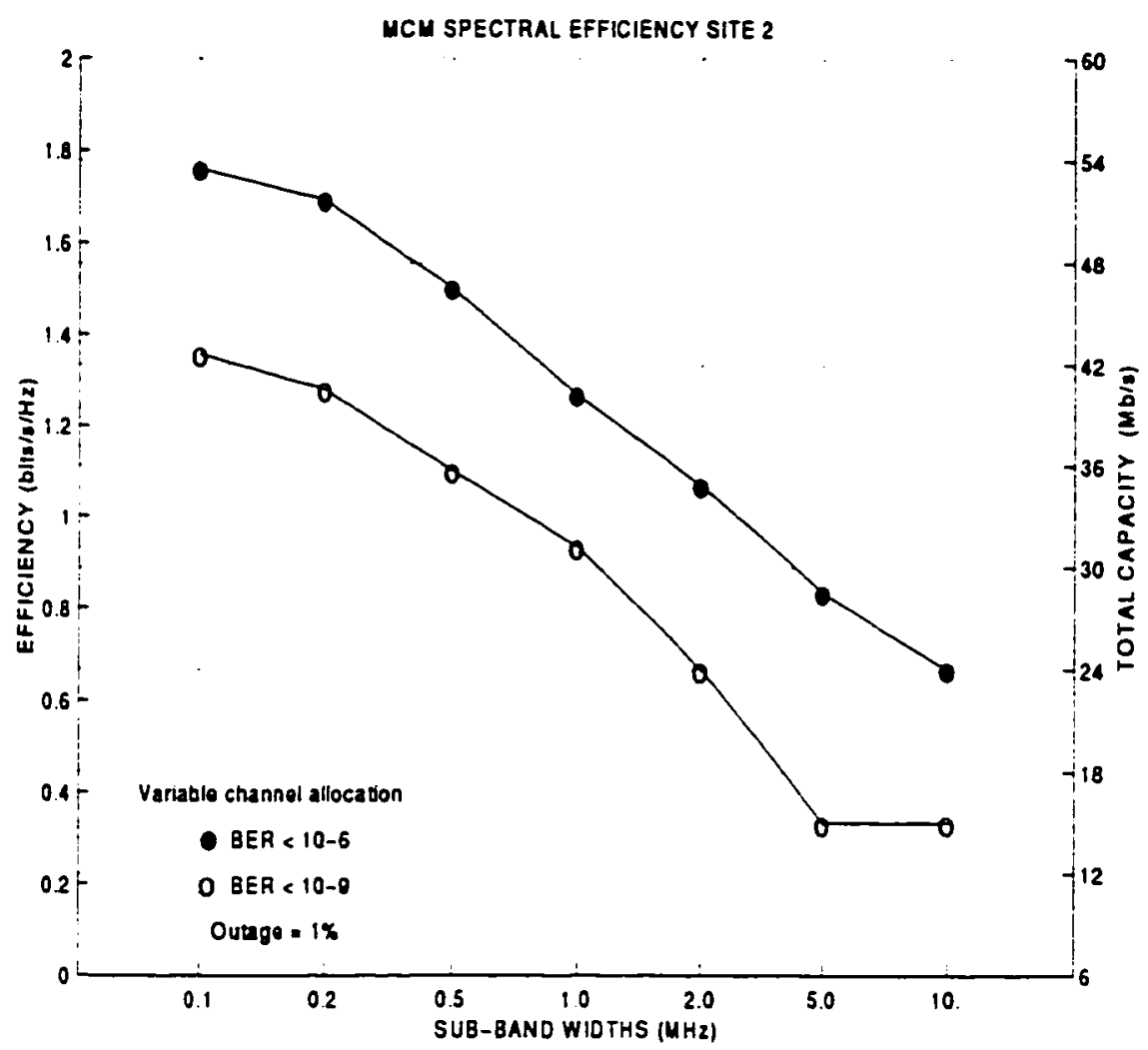

Figure 4.6: Efficiency for the $5-35 \mathrm{MHz}$ spectrum of site 2 versus sub-channel bandwidth for a VCA scheme. 


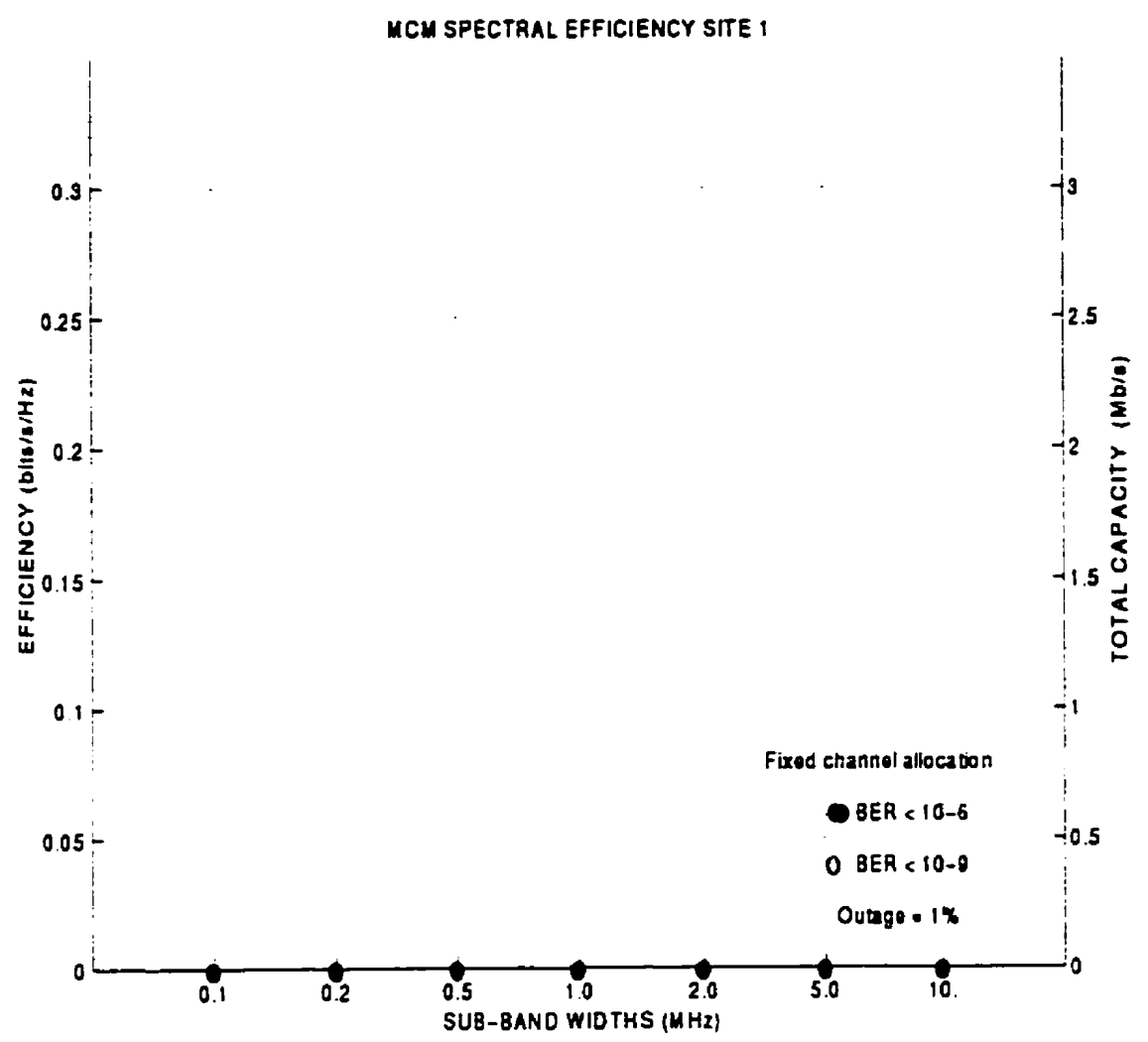

Figure 4.7: Efficiency for the 5-15 $\mathrm{MHz}$ spectrum of site 1 versus sub-channel bandwidth for a FCA scheme. 


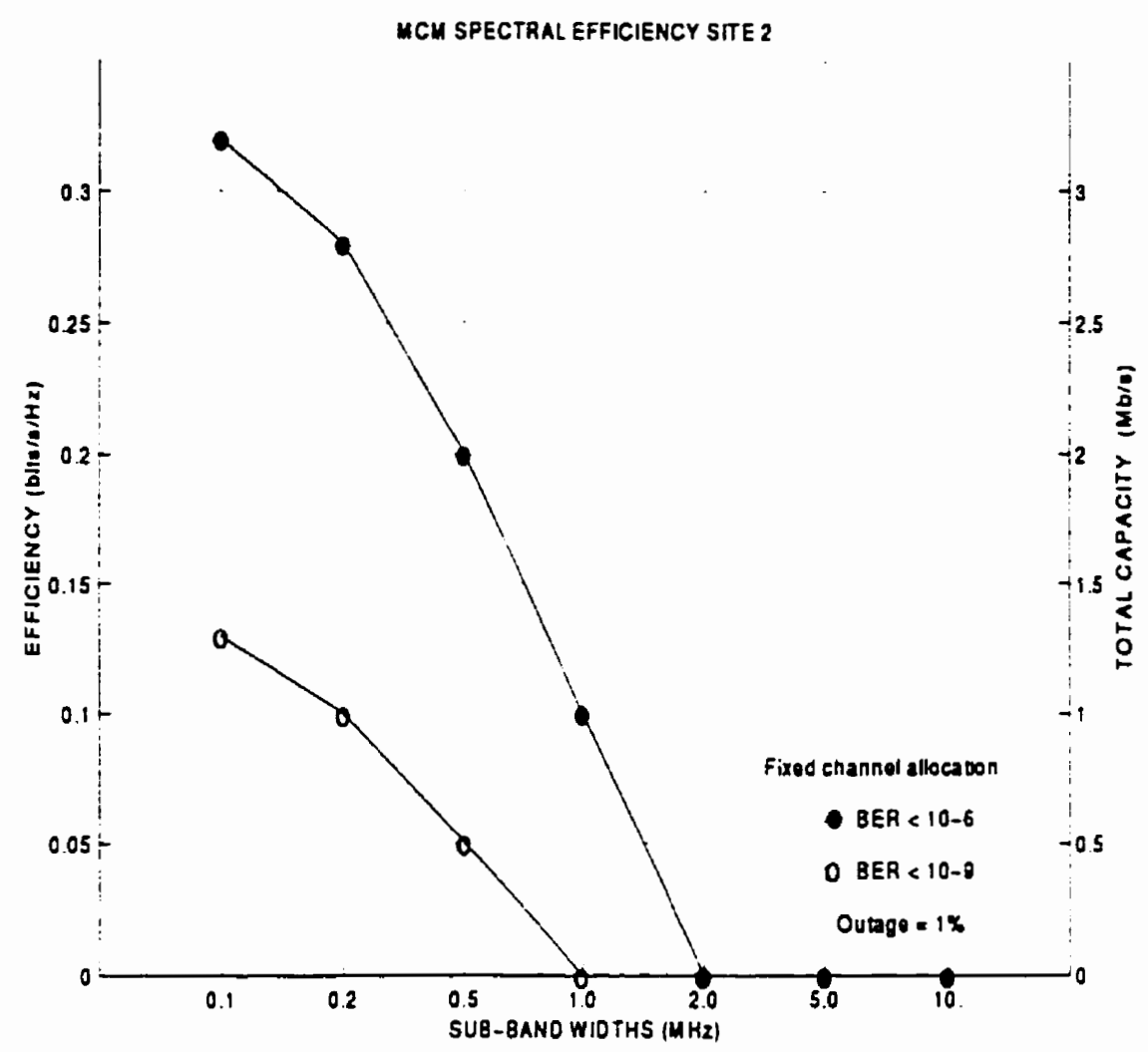

Figure 4.8: Efficiency for the $5-15 \mathrm{MHz}$ spectrum of site 2 versus sub-channel bandwidth for a FCA scheme. 


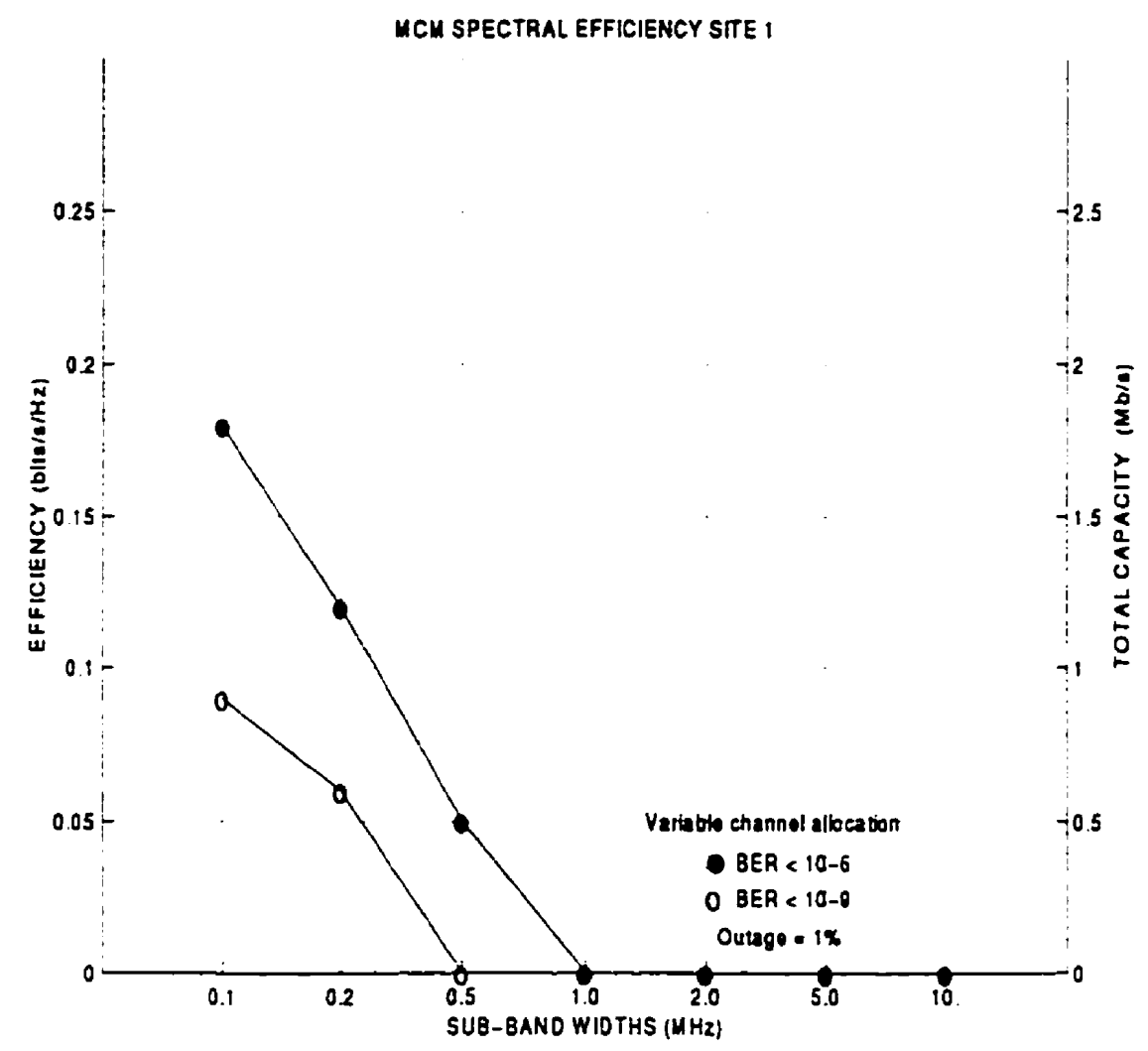

Figure 4.9: Efficiency for the $5-15 \mathrm{MHz}$ spectrum of site 1 versus sub-channel bandwidth for a VCA scheme. 


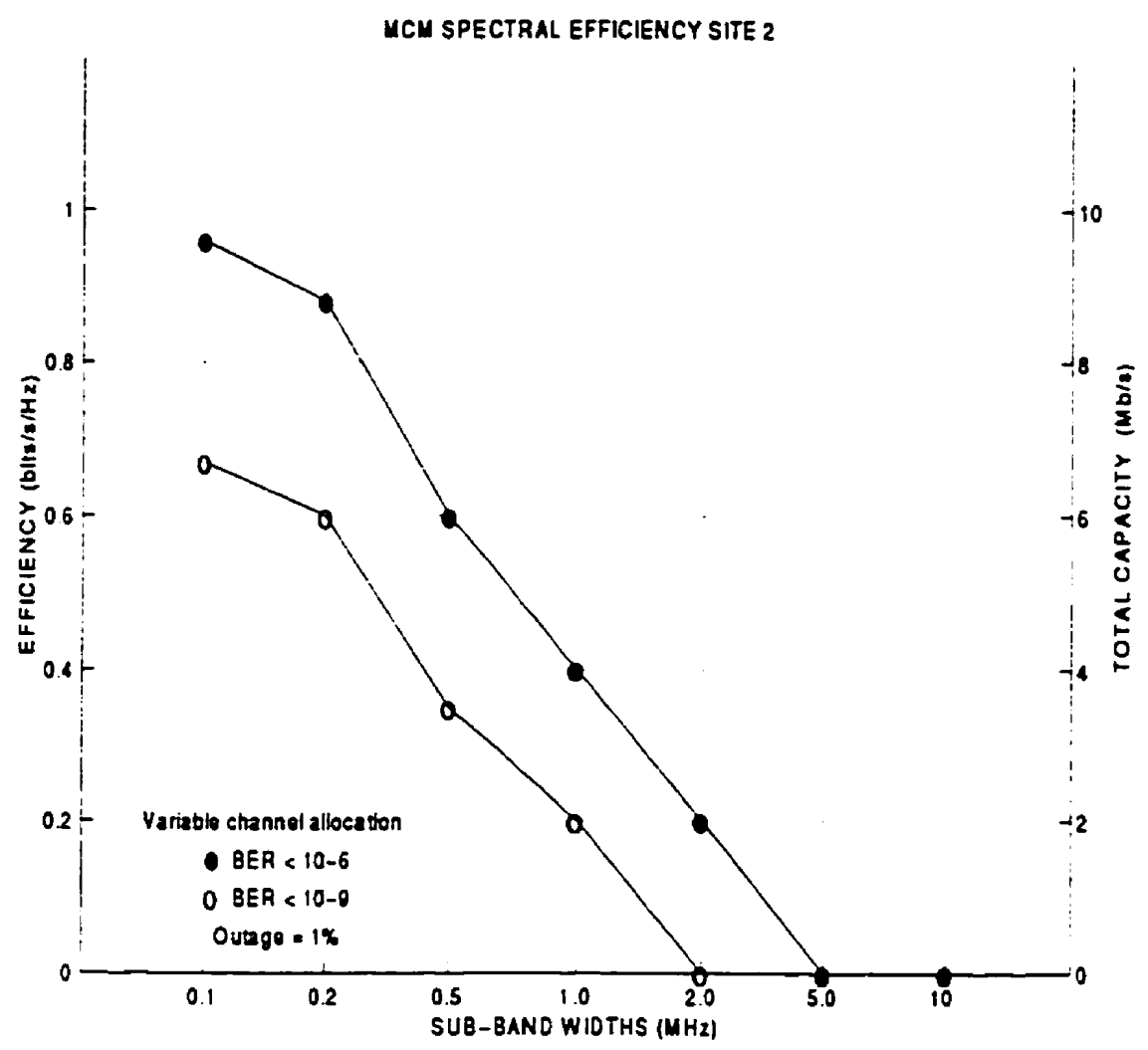

Figure 4.10: Efficiency for the $5-15 \mathrm{MHz}$ spectrum of site 2 versus sub-channel bandwidth for a VCA scheme. 


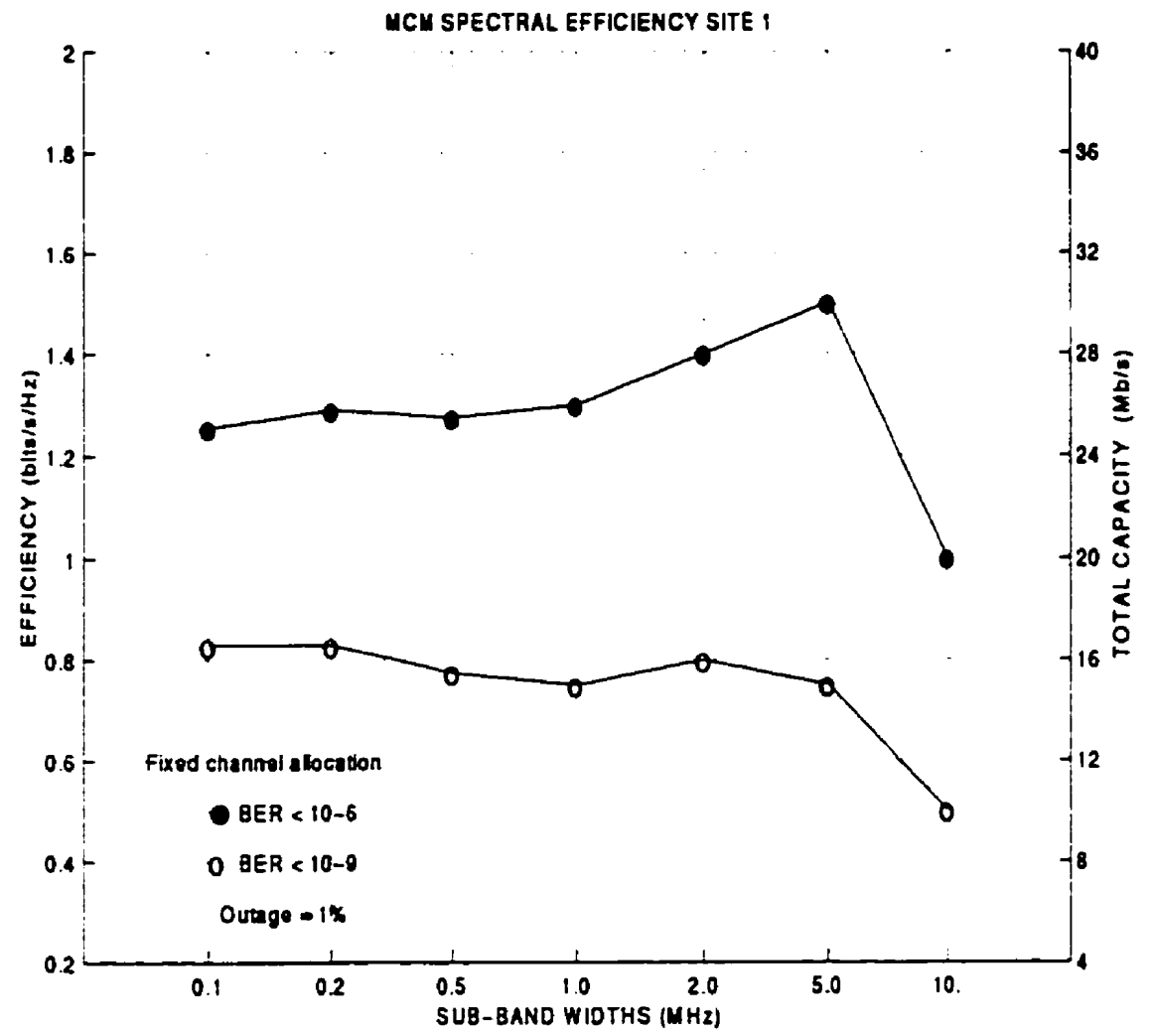

Figure 4.11: Efficiency for the 15-35 $\mathrm{MHz}$ spectrum of site 1 versus sub-channel bandwidth for a FCA scheme. 


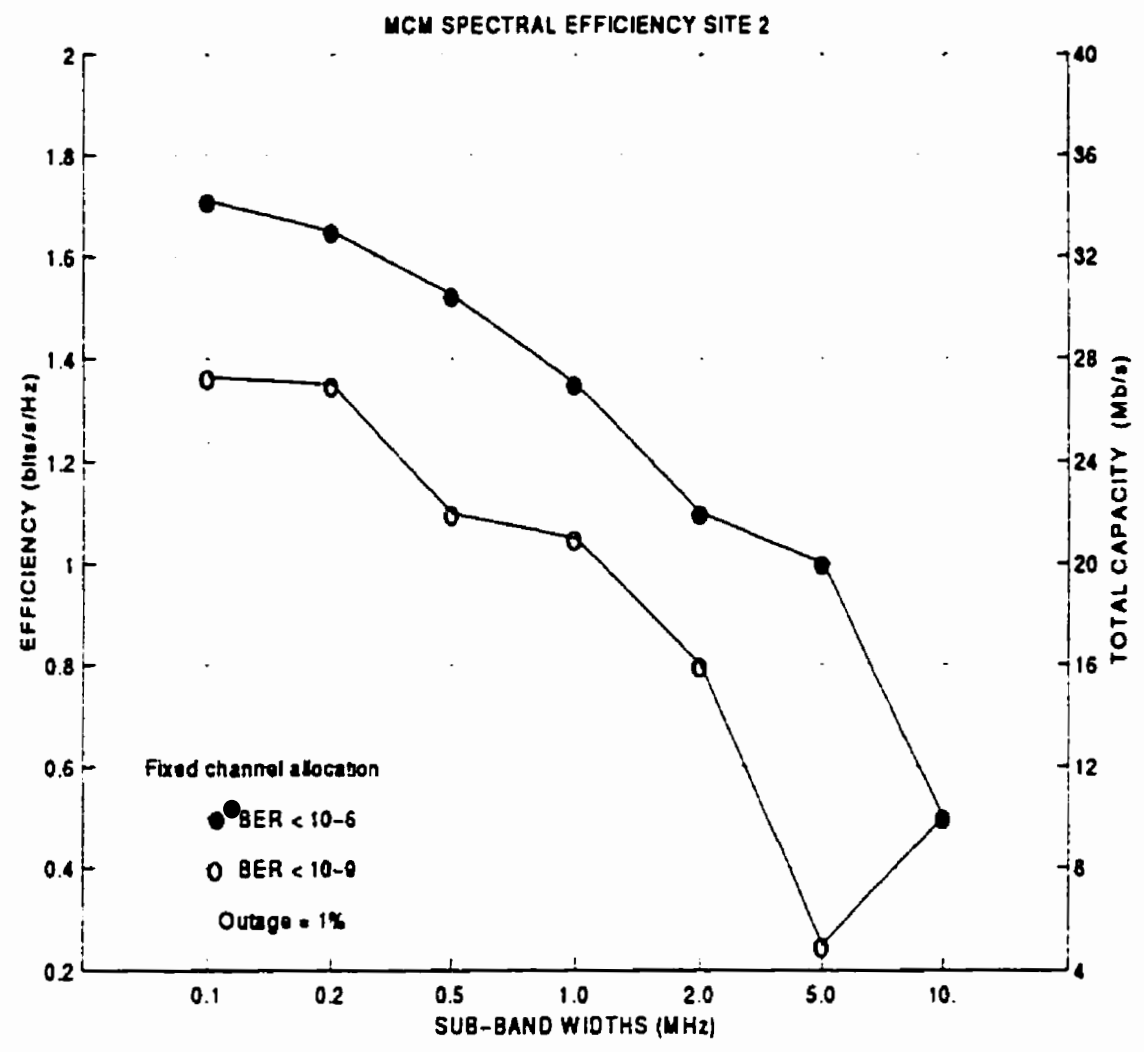

Figure 4.12: Efficiency for the 15-35 $\mathrm{MHz}$ spectrum of site 2 versus sub-channel bandwidth for a FCA scheme. 


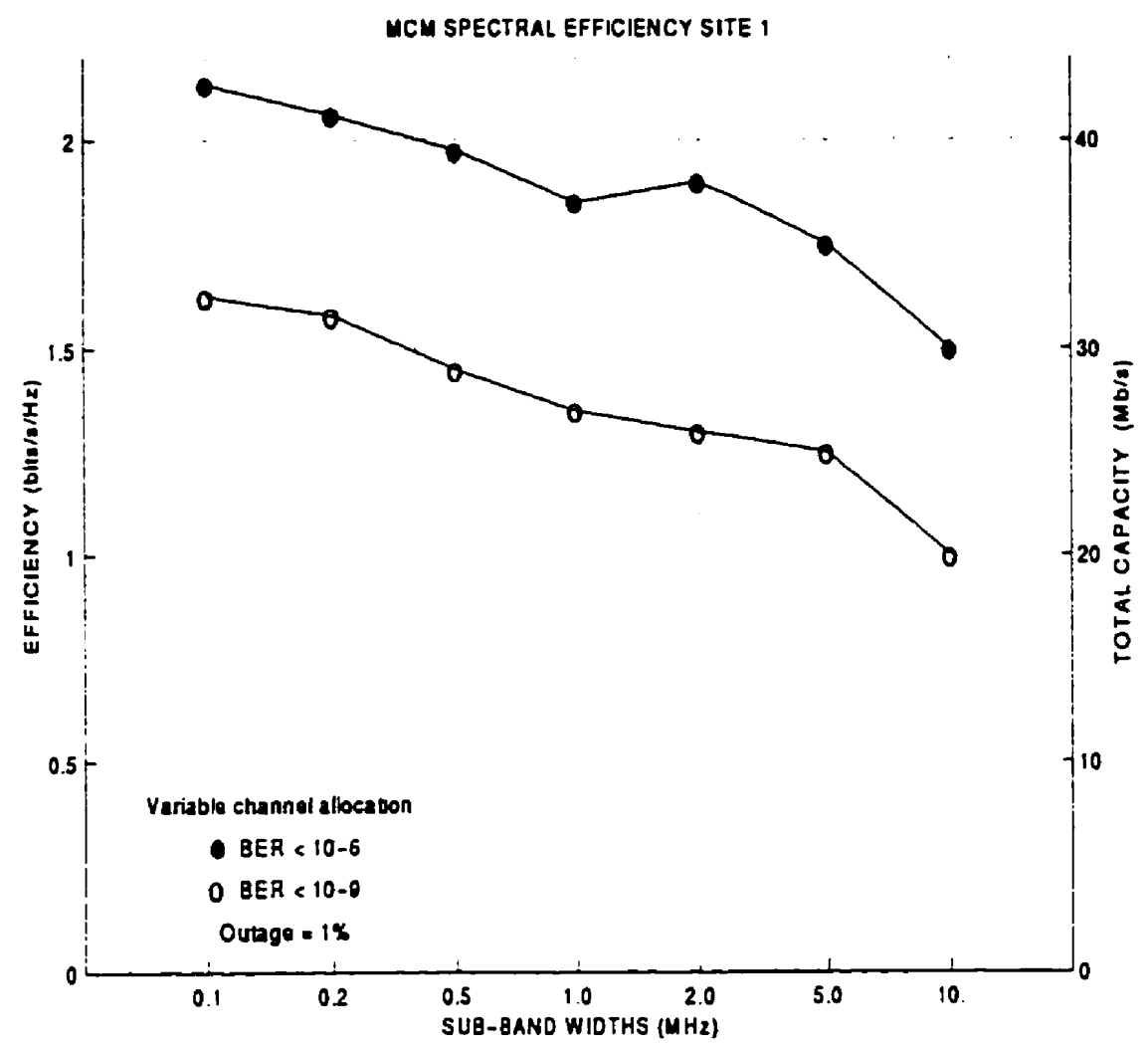

Figure 4.13: Efficiency for the 15-35 MHz spectrum of site 1 versus sub-channel bandwidth for a VCA scheme. 


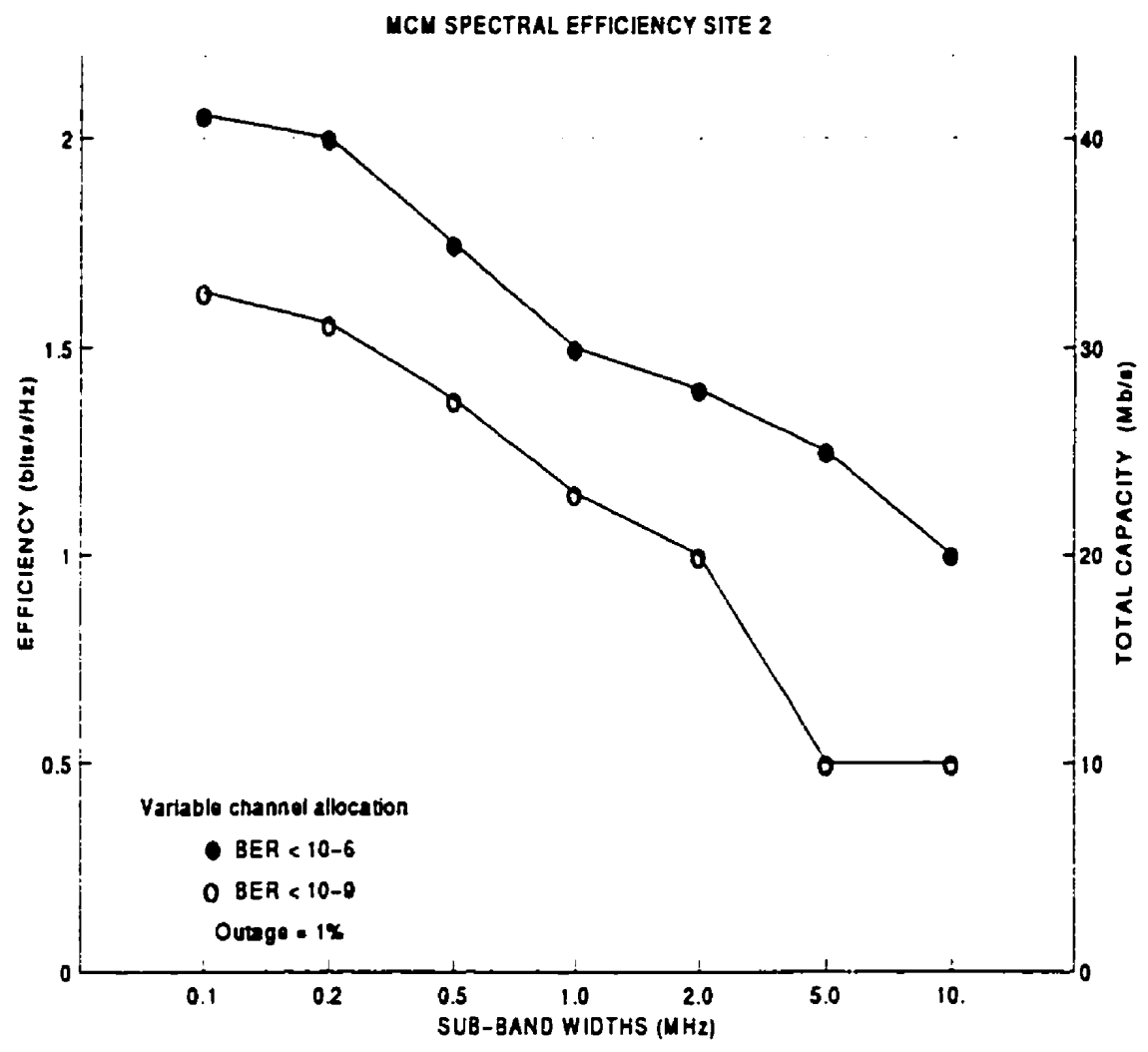

Figure 4.14: Efficiency for the $15-35 \mathrm{MHz}$ spectrum of site 2 versus sub-channel bandwidth for a VCA scheme. 


\subsubsection{FCA versus VCA for MCM}

In this section, we compare the performance of the FCA scheme with that of the VCA scheme. This will enable us to see if a significant improvement can be obtained by using a more complex scheme such as VCA. Note that for this comparison, both systems must not allow more than $1 \%$ outage. We make the assumption that the VCA method would be able to effectively adapt and remap its assignment of signal constellations to sub-bands on, for example, a minute to minute basis, and that we are dealing with slowly time varying ingress noise. We also note that the use of the VCA method implies that the aggregate achievable bit rate will vary with time. We can see by comparing the figures $4.3,4.4,4.7,4.8,4.11$ and 4.12 with figures $4.5,4.6,4.9,4.10,4.13$ and 4.14 that all portions of the spectrum demonstrate better results for VCA. Table 4.1 presents the improvement in efficiency for both sites for the sub-band width of $100 \mathrm{kHz}$. Note that the time varying allocation is also more effective in the lower end of the spectrum with the presence of more noise energy. This clearly illustrates the significant effect of time variability of noise, especially in the lower portion of the spectrum.

\subsubsection{Channel distortion by reflections}

Figure 4.15 presents the results for a channel distorted by reflections. In this case, we have assumed a $20 \mathrm{~dB}$ echo delayed by $500 \mathrm{nsec}$. We can assess that the distortion has almost no effect on the results for the MCM scheme. As seen 
Table 4.1: Performance of VCA over FCA with MCM scheme with $100 \mathrm{kHz}$ sub-bands.

\begin{tabular}{|c|c|c|c|}
\hline Site & $5-35 \mathrm{MHz}$ & $5-15 \mathrm{MHz}$ & $15-35 \mathrm{MHz}$ \\
\hline \hline 1 & $112 \%$ & $\infty$ & $75 \%$ \\
\hline 2 & $50 \%$ & $150 \%$ & $23 \%$ \\
\hline
\end{tabular}

in Chapter 2, the reflection effect results in a sinusoidal magnitude response. The order of magnitude of the reflection is not high enough to change the result for the MCM scheme. Note that a distorted channel, with reflections having echo in the order of magnitude and delay found in the cable environment, has almost no effect on capacity. Small variations are seen when the ripple effect (figure 2.6) cannot be averaged over the sub-bands; in particular, the sub-band widths of .1 .2 .5 and $1 \mathrm{MHz}$. For larger sub-band widths, the ripple effect found will be averaged out (refer to equation 3.5). Only one figure was included to demonstrate this result. 


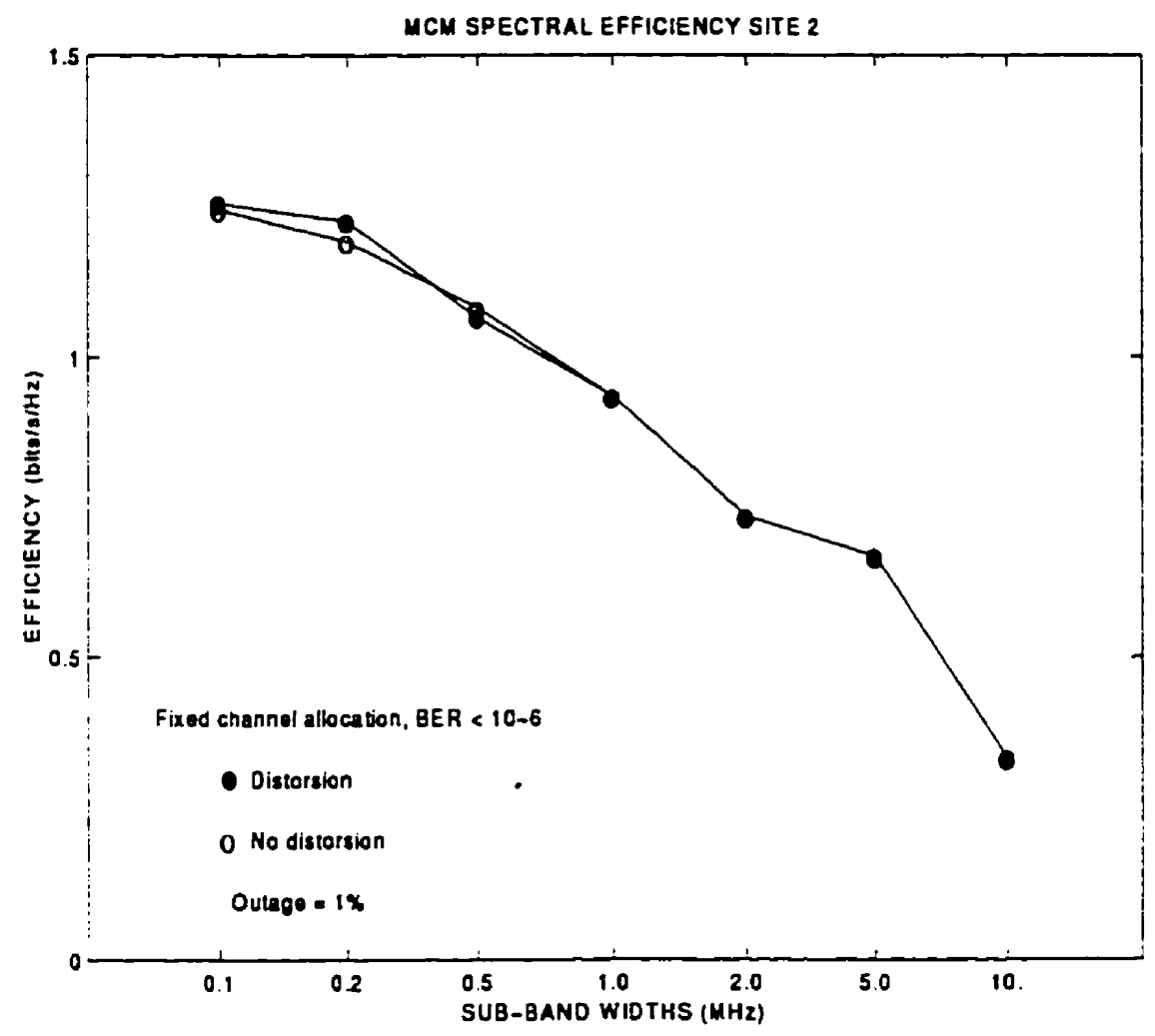

Figure 4.15: Performance of a channel with distortion and a channel without distortion foi site 2 using the MCM system. 


\subsection{Wideband system with infinite DFE (WDFE)}

\subsubsection{Effect of the sub-band width on capacity}

The same analysis as the MCM scheme is repeated here using the WDFE with an infinite number of taps in order to determine an upper bound on the performance of this scheme. In this case the infinite taps equalizer is contending with coloured ingress noise instead of channel distortion. Section 3.3 gives the details for the evaluation using the infinite DFE. The finite DFE model will be presented in section 4.5. The same sub-band widths of $.1, .2$, $.5,1,2,5$ and $10 \mathrm{MHz}$ were used to view the effect of sub-band width on channel capacity. Note that due to the spectral analyzer resolution of 100 $\mathrm{kHz}$, the $100 \mathrm{kHz}$ sub-band width will give the same results as for the MCM (refer to section 3.4.3). Moreover, for the practical implementation of the WDFE scheme, only sub-bands in the order of $\mathrm{MHz}$ should be considered. The capacity was evaluated using a FCA and VCA scheme with the parameters of $1 \%$ outage and a BER less than $10^{-6}$ and $10^{-9}$.

The graphs

All the figures in this section can be interpreted as follows: the vertical left axis represents the efficiency in bits per second per hertz $(\mathrm{bps} / \mathrm{Hz})$; the vertical right axis represents the total capacity for the portion of the spectrum under study; and the bottom horizontal axis represents each sub-band width that was used to compute the capacity. Each graph contains two curves corresponding to the BER of $10^{-6}$ and $10^{-9}$, plotting the efficiency versus the sub-band width. 
The $\diamond$ and $\Delta$ symbols have been included in the $100 \mathrm{kHz}$ sub-band to represent the MCM ferformance for the VCA and FCA scheme respectively.

\subsubsection{5-35 $\mathrm{MHz}$ portion}

Figure 4.16 and figure 4.17 show the results for site 1 and site 2 for the FCA. Figure 4.18 and figure 4.19 show the results for the VCA. Contrary to the MCM system, outage performance is improved by widening the sub-band for the FCA method, thus exploiting the non-whiteness of the noise in the larger sub-band widths. Figures 4.16 and 4.17 present better results by having wider sub-band widths. The effect of the sub-band widths is of less importance compared to the MCM system for the time varying allocation method. This can be explained by the fact that the DFE equalizer is able to mitigate the noise and that most of the sub-bands located in the higher portion of the spectrum will present the same SNR level after equalization, thus removing the need for allocating data to another sub-band.

\subsubsection{5-15 $\mathrm{MHz}$ portion}

Figure 4.20 and figure 4.21 show the results of site 1 and site 2 respectively for the lower end portion of the spectrum for the FCA scheme. Figure 4.22 and figure 4.23 show the results for the VCA scheme. This is the only portion of the return spectrum for the WDFE scheme where we have a case of better performance using smaller sub-band width. This effect has a tendency to appear in the presence of high noise energy when we use the time varying 


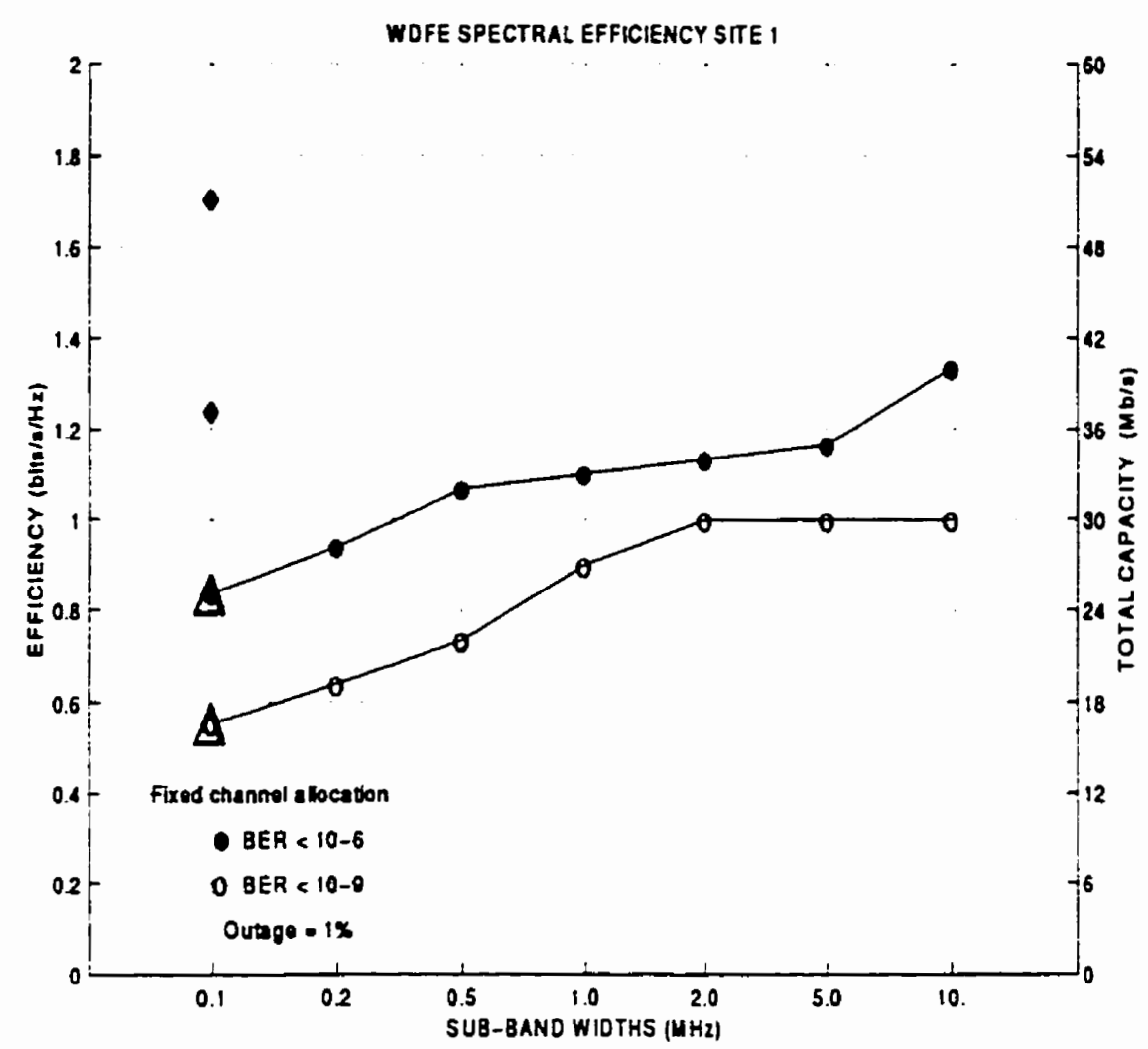

Figure 4.16: Efficiency of the 5-35 $\mathrm{MHz}$ spectrum of site 1 versus sub-channel bandwidth for a FCA scheme. 


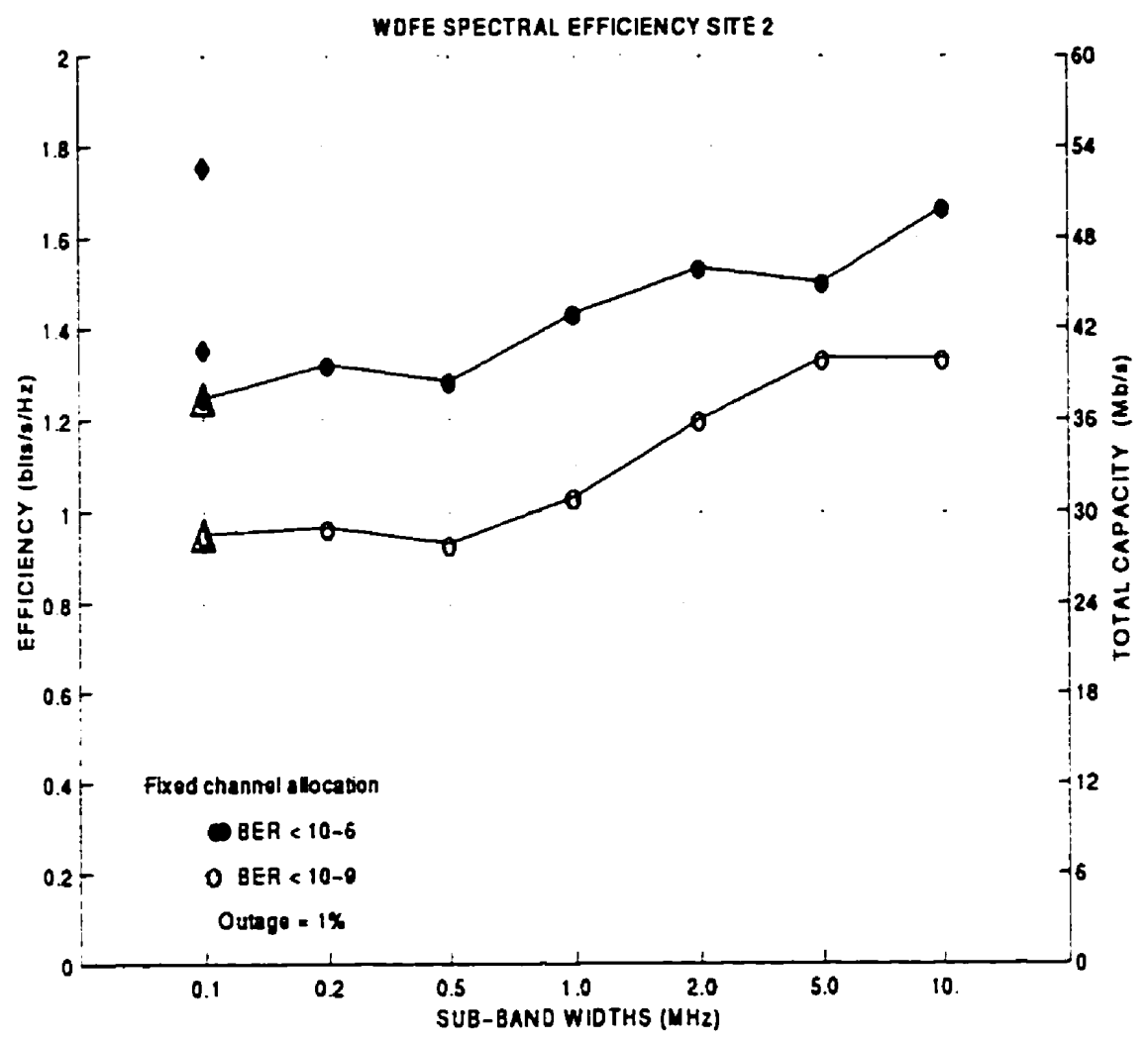

Figure 4.17: Efficiency of the 5-35 $\mathrm{MHz}$ spectrum of site 2 versus sub-channel bandwidth for a FCA scheme. 


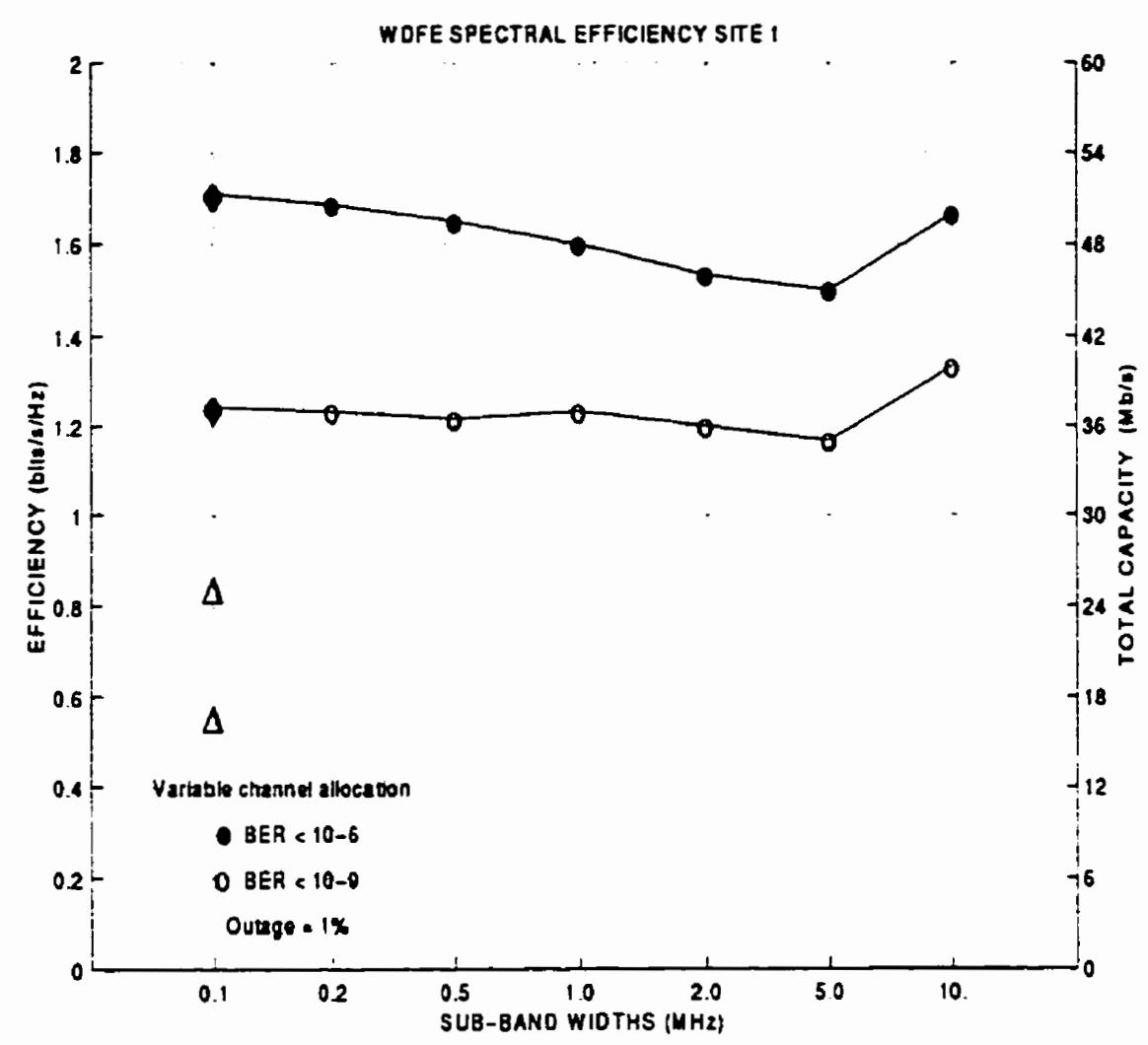

Figure 4.18: Efficiency for the 5-35 $\mathrm{MHz}$ spectrum of site 1 versus sub-channel bandwidth for a VCA scheme. 


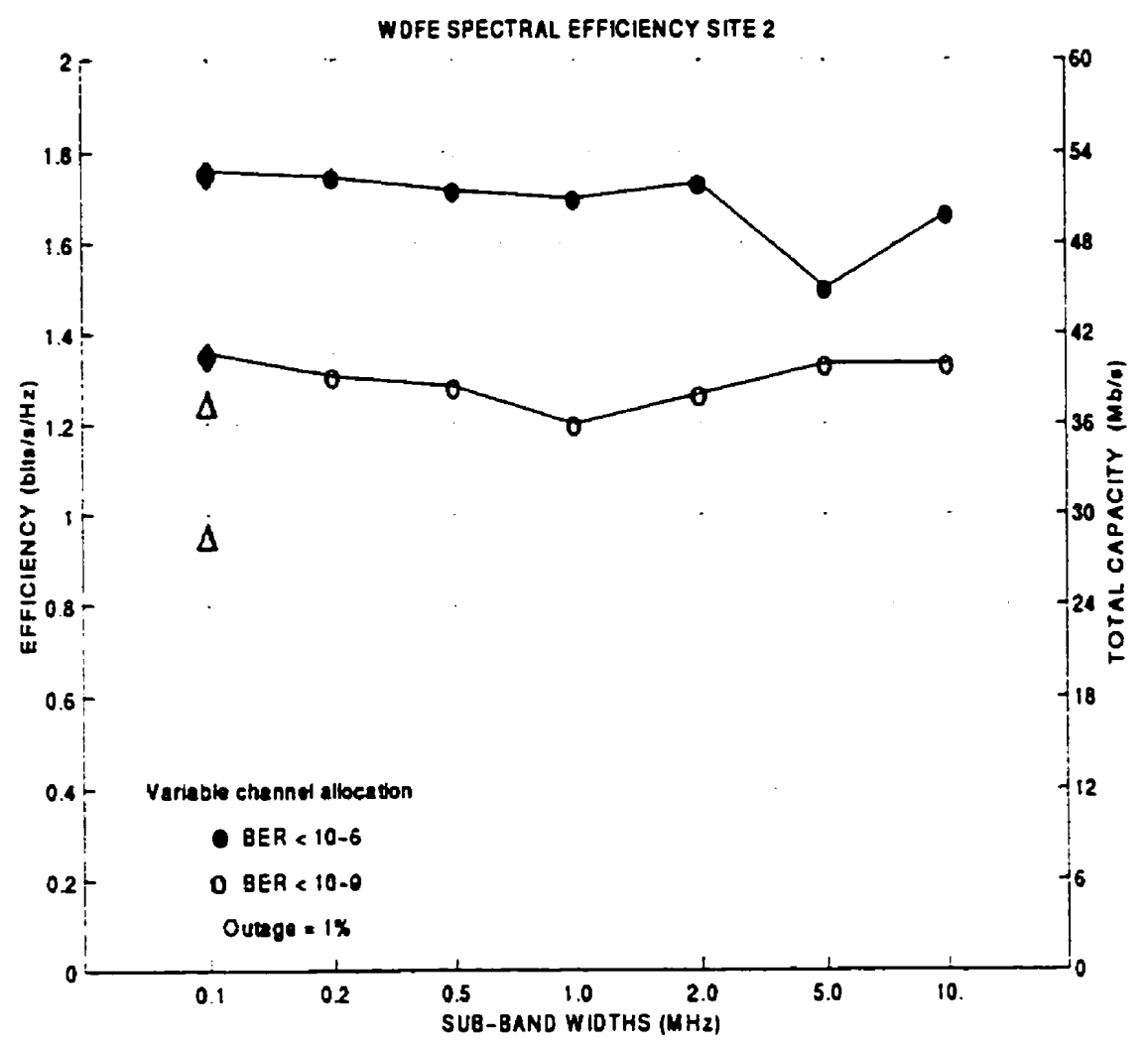

Figure 4.19: Efficiency of the 5-35 $\mathrm{MHz}$ spectrum of site 2 versus sub-channel bandwidth foi a VCA scheme. 
allocation scheme. In this case, the noise is so bad that the DFE equalizer cannot entirely mitigate the ingress noise and the usage of smaller sub-bands does improve the performance. We also have a special case in figure 4.21 and figure 4.23 where the sub-bands of 5 and $10 \mathrm{MHz}$ present irregularities in regards to the usage of smaller sub-bands. This is because the $5-15 \mathrm{MHz}$ spectrum contains only 1 sub-band of $10 \mathrm{MHz}$ wide. Since the VCA and FCA schemes both have outage requirements of $1 \%$, both schemes will present the same results, as the VCA scheme cannot allocate to another sub-band. In this particular case, the SNR level is between 10.5 and $12.5 \mathrm{~dB}$. We will therefore have an efficiency of $1 \mathrm{bps} / \mathrm{Hz}$ for the BER of $10^{-6}$ and 0 for the BER of $10^{-9}$.

\subsubsection{15-35 $\mathrm{MHz}$ portion}

Figure 4.24 and figure 4.25 show the FCA results for both sites. Figure 4.26 and figure 4.27 show the VCA results for both sites. Again, as seen in section 4.3.1.1, the use of smaller sub-band width is not required to obtain relatively good performance.

\subsubsection{FCA versus VCA for WDFE}

In this section, we compare the performance of the FCA scheme with the VCA scheme to see if a significant improvement can be obtained by using a more complex scheme such as VCA. As with the MCM systern, the same parameters and assumptions are used: both systems must not allow more 


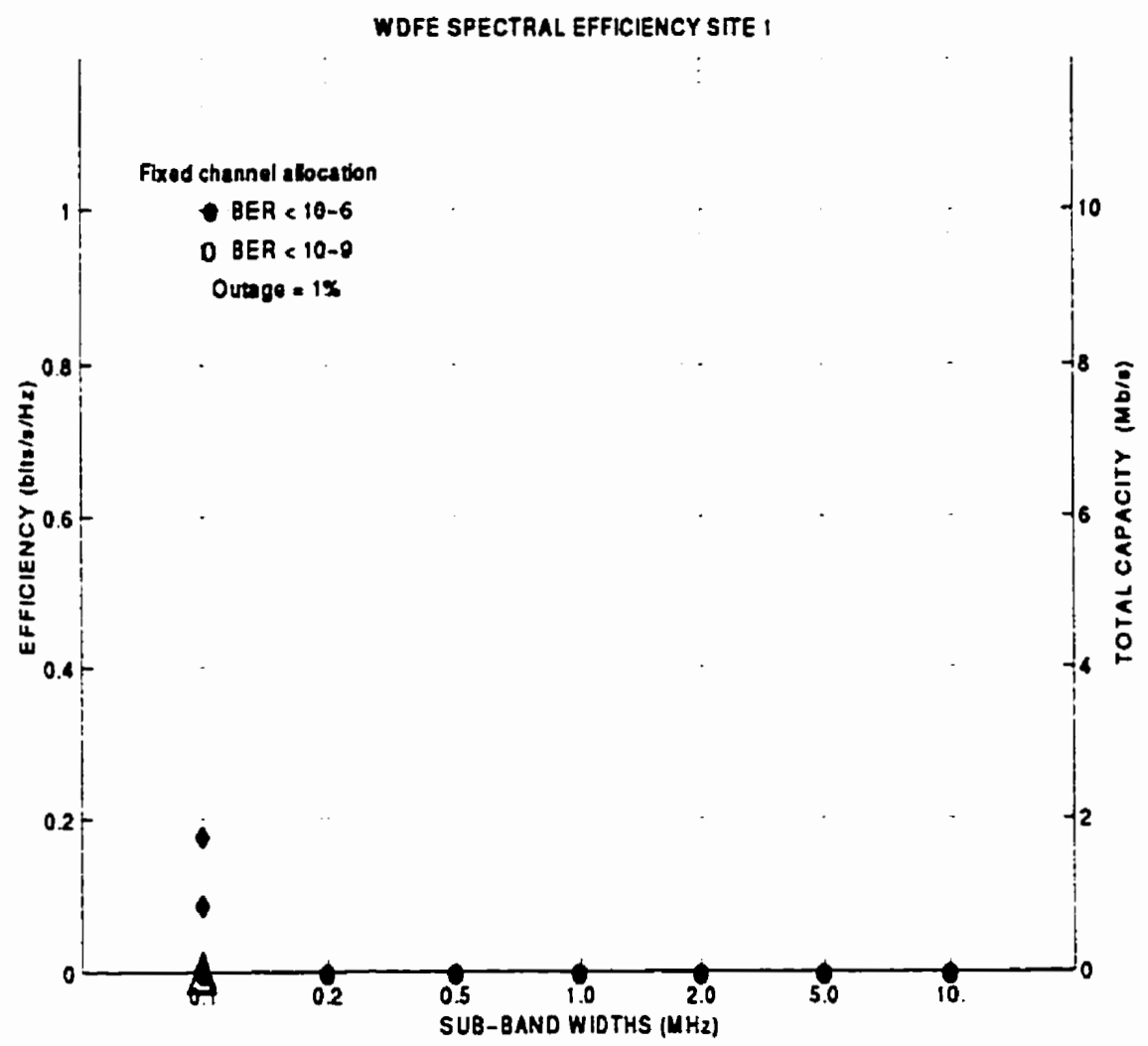

Figure 4.20: Efficiency of the 5-15 $\mathrm{MHz}$ spectrum of site 1 versus sub-channel bandwidth for a FCA scheme. 


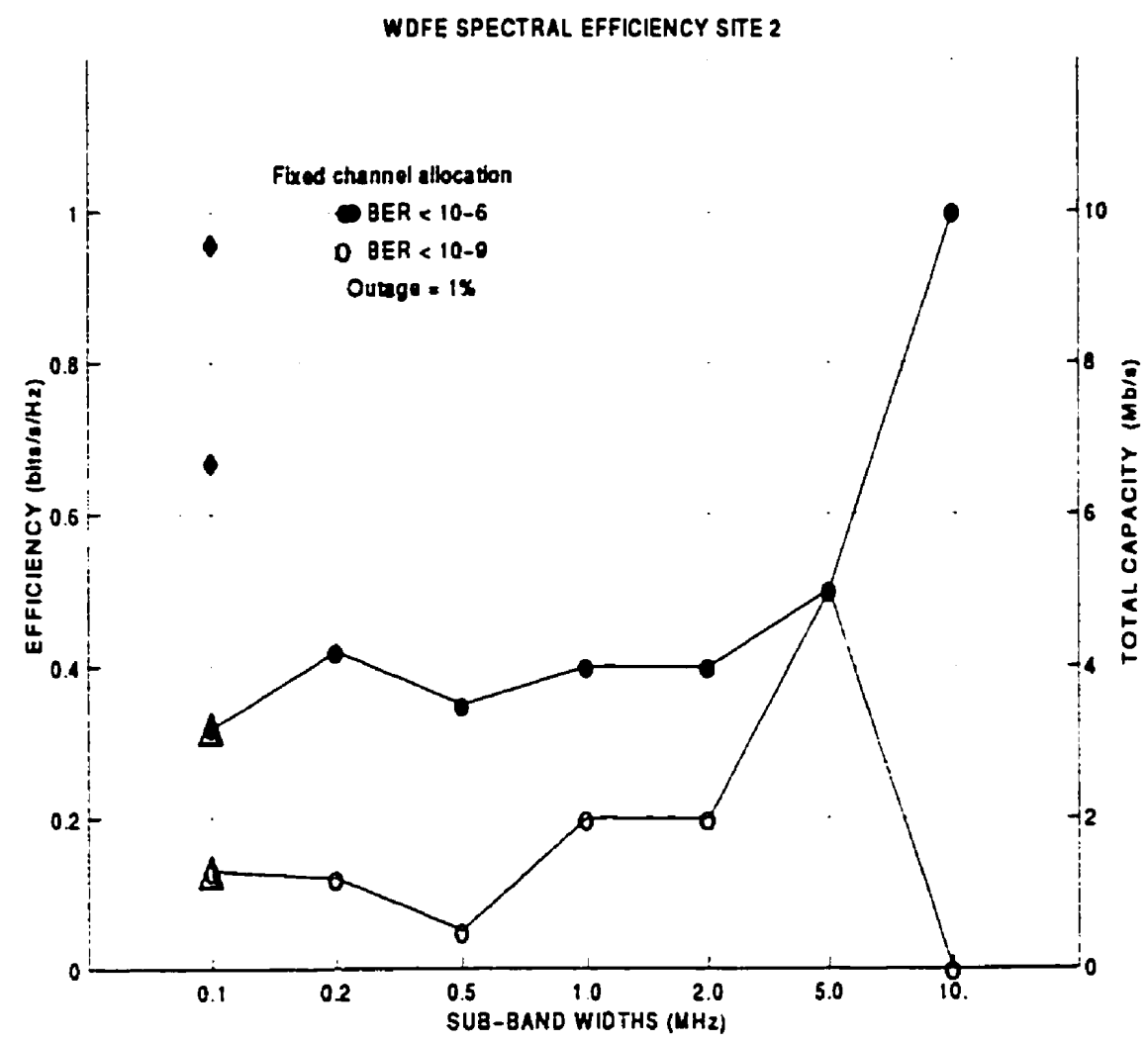

Figure 4.21: Efficiency of the 5-15 MHz spectrum of site 2 versus sub-channel bandwidth for a FCA scheme. 


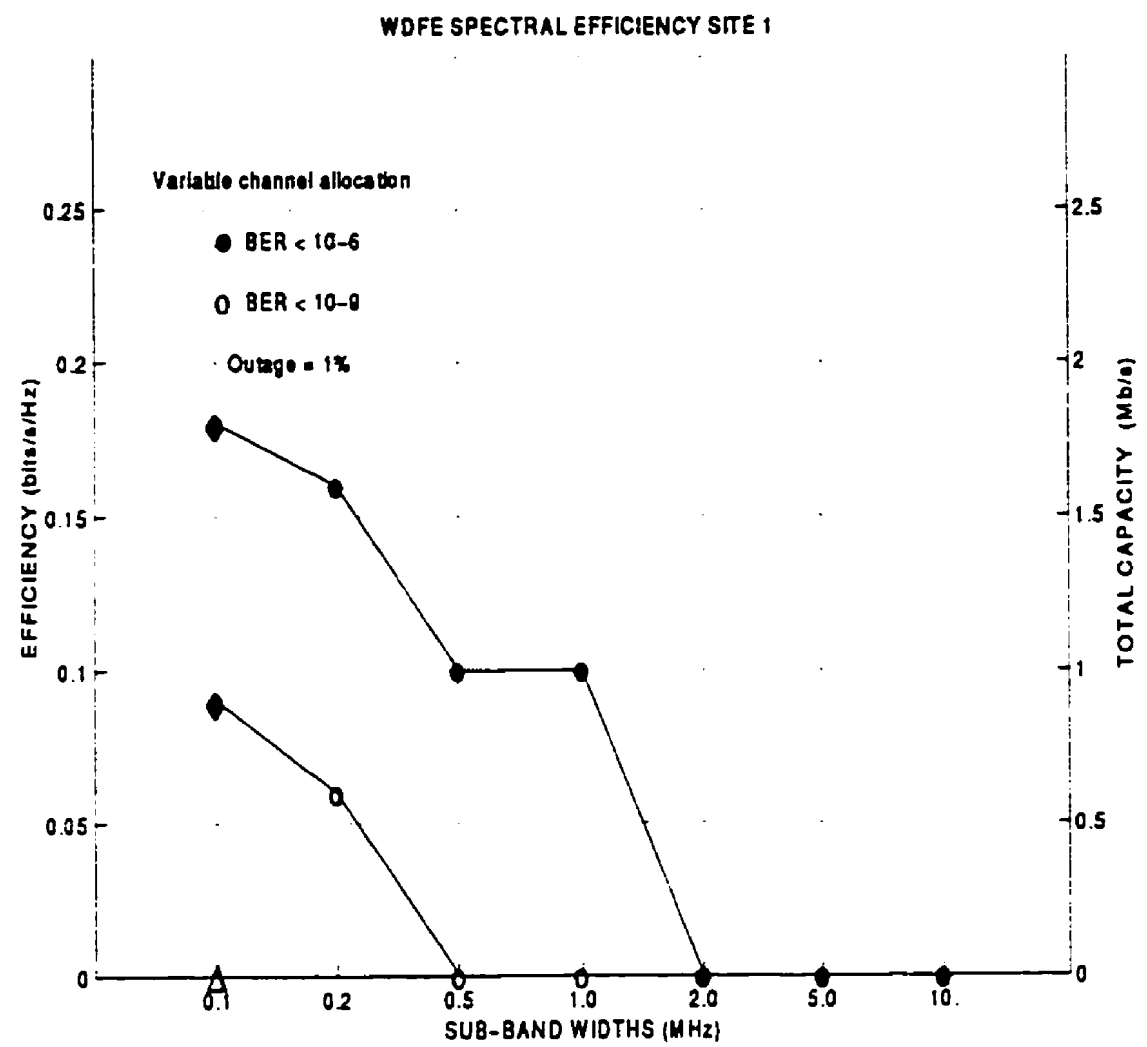

Figure 4.22: Efficiency of the 5-15 MHz spectrum of site 1 versus sub-channel bandwidth for a VCA scheme. 


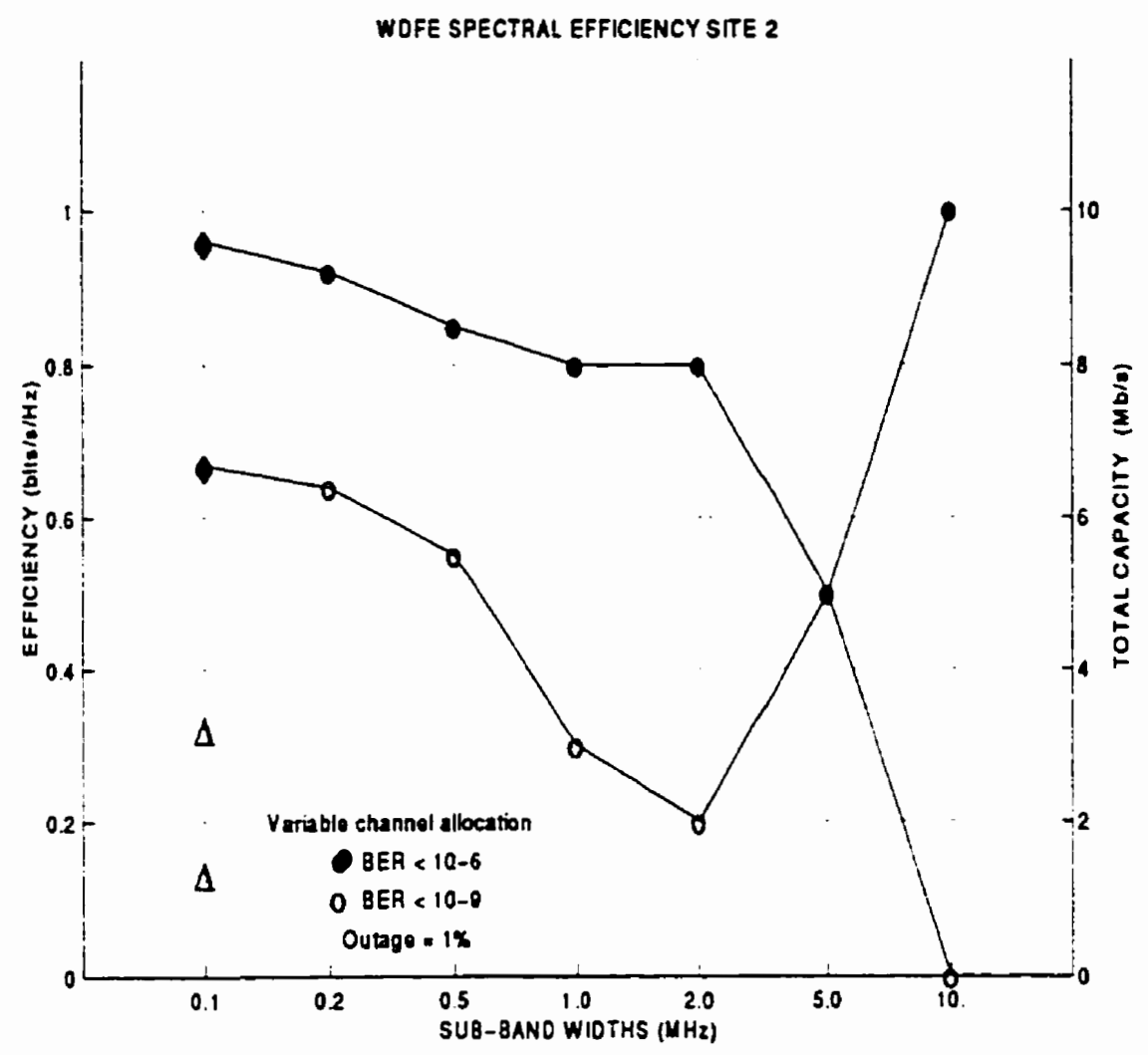

Figure 4.23: Efficiency of the 5-15 $\mathrm{MHz}$ spectrum of site 2 versus sub-channel bandwidth for a VCA scheme. 


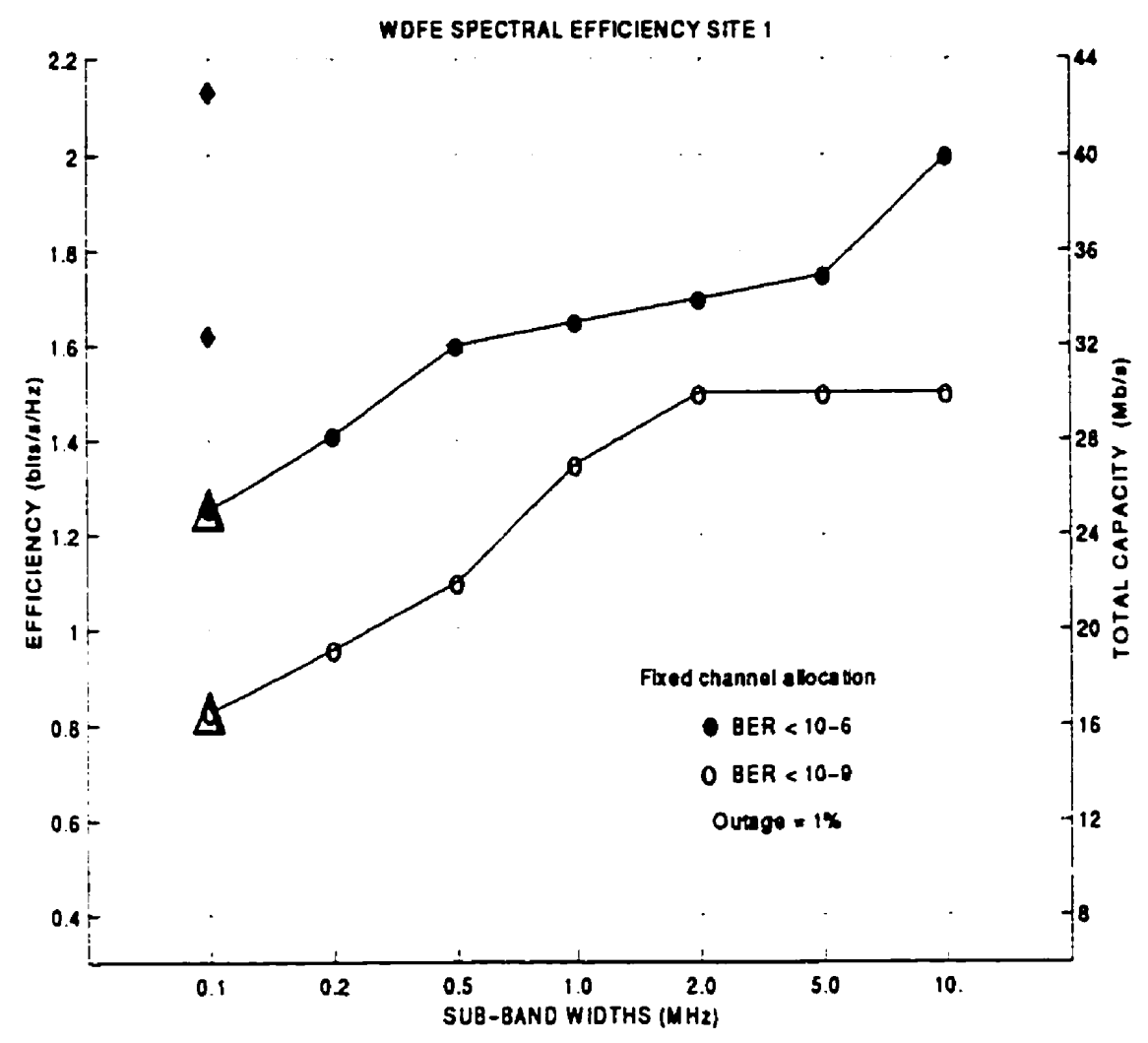

Figure 4.24: Efficiency of the $15-35 \mathrm{MHz}$ spectrum of site 1 versus sub-channel bandwidth fo: a FCA scheme. 


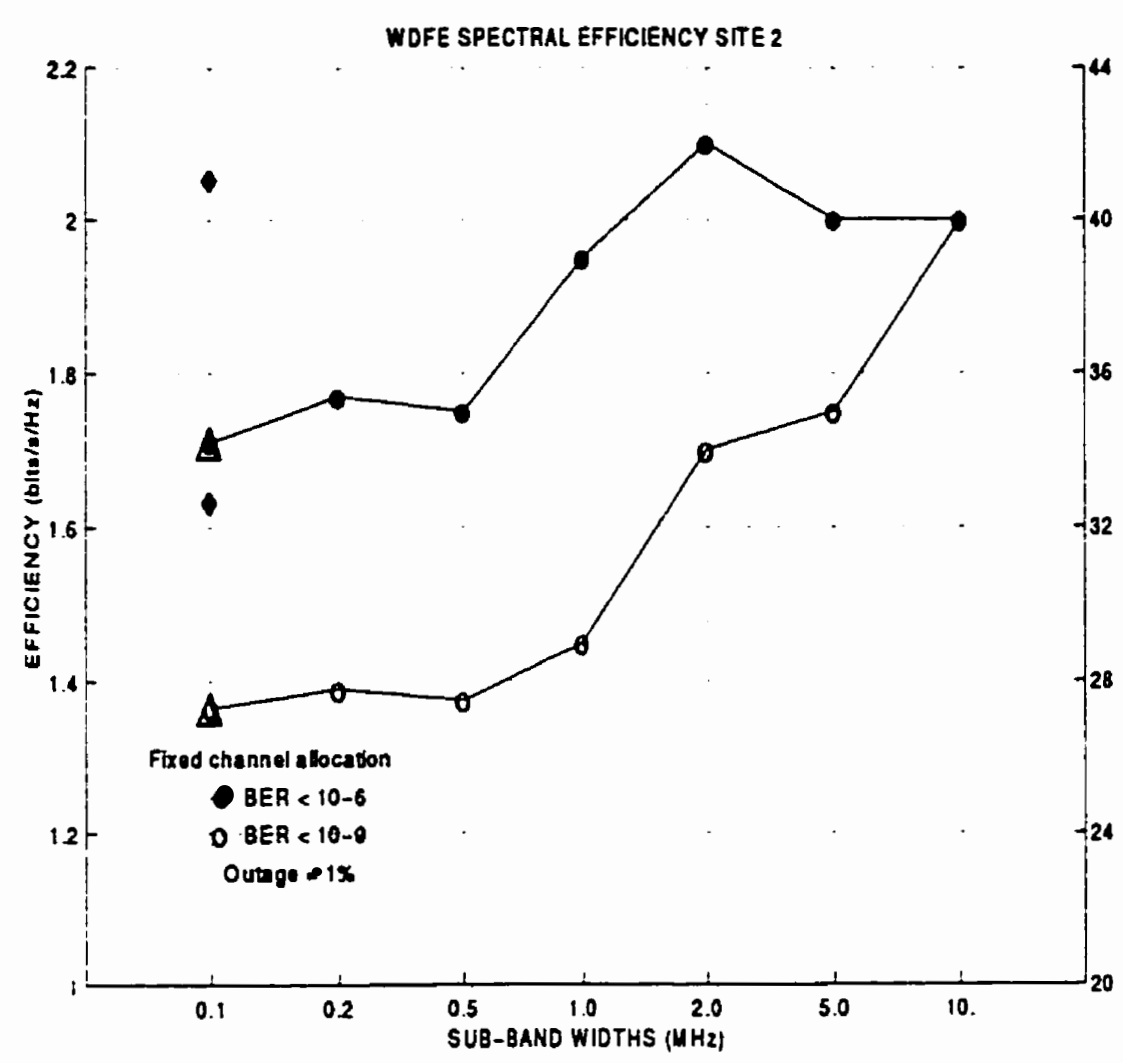

Figure 4.25: Efficiency of the 15-35 $\mathrm{MHz}$ spectrum of site 2 versus sub-channel bandwidth for a FCA scheme. 


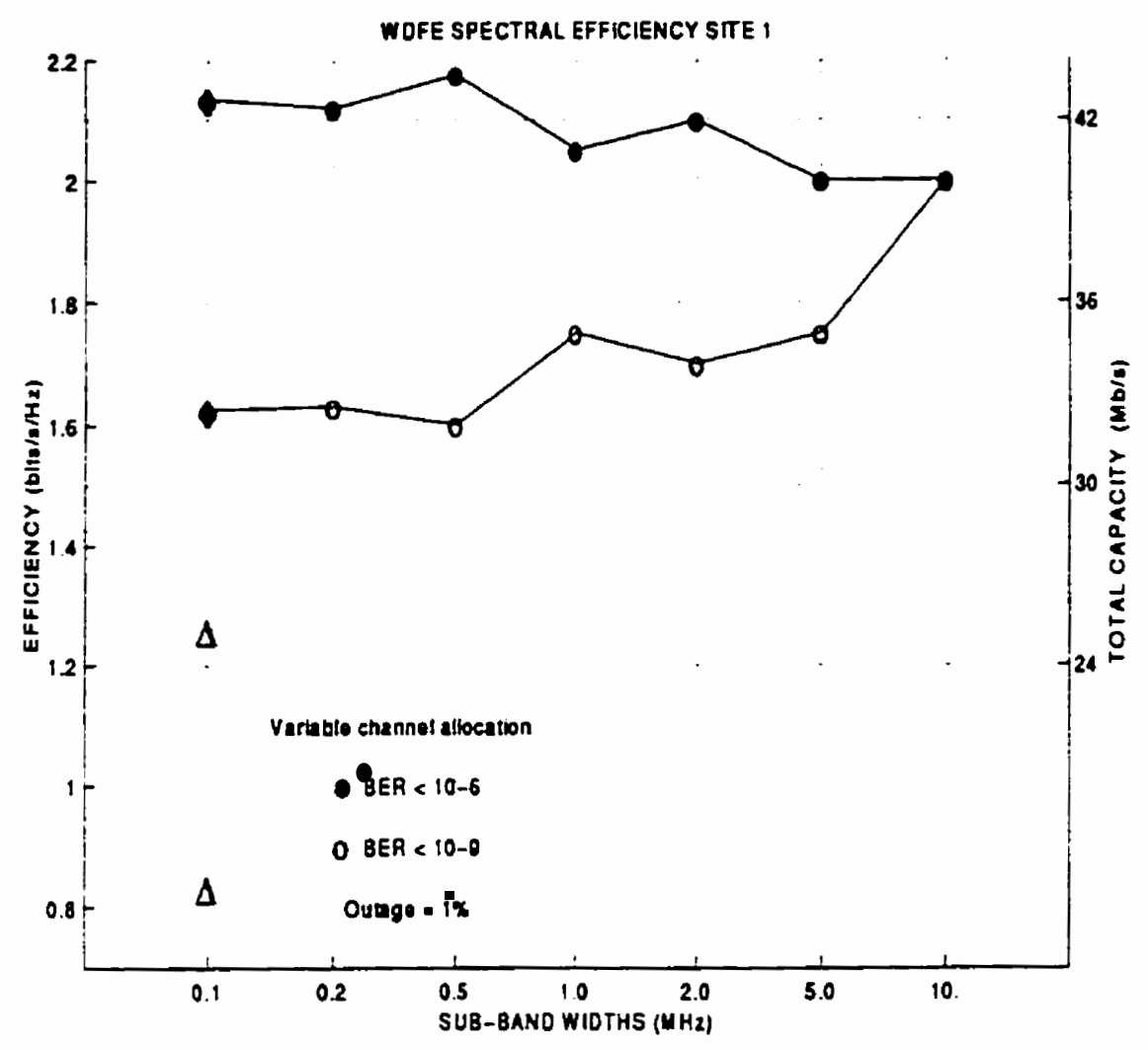

Figure 4.26: Efficiency of the 15-35 MHz spectrum of site 1 versus sub-channel bandwidth for a VCA scheme. 


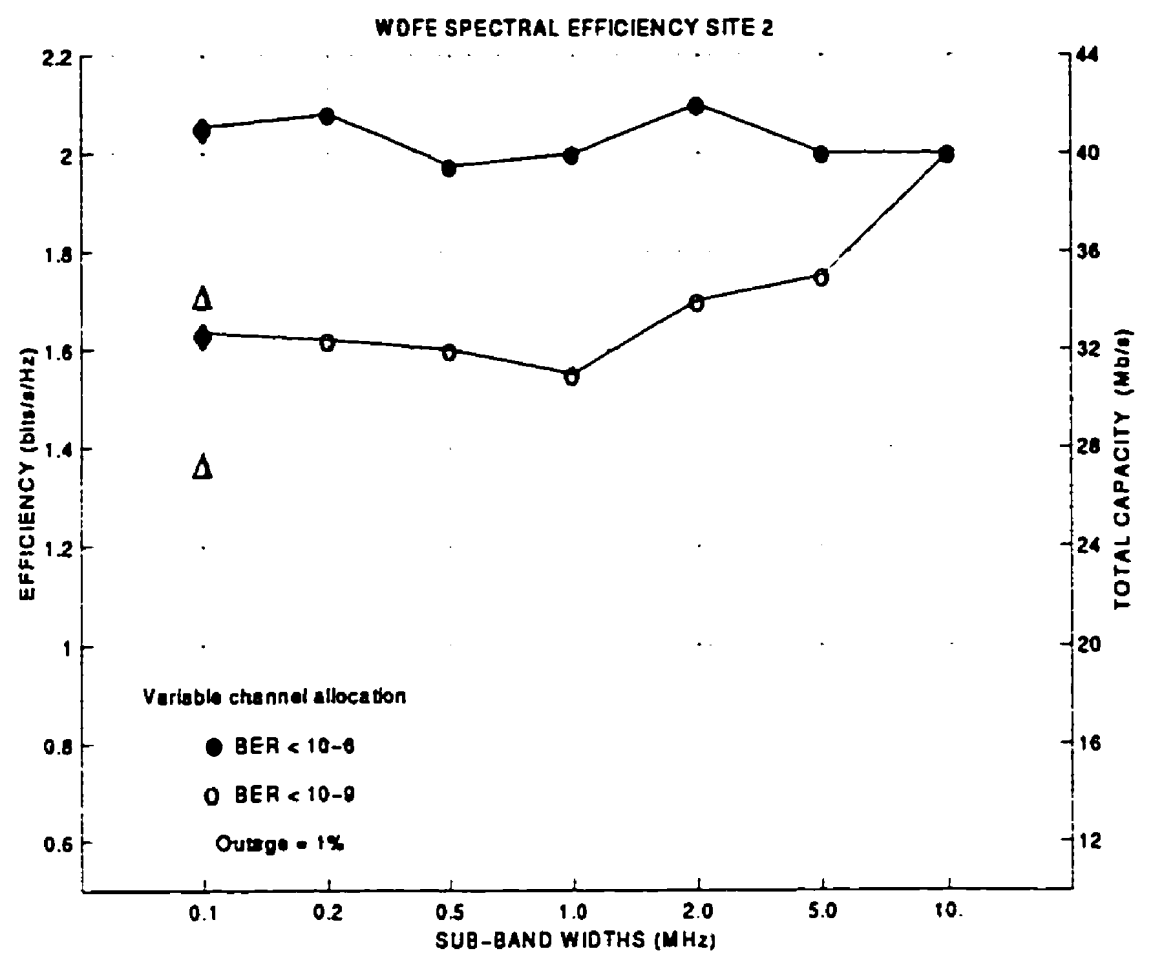

Figure 4.27: Efficiency of the $15-35 \mathrm{MHz}$ spectrum of site 2 versus sub-channel bandwidth for a VCA scheme. 
than $1 \%$ outage, the VCA method is able to effectively adapt its modulation scheme on a minute to minute basis, and we are dealing with slowly time varying ingress noise. We can see by comparing the figures $4.16,4.17,4.20$, $4.21,4.24$ and 4.25 with figures $4.18,4.19,4.22,4.23,4.26$ and 4.27 that all portious of the spectrum show less improvement compared to the results found with the MCM scheme in section 4.2.2. Table 4.2 presents the improvement in efficiency for both sites for the sub-band width of $1 \mathrm{MHz}$. Note that the time varying allocation is also more effective in the lower end with the presence of more noise energy compared to the higher portion of the spectrum. As was mentioned in the previous section, this can be explained by the fact that the WDFE cannot entirely mitigate the ingress noise so the effect of using smaller sub-bands starts to prevail. In contrast, for the higher portion of the spectrum, there is less difference between the VCA and FCA methods for the WDFE compared to the MCM. This is even more evident with site 2, where there is only $3 \%$ improvement of VCA over FCA.

Table 4.2: Performance of VCA over FCA.

\begin{tabular}{|c|c|c|c|}
\hline Site & $5-35 \mathrm{MHz}$ & $5-15 \mathrm{MHz}$ & $15-35 \mathrm{MHz}$ \\
\hline \hline 1 & $39 \%$ & $\infty$ & $25 \%$ \\
\hline 2 & $21 \%$ & $100 \%$ & $3 \%$ \\
\hline
\end{tabular}




\subsubsection{Channel distortion by reflections}

Figure 4.28 presents the results for a channel with and without distortion. The same two taps channel model was used as in the MCM scheme. We can assess that the distortion has almost no effect on the results. This is not suprising, since we have previously obtained this result for the MCM case. Moreover, the infinite taps DFE is able to cancel out part of the distortion caused by the reflections.

\subsection{MCM versus WDFE}

In this section, the performance of both systems for all portions of the spectrum is compared. The following tables outline the performance of each system. Note that to make this comparison, we used $100 \mathrm{kHz}$ sub-band width for the MCM system and $2 \mathrm{MHz}$ sub-band width for the WDFE system. The performance values shown in tables 4.3 and 4.4 correspond to the performance improvement of the MCM system over the WDFE system. A negative value therefore means that the WDFE system was better in that case. The results were also computed for the FCA and VCA scheme.

We can see that for the FCA scheme, the WDFE system presents better performance, even in the low portion of the spectrum.

For the VCA scheme, we see that both systems present similar results. The only case where the MCM has significant advantage is in the low portion 


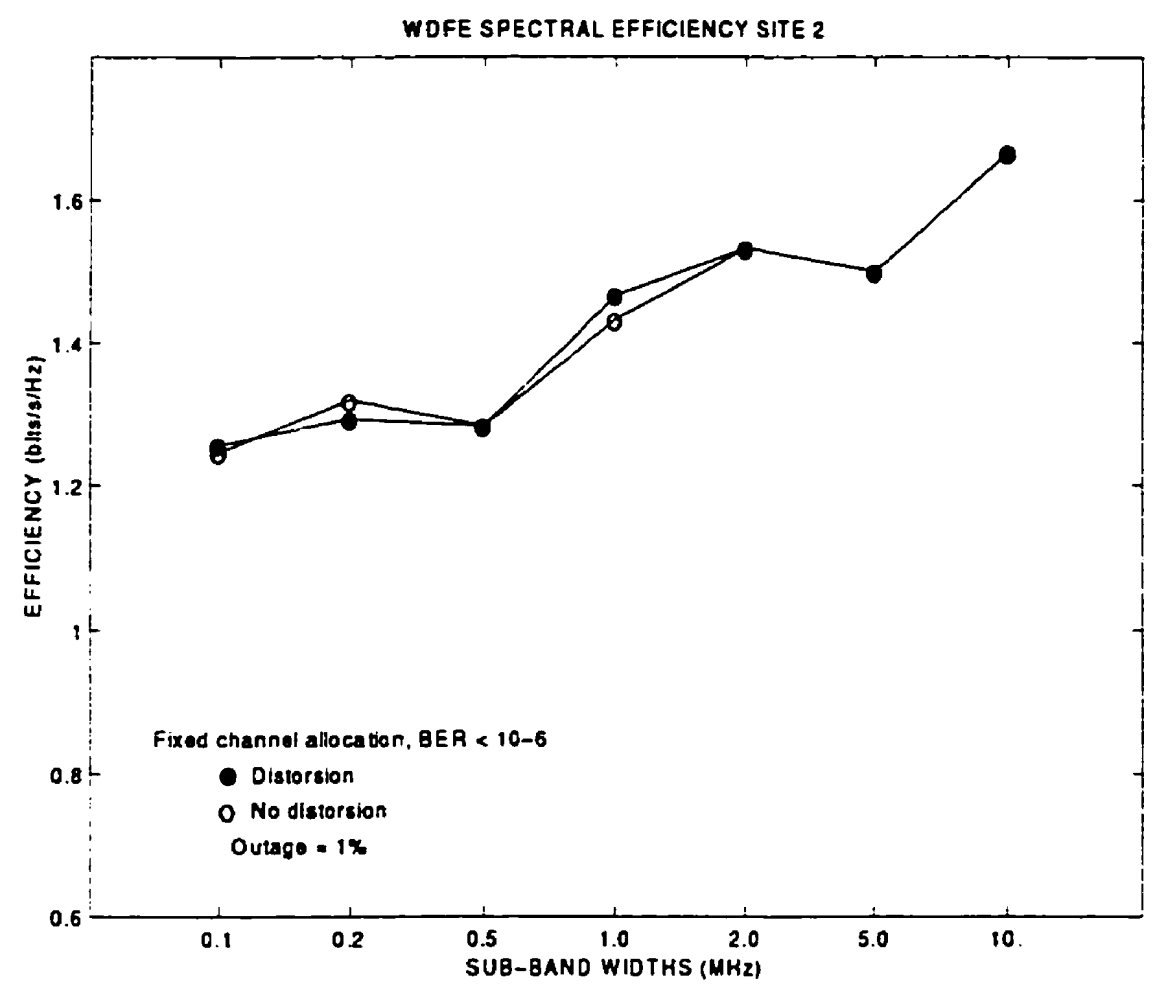

Figure 4.28: Performance of a channel with distortion and a channel without distortion for site 2 using the WDFE with an infinite number of taps. 
Table 4.3: Fixed channel allocation.

\begin{tabular}{|c|c|c|c|}
\hline Site & $5-35 \mathrm{MHz}$ & $5-15 \mathrm{MHz}$ & $15-35 \mathrm{MHz}$ \\
\hline \hline 1 & $-35 \%$ & $\infty$ & $-35 \%$ \\
\hline 2 & $-23 \%$ & $-25 \%$ & $-23 \%$ \\
\hline
\end{tabular}

Table 4.4: Variable channel allocation.

\begin{tabular}{|c|c|c|c|}
\hline Site & $5-35 \mathrm{MHz}$ & $5-15 \mathrm{MHz}$ & $15-35 \mathrm{MHz}$ \\
\hline \hline 1 & $10 \%$ & $100 \%$ & $2 \%$ \\
\hline 2 & $2 \%$ & $17 \%$ & $-2 \%$ \\
\hline
\end{tabular}

of the spectrum with the presence of low SNR. Since the low portion of the spectrum is nearly unusable, both schemes give almost the same performance for the total spectrum. These results are not suprising, since prior research has shown that MCM and a wideband system using a DFE present similar results for high SNR [16]. In the high portion of the spectrum, the WDFE is very effective in mitigating the ingress noise and presents good results.

\subsubsection{WDFE using FCA versus MCM with VCA method}

It has already been shown that the WDFE system has superior performance over MCM for the FCA scheme. The complexity of designing the time varying allocation scheme, where the modem has to be able to reduce its efficiency in order to adapt itself to the time varying aspect of the noise, is important. 
It is interesting to compare the FCA method using the WDFE system with the VCA method using the MCM system to see if it is worthwhile to build a system of such complexity. The following table compares the channel capacity of these two approaches. We can assess that the WDFE - FCA could approach the capacity of MCM - VCA without the protocol complexity inherent in the VCA scheme.

Table 4.5: Improvement in efficiency of the MCM - VCA over the WDFE FCA for $2 \mathrm{MHz}$ sub-bands.

\begin{tabular}{|c|c|c|c|}
\hline Site & $5-35 \mathrm{MHz}$ & $5-15 \mathrm{MHz}$ & $15-35 \mathrm{MHz}$ \\
\hline \hline 1 & $34 \%$ & $100 \%$ & $20 \%$ \\
\hline 2 & $13 \%$ & $58 \%$ & $2 \%$ \\
\hline
\end{tabular}




\subsection{Wideband system with finite DFE (WFDFE)}

The model used in this section was previously described in section 3.4. The objective of this model is to determine the performance and requirements for a system representative of one encountered in practice. Note that the computation involving the efficiency has been calculated with the average throughput of the VCA scheme to better distinguish the effect of the parameters being analyzed. Most of the analysis in this section was done considering $2.1 \mathrm{MHz}$ sub-bands, which would be a realistic value for a system encountered in practice. Note also that since both sites have always shown the same tendencies to date, most of the results shown in the next sections will be for site 2 and for the BER of $10^{-6}$. In section 4.5.5, where we compare the performance of the WFDFE versus other models, we have included the results for both sites and for the sub-bands of 2.1 and $10.1 \mathrm{MHz}$.

\subsubsection{Number of taps}

The tap requirements have been computed considering the distorted channel and synchronous equalization. The case for adjacent channel interference will be further discussed in section 4.5.4 with fractionally spaced equalization.

\section{The graphs}

The figures in this section may be interpreted as follows: the top and bottom graphs show the results for two sub-bands of $2 \mathrm{MHz}$ located in the low and high portion of the spectrum respectively; the symbols $\bullet, \circ$, and $\Delta$ represent 
the SNR obtained using the number of feedback taps (FB) corresponding to the integer value of forward taps (N) of $0, N / 4$ and $N / 2$ rounded down to the next lowest integer. The $\diamond$ symbol represents the bound obtained by using an infinite DFE.

Figure 4.29 shows the tap requirements for 1 sample file of site 2 . The first sub-band is located close to the 5-7 MHz portion of the spectrum while the second one is located close to the $33-35 \mathrm{MHz}$ portion. We can assess that after 10-15 taps we have reached most of the improvement obtainable by decision feedback equalization. We also see that we need more taps in the lower portion of the spectrum to reach an acceptable performance. The number of required feedback taps always reaches its maximum efficiency at approximately half the number of forward taps. This is due to the fact that the impulse response used to compute the MMSE was always minimum when the main ray was centered at the middle of the impulse response (we are not dealing with a non-minimal phase situation, where the second ray would be bigger than the first one). Moreover, the reflection is $-20 \mathrm{db}$ down from the main ray. If we place the reflection on the right of the main ray, a small improvement can be seen by having more feedback taps. This is due to the fact that part of the ISI caused by the reflection can be removed by past decisions and the forward section of the equalizer can more effectively mitigate the ingress noise. In our analysis this improvement was negligible when the number of feedback taps was at approximately half the number of forward taps.

Figure 4.30 shows the result for $10.1 \mathrm{MHz}$ sub-bands. The top and bottom 
correspond to the results for two sub-bands located in the low and high portion of the spectrum respectively. We can see that we need more taps when we use larger sub-bands. This is a natural consequence of the bit rate increasing with the bandwidth. Notice also that for both sub-band widths, the convergence towards the infinite DFE bound is obtained more rapidly in the higher portion of the spectrum. This can be explained by the higher noise energy present in the lower portion of the spectrum. Further analysis regarding the number of taps will be done in section 4.5.3, where we will view the effect of various numbers of taps and carrier spacing on efficiency.

\subsubsection{The effect of the excess bandwith parameter}

Only a small improvement was obtained by using fixed transmitting and receiving filters with higher excess bandwidth, and by assuming no adjacent channel interference. Table 4.6 shows the results of efficiency for site 2 . Again, only a small improvement can be observed due to the fact that we are dealing with almost an ideal channel. Note also that in this analysis, we assume perfect symbol timing and perfect carrier synchronization. In the cases where those assumptions would not hold, the usage of excess bandwidth might improve the performance of the system. Note that these results are for synchronous equalizers. 

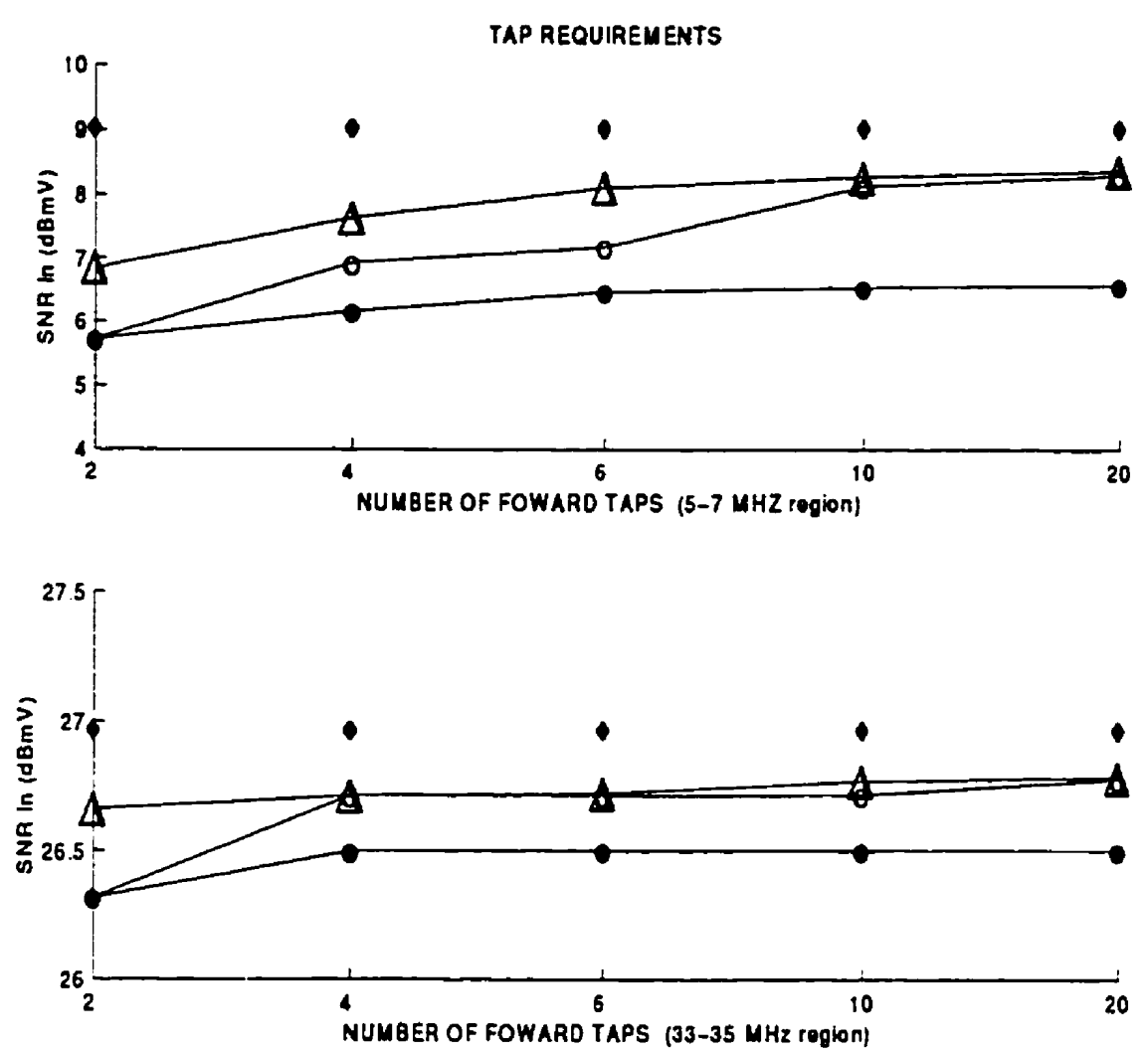

Figure 4.29: Taps requirements (2.1 $\mathrm{MHz}$ sub-bands, site 2). 


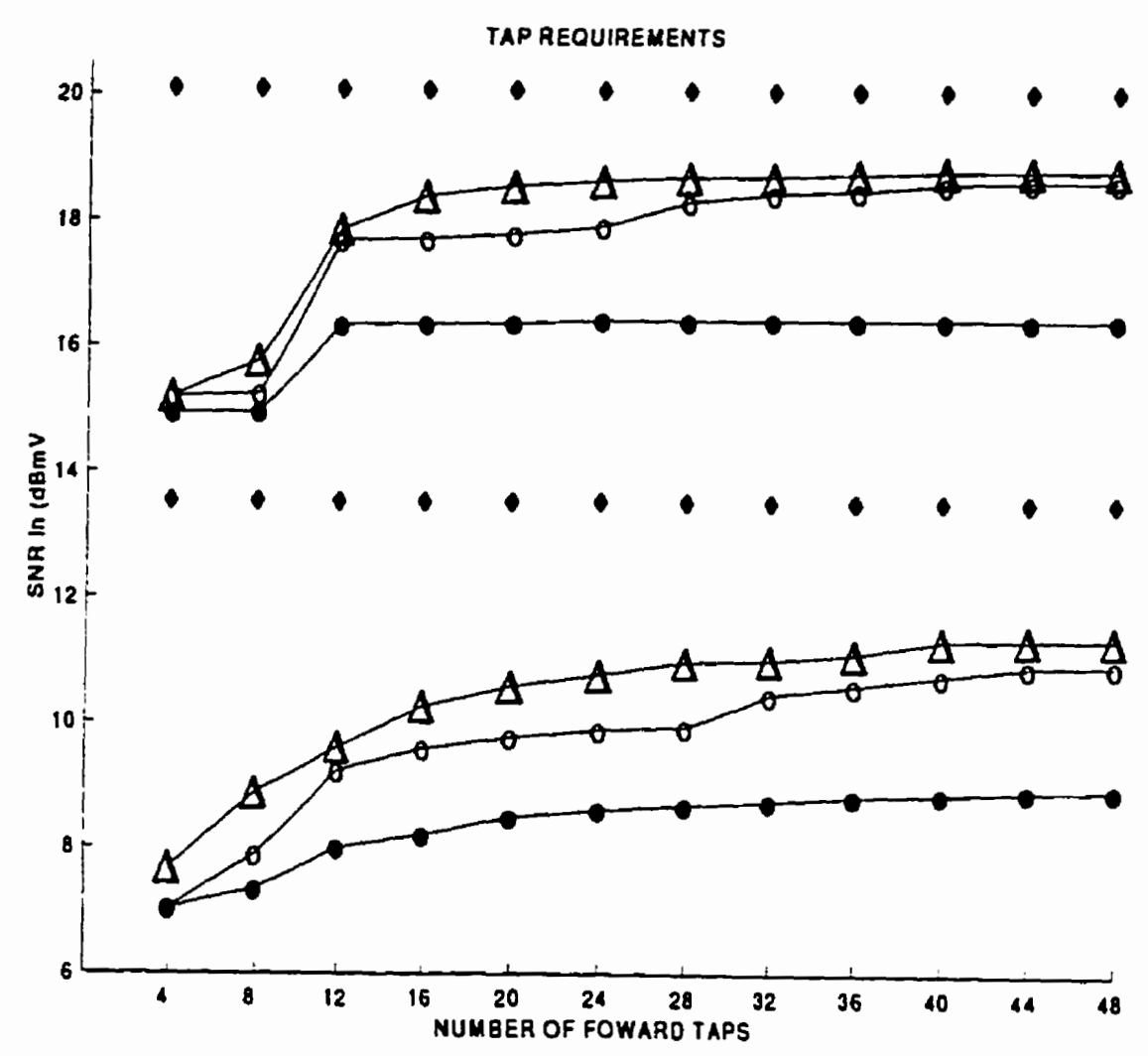

Figure 4.30: Taps requirements (10.1 MHz sub-bands, site 2). 
Table 4.6: Average efficiency (VCA - AVG) as defined in section 3.2.4 versus excess bandwidth for the low and high portions of the spectrum with $2.1 \mathrm{MHz}$ sub-bands and synchronous equalization.

\begin{tabular}{|c|c|c|c|}
\hline Spectrum (MHz) & Taps N/FB & Eff. $(\alpha=25 \%) \mathrm{b} / \mathrm{s} / \mathrm{Hz}$ & Eff. $(\alpha=10 \%) \mathrm{b} / \mathrm{s} / \mathrm{Hz}$ \\
\hline \hline Low (5-15) & $6 / 3$ & 1.38 & 1.36 \\
\hline Low (5-15) & $10 / 5$ & 1.43 & 1.40 \\
\hline Low (5-15) & $20 / 10$ & 1.45 & 1.42 \\
\hline High (15-35) & $6 / 3$ & 3.16 & 3.13 \\
\hline High (15-35) & $10 / 5$ & 3.21 & 3.17 \\
\hline High (15-35) & $20 / 10$ & 3.22 & 3.19 \\
\hline
\end{tabular}




\subsubsection{Effect of guardbands in adjacent channel interfer- ence}

An interesting fact about the decision feedback equalizer is that it can be used to suppress adjacent channel interference when we use carriers that are spaced in such way that their individual spectra overlap [13], [24].

In this section, we will give the average efficiency considering various carrier spacings for the synchronous DFE. The effect of using fractionally spaced equalization will be discussed in section 4.5.4.

The graphs

Three curves are shown representing the taps configuration of $20 / 10,10 / 5$ and $6 / 3$ forward/feedback taps. Each curve plots the average efficiency versus the overlapping factor of $0 \%, 5 \%, 10 \%, 20 \%$ and $30 \%$ (refer to section 3.4.2.3 for the definition of overlapping factor). The results shown have been computed assuming the VCA scheme with the BER of $10^{-6}$ and $2 \mathrm{MHz}$ sub-bands.

Figures 4.31 and 4.32 present the performance for sub-bands with excess bandwidth of $25 \%$ and $10 \%$ respectively for the low portion of the spectrum. In the case of $25 \%$ excess bandwidth, we can see an improvement in capacity for an overlapping factor of $10 \%$. In the case of $10 \%$ excess bandwidth, we have an improvement for an overlapping factor of $5 \%$. We can therefore effectively improve the performance by using carriers spaced in such a way that their individual spectra overlap. The DFE is able to mitigate the adjacent channel interference. Note that we obtain a peak at $5 \%$ (instead of $10 \%$ ) for the $10 \%$ 
excess bandwidth case since we are now using sharper filters for the sub-bands.

Figures 4.33 and 4.34 demonstrate the performance for the excess bandwidth parameters of $25 \%$ and $10 \%$ respectively for the high portion of the spectrum. We observe similar results for both parameters. The only difference is that we obtain higher efficiency since we are now evaluating the higher portion of the spectrum.

We can also see the effect of the number of taps by comparing the low portion of the spectrum (higher noise energy) with the high portion of the spectrum (lower noise energy). Less improvement is seen when going from $10 / 5$ to $20 / 10$ (forward/feedback) taps for the high portion of the spectrum. 


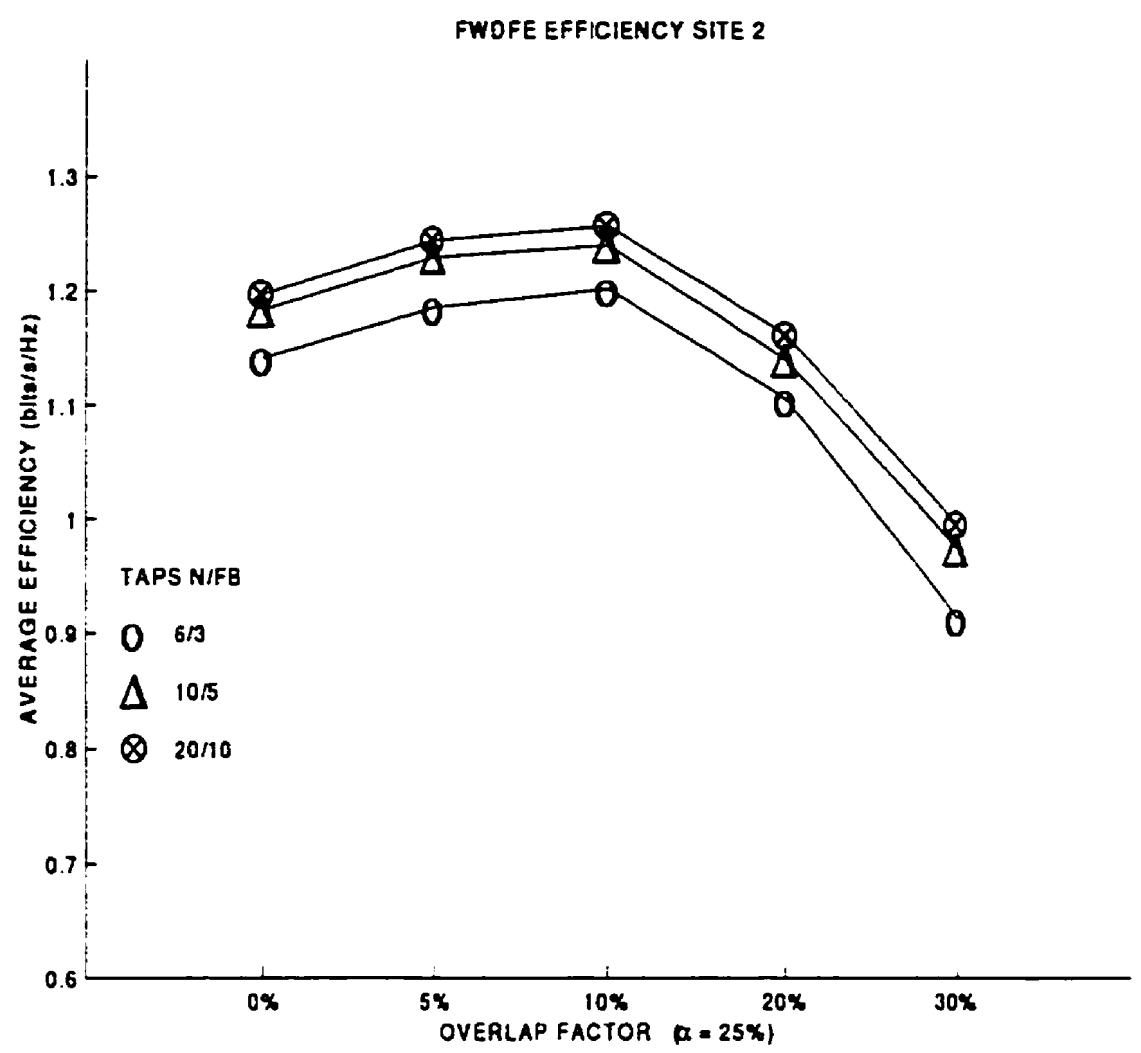

Figure 4.31: System efficiency for the low portion of the spectrum (5-15 MHz) versus overlapping factor for an equivalent raised cosine filter with $25 \%$ excess bandwidth and $2.1 \mathrm{MHz}$ sub-bands. 


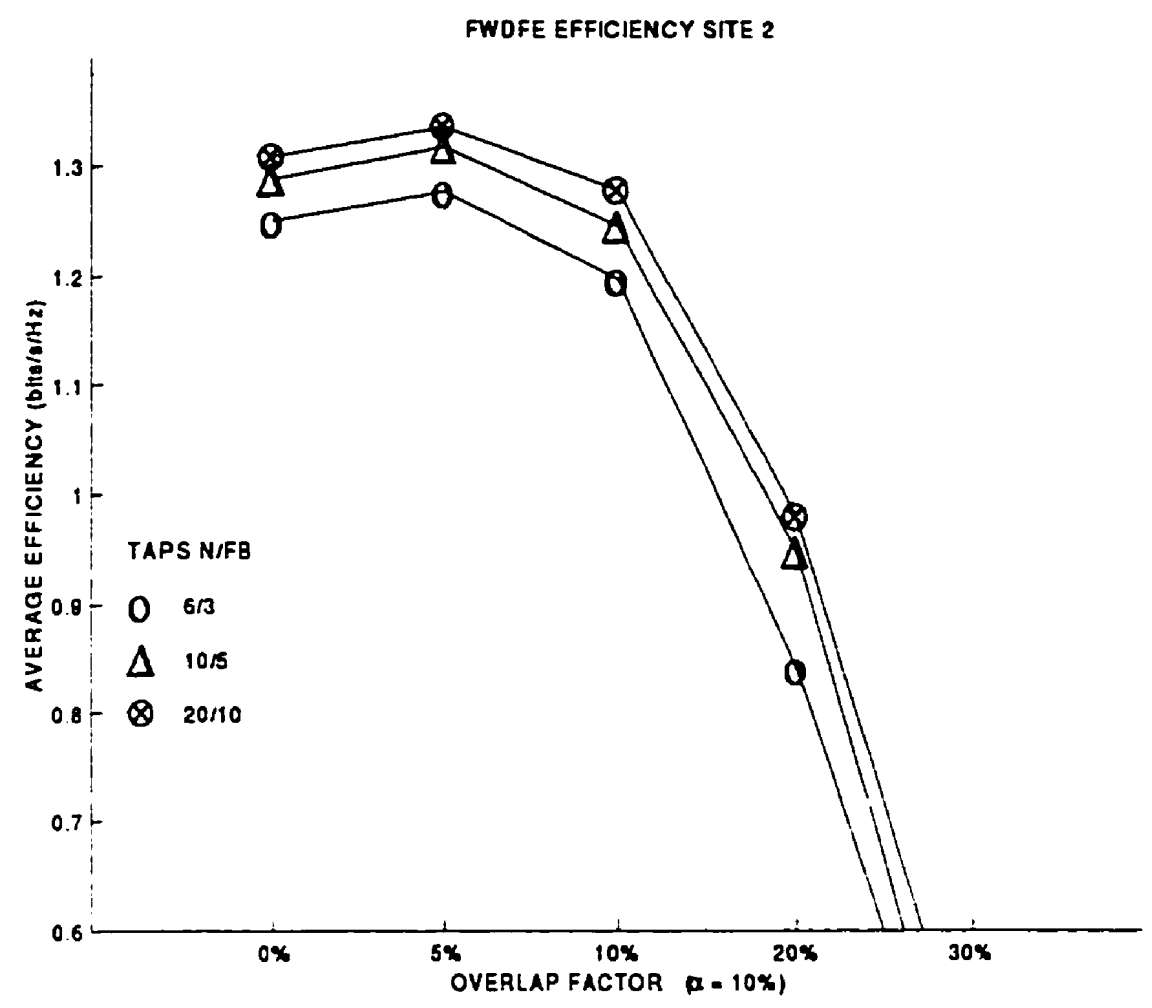

Figure 4.32: System efficiency for the low portion of the spectrum (5-15 MHz) versus overlapping factor for an equivalent raised cosine filter with $10 \%$ excess bandwidth and $2.1 \mathrm{MHz}$ sub-bands. 


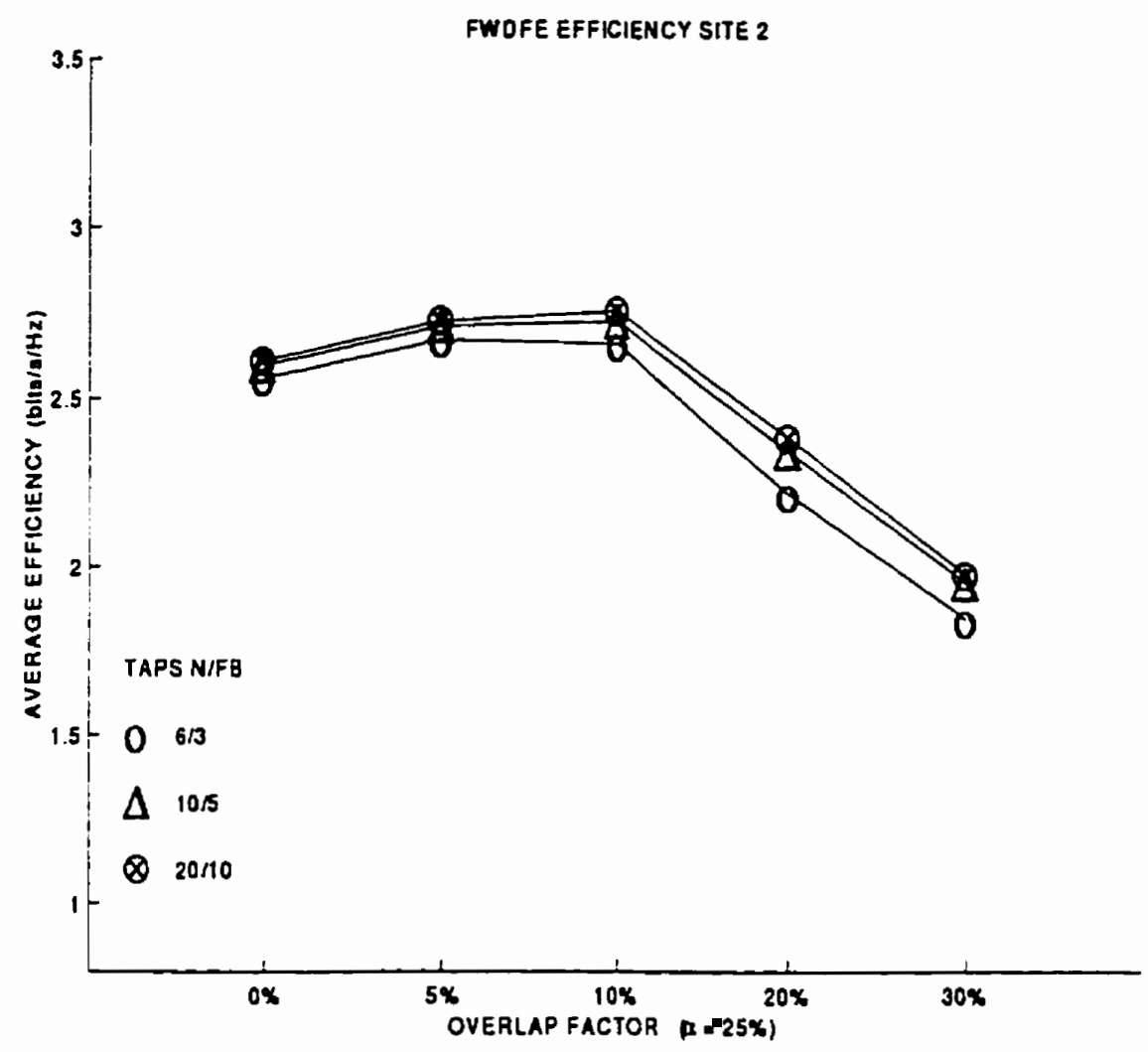

Figure 4.33: System efficiency for the high portion of the spectrum (15-35 $\mathrm{MHz}$ ) versus overlapping factor for an equivalent raised cosine filter with $25 \%$ excess bandwidth and 2.1 $\mathrm{MHz}$ sub-bands. 


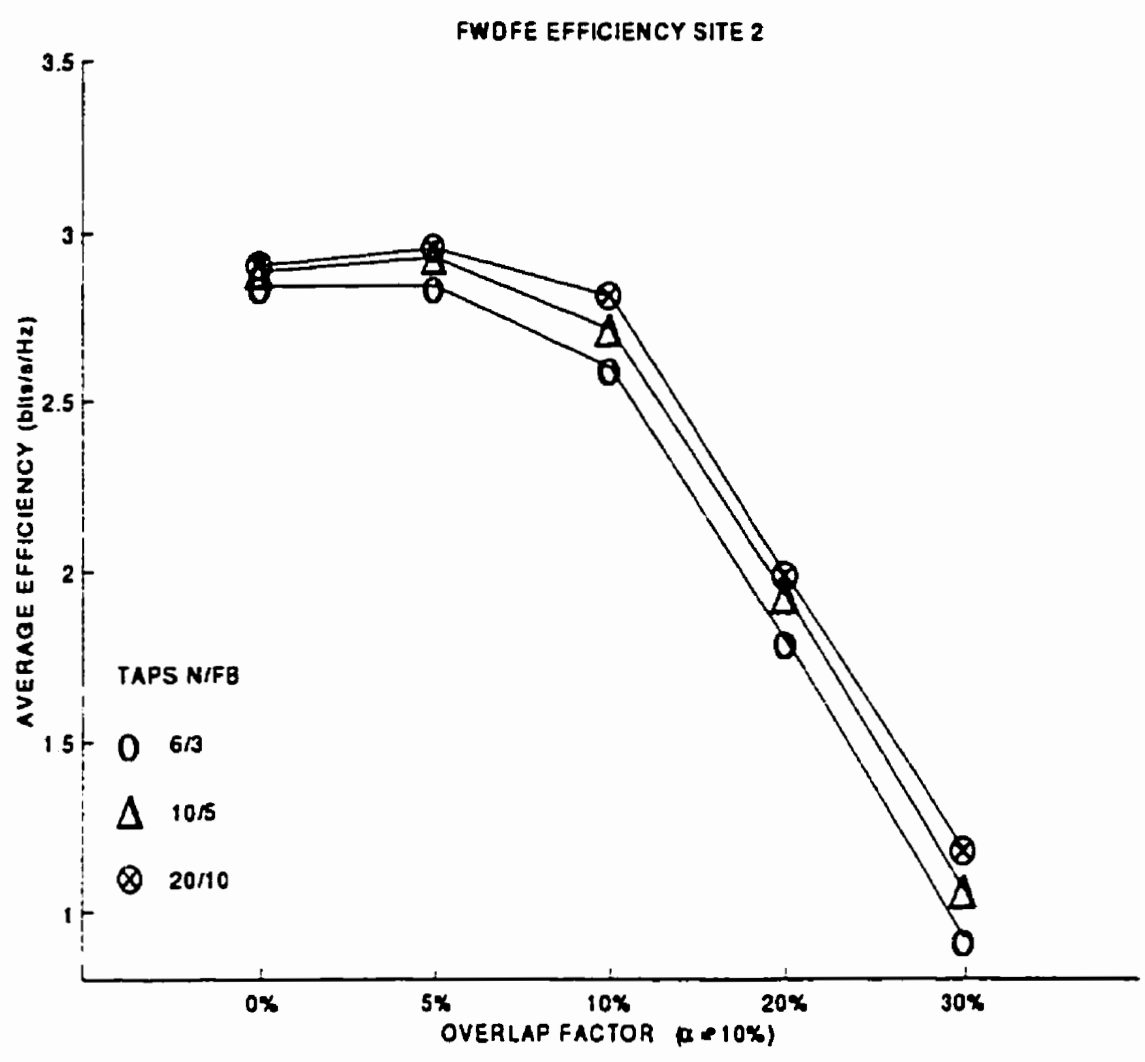

Figure 4.34: System efficiency for the high portion of the spectrum (15-35 $\mathrm{MHz}$ ) versus overlapping factor for an equivalent raised cosine filter with $10 \%$ excess bandwidth and $2.1 \mathrm{MHz}$ sub-bands. 


\subsubsection{Fractionally spaced equalizers}

This section is similar to the previous one except that we now will consider the effect of fractionally spaced equalization with a 20/10 taps equalizer to mitigate the adjacent channel interference and the channel distortion.

The graphs

Three curves are shown representing the fractional spacing of $T / 1(0)$, $T / 2(\Delta)$ and $T / 4(\otimes)$. Each curve plots the average efficiency versus the overlapping factor of $0 \%, 5 \%, 10 \%, 20 \%$ and $30 \%$. The letter ' $s$ ' represents the factor for the fractionally spacing used.

Figures $4.35,4.36,4.37$ and 4.38 present the results for the low portion and high portion of the spectrum with $25 \%$ and $10 \%$ excess bandwidth raised cosine filters. A significant improvement is observed when we use fractional spacing in all the figures. This can be explained by the fact that a synchronous equalizer cannot exercise independent control on both sides of the rolloff region (at $\mathrm{f}=1 / 2 \mathrm{~T}$ ). It will only act on the aliased spectrum instead of the real spectrum. If we use a fractionally spaced equalizer that samples at least twice the input sample frequency, however, the first repetition of the spectrum would include the rolloff region [25]. The best performance was obtained with the $\mathrm{T} / 4$ fractionally spaced equalizer. For the filter with $10 \%$ excess bandwidth, we obtained the best performance with an overlapping factor of $10 \%$ (carrier spacing of $1 / T$ ). For the filter with $25 \%$ excess bandwidth, we have better performance with an overlapping factor of $30 \%$ (carrier spacing of $1 / \mathrm{T}-5 \%$ ). 
An interesting duality appears between the fractional spacing of $T / 2$ and the synchronous equalizer $(\mathrm{T} / 1)$. If the overlap factor is large enough, the synchronous equalizer will have better performance. This can be explained by the fact that there are 3 parameters involved in these computations: the channel distortion; the excess bandwidth; and the fractional spacing. If the fractionally spaced equalizer cannot entirely remove the effect of channel distortion and adjacent channel interference, it is possible that the synchronous equalizer will give better performance since its taps are spread out over a longer period of time and can thus deal more effectively with the channel distortion. 


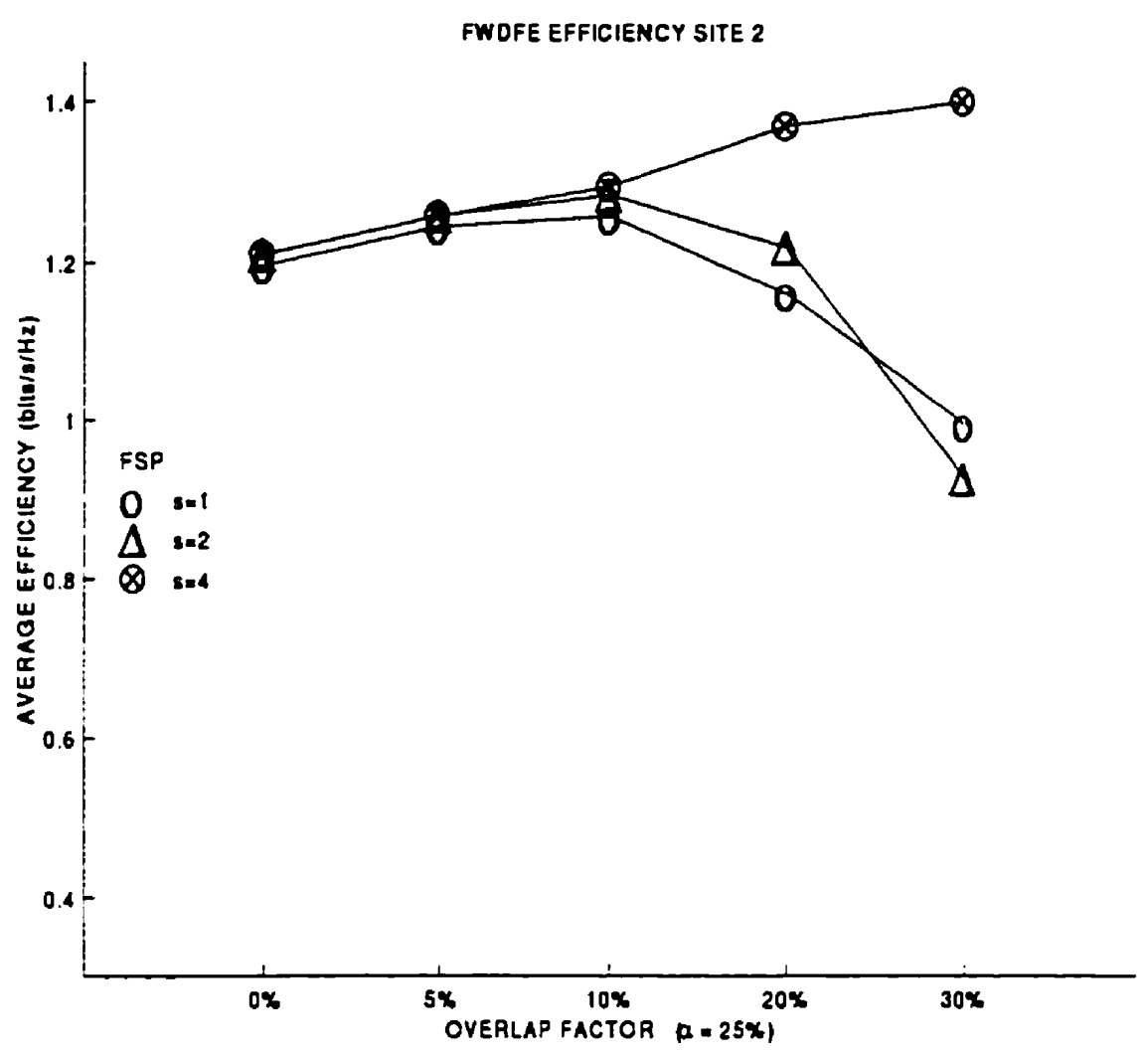

Figure 4.35: System efficiency for the low portion of the spectrum (5-15 MHz) versus overlapping factor for an equivalent raised cosine filter with $25 \%$ excess bandwidth and $2.1 \mathrm{MHz}$ sub-bands. 


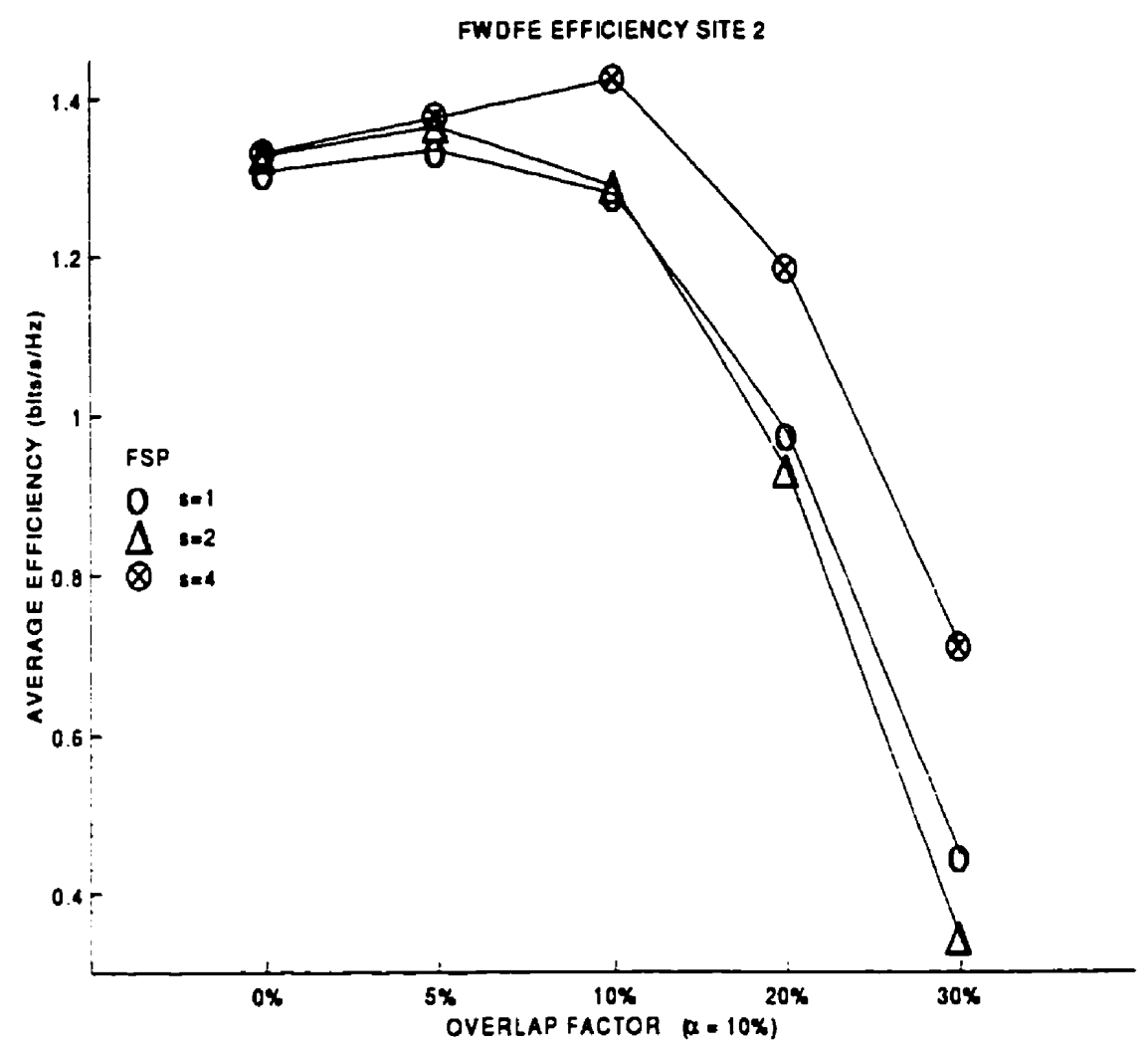

Figure 4.36: System efficiency for the low portion of the spectrum (5-15 MHz) versus overlapping factor for an equivalent raised cosine filter with $10 \%$ excess bandwidth and $2.1 \mathrm{MHz}$ sub-bands. 


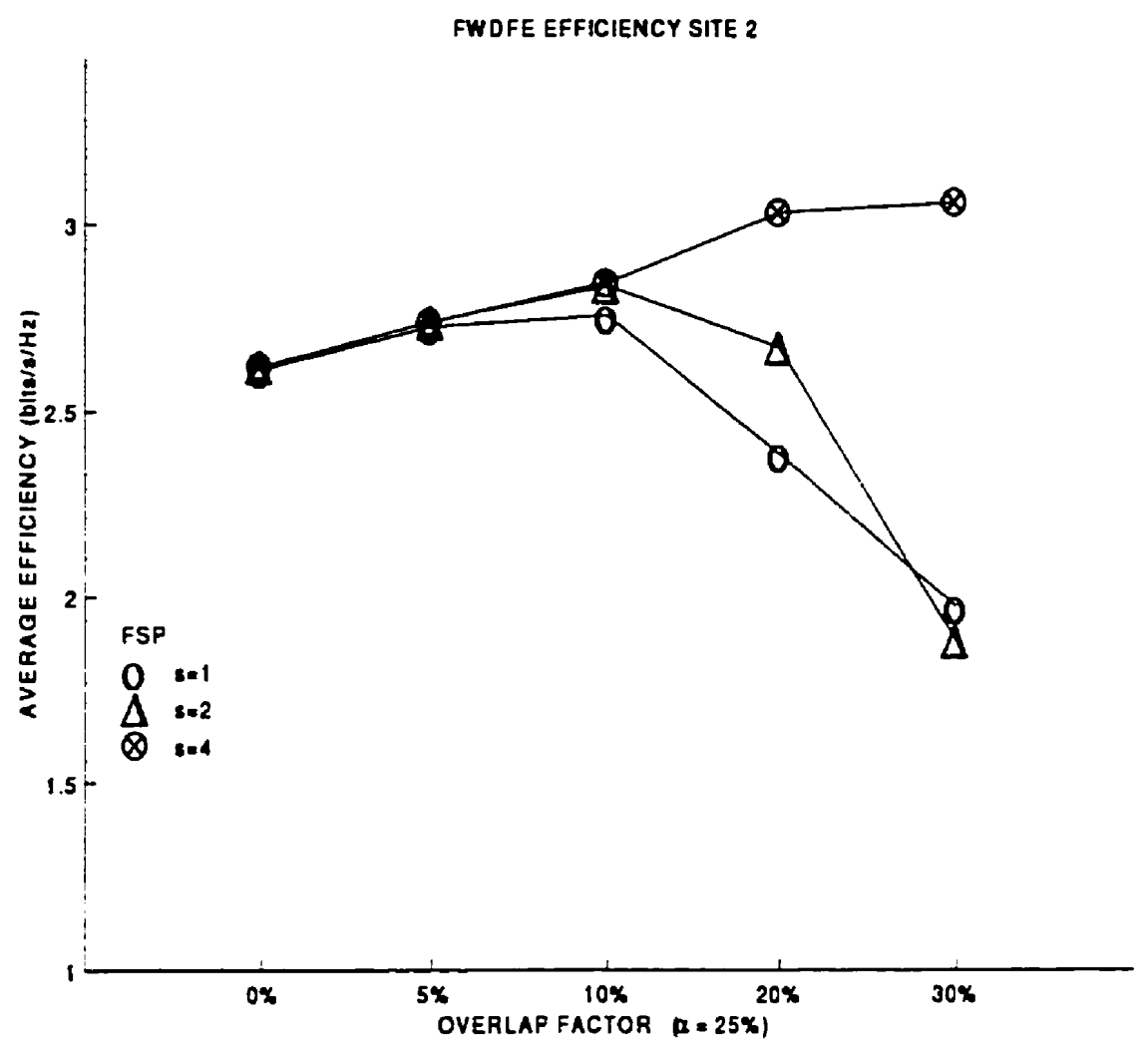

Figure 4.37: System efficiency for the high portion of the spectrum (15-35 $\mathrm{MHz}$ ) versus overlapping factor for an equivalent raised cosine filter with $25 \%$ excess bandwidth and $2.1 \mathrm{MHz}$ sub-bands. 


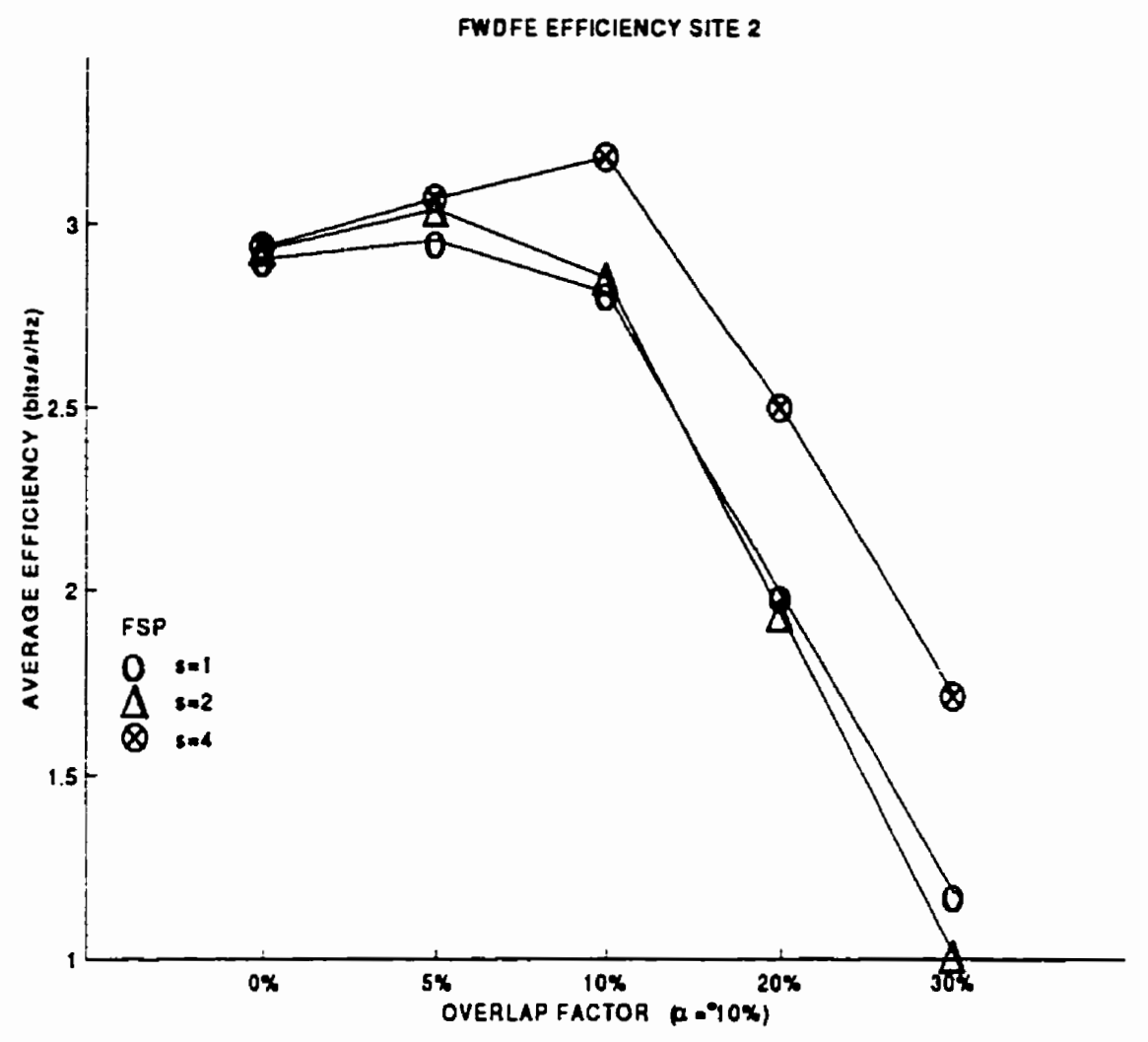

Figure 4.38: System efficiency for the high portion of the spectrum (15-35 $\mathrm{MHz}$ ) versus overlapping factor for an equivalent raised cosine filter with $10 \%$ excess bandwidth and $2.1 \mathrm{MHz}$ sub-bands. 


\subsubsection{Performance of WFDFE and other models}

The objective of this section is to compare the performance of the WFDFE, the WDFE and the MCM models. The WFDFE and the WDFE systems have been analyzed using sub-band widths of 2.1 and $10.1 \mathrm{MHz}$. The MCM system has been analyzed with sub-bands of $100 \mathrm{kHz}$. Results for both sites are also shown.

The models are defined as follows:

MCM: This is the ideal parallel modulation model as defined in section 3.2. It consists of $100 \mathrm{kHz}$ rectangular sub-bands. Channel distortion and guardbands are not included.

WDFE: This is the ideal serial modulation model as defined in section 3.3 with infinite numbers of DFE taps. We assume rectangular sub-bands with no overlapping spectra. No channel distortion is considered.

FDMA: This model is a generalization of the MCM model as it repeats the same analysis with wider sub-bands. In this case we considered channel distortion and assumed rectangular sub-bands with no overlapping spectra.

WFDFE: This is the model which presented the best performance in the presence of adjacent channel interference. The finite equalizer consists of a 20/10 (forward/feedback) taps fractionally spaced at T/4 DFE for the 2 $\mathrm{MHz}$ sub-band system and a $40 / 20$ (forward/feedback) taps fractionally 
spaced at $\mathrm{T} / 4 \mathrm{DFE}$ for the $10 \mathrm{MHz}$ sub-band system. We consider the effect of guardbands and the effect of the adjacent channel interference by having carriers spaced at $1 / \mathrm{T}-5 \%$ with an excess bandwidth parameter of $25 \%$.

For the models with channel distortion, we assumed a channel with 1 reflection of $500 \mathrm{nsec}$ and $-20 \mathrm{db}$ down.

Table 4.7 and 4.8 show the channel capacity for the models for the low and the high portion of the spectrum for site 1. Table 4.9 and 4.10 show the channel capacity for the models for the low and the high portion of the spectrum for site 2 . The FCA-1\% and VCA-1\% represent the capacity with $1 \%$ outage. The VCA-AVG represents the average capacity obtained with the VCA scheme as defined in section 3.2.4.

These tables provide a summary of the performance of each method and condenses the details discussed in previous sections.

Comparing the WFDFE and WDFE and the FDMA models:

It is interesting to see the gain in performance over the traditional FDMA scheme by comparing the WFDFE column with the FDMA column in all the tables. We see a slight degradation for the finite DFE in comparison with the infinite DFE. Note that the infinite DFE has infinite taps and a continuous sampling rate which explains the slight degradation for the finite case. Notice also that the WFDFE and the WDFE have almost the same performance for the $15-35 \mathrm{MHz}$ portion of the spectrum. 
Table 4.7: Channel capacity in Mbps for the low (5-15 MHz) portion of the spectrum for site 1 .

\begin{tabular}{|c|c|c|c|c|}
\hline Scheme, Outage, sub-band & MCM & WDFE & FDMA & WFDFE \\
\hline \hline FCA, 1\%,2 MHz & 0 & 0 & 0 & 0 \\
\hline VCA, 1\%, 2 MHz & 1.8 & 0 & 0 & 0 \\
\hline VCA-AVG, $2 \mathrm{MHz}$ & 15.6 & 14.1 & 7.8 & 10.2 \\
\hline FCA, 1\%, 10 MHz & 0 & 0 & 0 & 0 \\
\hline VCA, 1\%, 10 MHz & 1.8 & 0 & 0 & 0 \\
\hline VCA-AVG, $10 \mathrm{MHz}$ & 15.6 & 14.8 & 0 & 7.3 \\
\hline
\end{tabular}

Table 4.8: Channel capacity in Mbps for the high (15-35 MHz) portion of the spectrum for site 1 .

\begin{tabular}{|c|c|c|c|c|}
\hline Scheme, Outage, sub-band & MCM & WDFE & FDMA & WFDFE \\
\hline \hline FCA, $1 \%, 2 \mathrm{MHz}$ & 25 & 34 & 14 & 23 \\
\hline VCA, $1 \%, 2 \mathrm{MHz}$ & 42.8 & 42 & 24 & 27.2 \\
\hline VCA-AVG, $2 \mathrm{MHz}$ & 68.2 & 70.4 & 52.8 & 53.6 \\
\hline FCA, $1 \%, 10 \mathrm{MHz}$ & 25 & 40 & 20 & 30.8 \\
\hline VCA, $1 \%, 10 \mathrm{MHz}$ & 42.8 & 40 & 30 & 41 \\
\hline VCA-AVG, $10 \mathrm{MHz}$ & 68.2 & 71.2 & 63.6 & 65.6 \\
\hline
\end{tabular}


Table 4.9: Channel capacity in $\mathrm{Mbps}$ for the low (5-15 MHz) portion of the spectrum for site 2 .

\begin{tabular}{|c|c|c|c|c|}
\hline Scheme, Outage, sub-band & MCM & WDFE & FDMA & WFDFE \\
\hline \hline FCA, $1 \%, 2 \mathrm{MHz}$ & 3.2 & 4 & 0 & 2.1 \\
\hline VCA, $1 \%, 2 \mathrm{MHz}$ & 9.6 & 8 & 2 & 4.2 \\
\hline VCA-AVG, $2 \mathrm{MHz}$ & 19.7 & 17.4 & 11 & 14 \\
\hline FCA, 1\%, 10 MHz & 3.2 & 10 & 0 & 0 \\
\hline VCA, 1\%, 10 MHz & 9.6 & 10 & 0 & 0 \\
\hline VCA-AVG, $10 \mathrm{MHz}$ & 19.7 & 17.2 & 10 & 10.8 \\
\hline
\end{tabular}

Table 4.10: Channel capacity in Mbps for the high (15-35 MHz) portion of the spectrum for site 2 .

\begin{tabular}{|c|c|c|c|c|}
\hline Scheme, Outage, sub-band & MCM & WDFE & FDMA & WFDFE \\
\hline \hline FCA, $1 \%, 2 \mathrm{MHz}$ & 34.2 & 42 & 20 & 29.4 \\
\hline VCA, $1 \%, 2 \mathrm{MHz}$ & 41.2 & 42 & 24 & 33.6 \\
\hline VCA-AVG, $2 \mathrm{MHz}$ & 70.8 & 73.4 & 59.2 & 61.2 \\
\hline FCA, 1\%, 10 MHz & 34.2 & 40 & 10 & 41 \\
\hline VCA, $1 \%, 10 \mathrm{MHz}$ & 41.2 & 40 & 20 & 41 \\
\hline VCA-AVG, $10 \mathrm{MHz}$ & 70.8 & 75.4 & 67.2 & 72.2 \\
\hline
\end{tabular}




\section{Highlights:}

- WDFE shows almost the same performance when either the VCA- $1 \%$ or FCA-1\% schemes are used in the $15-35 \mathrm{MHz}$ portion of the spectrum. This occurred in both sites and for both sub-band widths. This can be explained by the fact that the WDFE is able to mitigate the ingress noise present in the upstream channel (refer to section 4.3.2).

- The usage of smaller bandwidth is only relevant in the lower portion of the spectrum for a wideband system. This can be explained by the fact that the WDFE cannot entirely mitigate the ingress noise and the effect of using smaller-bandwidth starts to prevail (refer to section 4.3.2). An MCM system could have an advantage in that region by using smaller sub-band width.

- In the high portion of the spectrum, the WDFE has slightly better performance over the MCM scheme with the VCA-AVG capacity scheme for both sites and for both sub-band widths. As was mentioned in section 4.4, prior research has shown that MCM and a wideband system with DFE presents similar results for high SNR.

- For the MCM method, the VCA scheme greatly improves the performance in comparison to a FCA scheme. The VCA scheme is able to adapt the modulation scheme to the time varying aspect of the noise (refer to section 4.2.2). 
- The first $10 \mathrm{MHz}$ of the upstream path is severely hampered by ingress noise. This is more evident in site 1 where only very low bit rate can be obtained with the usage of MCM.

\section{WFDFE performance:}

We obtained good performance for the WFDFE with $10 \mathrm{MHz}$ sub-bands for the 15-35 MHz portion of the spectrum for site 2. The $40 / 20$ (forward/feedback) taps equalizer with $1 / \mathrm{t}-5 \%$ carrier spacing is able to mitigate the adjacent channel interference and the ingress noise present in that portion of the spectrum. This result is of less importance for the $2 \mathrm{MHz}$ sub-bands case. This could be caused by the frequency resolution of the spectrum analyzer which affects the evaluation of the WFDFE. Only 20 points can be used in this case to compute the taps coefficients in comparison to 100 points for $10 \mathrm{MHz}$ sub-band widths. By having the resulting spectrum diverging from reality and fewer points to perform the evaluation, the $2 \mathrm{MHz}$ sub-band width case could differ slightly from the optimum results. Nevertheless, the WFDFE - FCA seems to be a viable alternative to a complex scheme such as MCM - VCA. 


\section{Chapter 5}

\section{Conclusions and}

\section{recommendations}

The results have shown that both the parallel transmission of data and the serial transmission of data would result in roughly similar performance for the $15-35 \mathrm{MHz}$ portion of the upstream path when used with a VCA scheme. The time variability of the noise is an important aspect in the upstream path, and special care should be taken especially in the first 5-15 MHZ portion. The usage of small sub-bands combined with a VCA scheme is recommended in order to use this portion effectively. Otherwise, efficient forward error coding will need to be implemented.

We have seen that the MCM system is effective in mitigating the ingress noise found in the upstream path. It is recommended to use an adaptive and variable channel allocation scheme to obtain good performance with the MCM 
method in comparison to a wideband system with DFE.

In situations where a system of lower complexity would be considered, a wideband system with a DFE using an FCA scheme is a good solution. The WDFE-FCA has better performance than that of a MCM-FCA scheme and would be simpler to implement by using fixed bit rate allocation for individual sub-bands instead of a more complex adaptive data allocation method. A reasonable number of taps, such as $40 / 20$ (forward/feedback) for a $10 \mathrm{MHz}$ system and $20 / 10$ for a $10 \mathrm{MHz}$ system, have proven to be efficient for the decision feedback equalizer. The DFE equalizer as proven to be effective in mitigating the ingress noise and reflections found in the upstream path and was also proven to be effective against adjacent channel interference. It was shown that we can improve the upstream capacity by using fractionally spaced equalization and carriers spaced in such way that their individual spectra overlap.

\subsection{Future research}

In regards to our approach, further research should be done using measured ingress spectra with better resolution. Research should also be done on the effect of impulse noise for the MCM system and the wideband DFE system. It was mentioned that both systems would behave differently towards impulse noise. A MCM system would be more sensitive to tones in the frequency domain while a wideband system might be more sensitive to impulse in the 
time domain. The effect of phase jitter and symbol synchronization should also be analyzed for the MCM system. Improper symbol synchronization can affect the orthogonality between symbols in MCM systems and phase jitter could cause spectral leaks that would be added to the noise on a power basis.

Other areas of research include the interaction between the modulation method and the medium access control protocols, and the effect of forward error control coding methods versus upstream channel performance considering various portions of the spectrum. 


\section{Bibliography}

[1] D. Large, "Creating a Network for Interactivity", IEEE Spectrum, April 1995: pp 58-63.

[2] E. Perry and S. Ramanathan, "Network Management for Residential Broadband Interactive Data Services", IEEE Communications Magazine, November 1996: pp 114-121.

[3] C. Bisdikan, K. Maruyama, D. I. Seidman and D. N. Serpanos, "Cable Access Beyond the Hype. On Residential Broadband Data Services over HFC Networks", IEEE Communications Magazine, November 1996: pp 128-135.

[4] G. A. Hart and R. Lyford, "Deploying Telephony Services Over CATV Systems: Systems and Architectural Considerations", NCTA Technical Papers, 1994: pp 206-218.

[5] J.E. Dail, M. A. Dajer, C. Li et al., "Adaptive Digital Access Protocol: A MAC Protocol for Multiservice Broadband Access Networks", IEEE 
Communications Magazine, March 1996: pp 104-112.

[6] C. Bisdikian, B. Mcneil, R. Norman and Ray Zeisz, "MLAP: A MAC Level Access Protocol for the HFC 802.14 Network", IEEE Communications Magazine, March 1996: pp 114-120.

[7] R. S. Prodan, M. Chelehmal, T. H. Williams and C. M. Chamberlain, "Analysis of Cable System Digital Transmission Characteristics", NCTA Technical Papers, 1994: pp 254-258.

[8] R. K. Jurgen, "Broadcasting with Digital Radio", IEEE Spectrum, March 1996: pp 52-59.

[9] D. C. Coll, "Cable Television", The Froehlich/Kent Encyclopedia of Telecommunications, volume 2, Marcel Dekker, 1991.

[10] K. S. Jacobsen and J. M. Cioffi, "High-performance Multimedia Transmission on the Cable Television Network", Proc. Int. Conference on Communications, 1994: pp 1048-1052.

[11] J. Barselloti and B. Langlais, "Overcoming Problems in the CATV Return Path with OFDM", Conference on Emerging Technologies, January 1996: pp 319-328.

[12j J. Bingham, "Multicarrier Modulation for Data Transmission: An Idea Whose Time Has Come", IEEE Communications Magazine, May 1990: pp 5-14. 
[13] B R. Petersen and D. Falconer, "Suppression of Adjacent-Channel, Cochannel, and Intersymbol Interference by Equalizers and Linear Combiners", IEEE Transaction on Communications, December 1994: pp 31093118 .

[14] B. Sklar, Digital Communications Fundamentals and Applications, Prentice Hall, 1988.

[15] J. Proakis, Digital Communication, McGraw-Hill, 1995: pp 621-625.

[16] N. Zervos and I. Kalet, "Optimized Decision Feedback Equalizer Versus Optimized Orthogonal Frequency Division Multiplexing for High-Speed Data Transmission Over the Local Cable Network", Proc. Int. Conference on Communications, September 1989: pp 1080-1085.

[17] C. Eldering, M. Kauffman, D. Grubb et al., "Engineering Requirements for Hybrid Fiber-Coax Telephony Systems", NCTA Technical Papers, 1994: pp 219-231.

[18] A. J. Kim, "Two-Way Plant Characterization", Network Integrity Working Group, 1995.

[19] D. Falconer, "Application of Passband Decision Feedback Equalization in Two- Dimensional Data Communication Systems", IEEE Transaction on Communications, October 1976: pp 1159-1166. 
[20] A. L. Garcia, Probability and Random Processes for Electrical Engineering, Addison Wesley, May 1994.

[21] S. Qureshi, "Adaptive Equalization", IEEE Communications Magazine, March 1982: pp 9-16.

[22] J. M. Cioff, G. P. Dudevoir, M. V. Eyuboglu and G. D. Forney, "MMSE Decision-Feedback Equalizers and Coding-Part 1", IEEE Transaction on Communications, October 1995: pp 2582-2594.

[23] J. M. Cioff, G. P. Dudevoir, M. V. Eyuboglu and G. D. Forney, "MMSE Decision-Feedback Equalizers and Coding-Part 2", IEEE Transaction on Communications, October 1995: pp 2595-2604.

[24] G. Cherubini, J. Creigh, S. Olcer, S. K. Rao and G. Ungerboeck, "100BASE-T2: $100 \mathrm{Mbit} / \mathrm{s}$ Ethernet over Two Pairs of Category-3 Cabling", Proc. Int. Conference on Communications, June 1997: pp 10141018.

[25] R. D. Gitlin, J. F. Hayes and S. B. Weinstein, Data Communication Principle, Plenum Press, 1992: pp 493-496. 
IMAGE EVALUATION

TEST TARGET (QA-3)
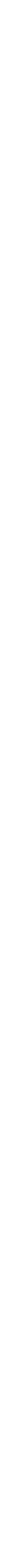